\title{
Rhodium(III)-Catalyzed C-H Olefination of Aromatic/Vinyl Acids with Unactivated Olefins at Room Temperature
}

\author{
Subramanian Jambu and Masilamani Jeganmohan*
}

Department of Chemistry, Indian Institute of Technology Madras, Chennai 600036,

Tamil Nadu, India

Email:mjeganmohan@iitm.ac.in

\section{Supporting Information (SI)}

\section{Table of Contents}

$\begin{array}{ll}\text { S2 - S3 } & \text { Experimental Section } \\ \text { S4 - S8 } & \text { Intermediate isolation and Mechanistic Studies (Deuterium labelling) } \\ \text { S9-S10 } & \text { Optimization Studies } \\ \text { S11 - S12 } & \text { X-Ray analysis } \\ \text { S13 } & \text { References } \\ \text { S14-S35 } & \text { Spectral Data of all Compounds } \\ \text { S36 - S77 } & \text { Copies of }{ }^{1} \mathrm{H} \text { and }{ }^{13} \text { C NMR Spectra of All Compounds. }\end{array}$




\section{Experimental Section}

General Information. All reactions were carried out under the $\mathrm{N}_{2}$ atmosphere in flame-dried glassware. Syringes which were used to transfer anhydrous solvents or reagents were purged with nitrogen prior to use (three times). Dry solvents were used for the reaction. Column chromatographical purifications were performed using $\mathrm{SiO}_{2}$ (100-200 mesh ASTM) from Avra Pvt. Ltd., India. Abbreviations for signal coupling are as follows: s, singlet; d, doublet; t, triplet; q, quartet; m, multiplet. $\left[\mathrm{Rh}\left(\mathrm{Cp}^{*}\right) \mathrm{Cl}_{2}\right],{ }^{1}$ were prepared according to literature procedures. Commercially available Aromatic Acids, Alkenes, metal salts and other chemicals were purchased from Sigma-Aldrich and Spectrochem. Pvt. Ltd., India. $\mathrm{Na}_{2} \mathrm{HPO}_{4}$ was purchased from Thermo Fisher Scientific Private Limited, India. These chemicals were used without further purification.

\section{General Procedure for the Vinylation/Branched/Allylation Reaction.}

Aromatic Acids 1 (50 mg) (1 equiv), [ $\left.\mathrm{Rh}\left(\mathrm{Cp}^{*}\right) \mathrm{Cl}_{2}\right],(5 \mathrm{~mol} \%), \mathrm{Na}_{2} \mathrm{HPO}_{4}\left(1\right.$ equiv) and $\mathrm{Ag}_{2} \mathrm{O}$ $(50 \mathrm{~mol} \%)$ were taken in a $15 \mathrm{~mL}$ pressure tube. $N, N$-dimethylformamide (DMF) $(2.0 \mathrm{~mL})$ was added to the reaction mixture. Then, alkenes 2 ( 4 equiv) were added followed by the addition of DMF (1.0 mL). The reaction mixture was evacuated and purged with nitrogen gas three times. Then the septum was taken out and immediately screw cap was used to cover the tube. The reaction mixture was allowed to stir at $\mathrm{rt}$ for $24 \mathrm{~h}$. After the reaction was complete, it was diluted with $\mathrm{CH}_{2} \mathrm{Cl}_{2}$, filtered through celite, and the filtrate was concentrated. Then, $1 \mathrm{~N}$ $\mathrm{HCl}(15 \mathrm{~mL})$ was added to the reaction mixture and extracted with EtOAc $(1 \mathrm{x} 15 \mathrm{~mL})$. The organic layer was washed with cold water $(2 \times 15 \mathrm{~mL})$ followed by brine workup $(10 \mathrm{~mL})$ and dried using $\mathrm{Na}_{2} \mathrm{SO}_{4}$. The organic layer was filtered and concentrated under reduced pressure. The crude residue was purified through column chromatography using $\mathrm{SiO}_{2}$, hexane, ethyl acetate and $1 \% \mathrm{HCOOH}$ as eluent to give pure product 3 . In the case of functionalized unactivated alkenes $\mathbf{2} \mathbf{j}-\mathbf{m}, 2.0$ equiv was used.

\section{Procedure for the vinylation of 2-Methoxy benzoic acid (1a) with Vinyl Cyclohexane} (2a) Catalyzed by a Rhodium Complex (1 mmol scale).

2-Methoxy benzoic acid 1a (153 mg, $1 \mathrm{mmol}),\left[\mathrm{Rh}\left(\mathrm{Cp}^{*}\right) \mathrm{Cl}_{2}\right],(5 \mathrm{~mol} \%, 0.05 \mathrm{mmol}, 31 \mathrm{mg})$, $\mathrm{Na}_{2} \mathrm{HPO}_{4}$ (1 equiv, $1 \mathrm{mmol}, 142 \mathrm{mg}$ ) and $\mathrm{Ag}_{2} \mathrm{O}$ (50 mol \%, $0.5 \mathrm{mmol}, 116 \mathrm{mg}$ ) were taken in a $15 \mathrm{~mL}$ pressure tube. $N, N$-dimethylformamide (DMF) $(3.0 \mathrm{~mL})$ was added to the reaction mixture. Then, vinylcyclohexane 2a (4 equiv, $4 \mathrm{mmol}, 0.55 \mathrm{~mL}$ ) was added followed by the addition of DMF (2.0 mL). The reaction mixture was evacuated and purged with nitrogen gas three times. Then the septum was taken out and immediately screw cap was used to cover the 
tube. The reaction mixture was allowed to stir at $\mathrm{rt}$ for $24 \mathrm{~h}$. After the reaction was complete, it was diluted with $\mathrm{CH}_{2} \mathrm{Cl}_{2}$, filtered through celite, and the filtrate was concentrated. Then, $1 \mathrm{~N}$ $\mathrm{HCl}(30 \mathrm{~mL})$ was added to the reaction mixture and extracted with EtOAc $(1 \mathrm{x} 30 \mathrm{~mL})$. The organic layer was washed with cold water $(2 x 30 \mathrm{~mL})$ followed by brine workup $(20 \mathrm{~mL})$ and dried using $\mathrm{Na}_{2} \mathrm{SO}_{4}$. The organic layer was filtered and concentrated under reduced pressure. The crude residue was purified through column chromatography using $\mathrm{SiO}_{2}$, hexane, ethyl acetate and $1 \% \mathrm{HCOOH}$ as eluent to give pure product 3aa $(190 \mathrm{mg})$ in $74 \%$ yield as a white solid.

\section{Procedure for the Competitive Experiment between $1 \mathrm{j}$ and 1k with $2 \mathrm{a}$.}

An aromatic acid $\mathbf{1 j}(0.25 \mathrm{mmol}), \mathbf{1 k}(0.25 \mathrm{mmol}),\left[\mathrm{Rh}\left(\mathrm{Cp}^{*}\right) \mathrm{Cl}_{2}\right],(5 \mathrm{~mol} \%), \mathrm{Na}_{2} \mathrm{HPO}_{4}(1$ equiv) and $\mathrm{Ag}_{2} \mathrm{O}(50 \mathrm{~mol} \%)$ were taken in a $15 \mathrm{~mL}$ pressure tube. $N, N$-Dimethylformamide (DMF) $(2.0 \mathrm{~mL}$ ) was added to the reaction mixture. Then, an alkene $2 \mathbf{a}$ (4.0 equiv) were added followed by the addition of DMF $(1.0 \mathrm{~mL})$. The reaction mixture was evacuated and purged with nitrogen gas three times. Then the septum was taken out and immediately screw cap was used to cover the tube. The reaction mixture was allowed to stir at $\mathrm{rt}$ for $24 \mathrm{~h}$. After the reaction was complete, it was diluted with $\mathrm{CH}_{2} \mathrm{Cl}_{2}$, filtered through celite, and the filtrate was concentrated. Then, $1 \mathrm{~N} \mathrm{HCl}(15 \mathrm{~mL})$ was added to the reaction mixture and extracted with EtOAc ( 1 x $15 \mathrm{~mL})$. The organic layer was washed with cold water $(2 \times 15 \mathrm{~mL})$, brine workup $(10 \mathrm{~mL})$ and dried using $\mathrm{Na}_{2} \mathrm{SO}_{4}$, filtered and concentrated under reduced pressure. The crude residue was purified through column chromatography using $\mathrm{SiO}_{2}$, hexane, ethyl acetate and $1 \% \mathrm{HCOOH}$ as eluent to give pure product $3 \mathbf{j} \mathbf{j a}$ and $3 \mathbf{k a}$. In the reaction, product $\mathbf{3 j a}$ was observed in 33\% yield and 3ka in 30\% yield, respectively.

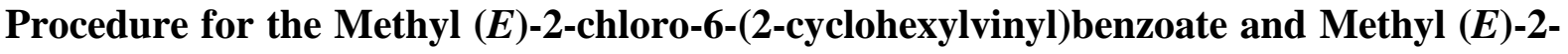
bromo-6-(2-cyclohexylvinyl)benzoate.

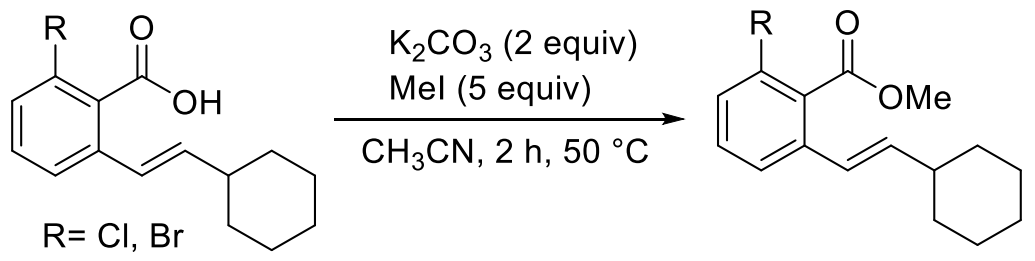

A $15 \mathrm{~mL}$ pressure tube with a septum containing (E)-2-chloro-6-(2-cyclohexylvinyl)benzoic acid (3da) (50 mg) or (E)-2-bromo-6-(2-cyclohexylvinyl)benzoic acid (3ea) was dissolved in acetonitrile (3.0 mL) solvent. Then, $\mathrm{K}_{2} \mathrm{CO}_{3}$ (2.0 equiv) and methyl iodide (5.0 equiv) were 
added and the mixture was stirred at $50^{\circ} \mathrm{C}$ for $2 \mathrm{~h}$. The completion of reaction was monitored by TLC. Water $(6 \mathrm{~mL})$ was added to the mixture and extracted with EtOAc $(3 \mathrm{x} 15 \mathrm{~mL})$. The combined organic layer was washed with brine $(2 \times 5 \mathrm{~mL})$, dried using $\mathrm{Na}_{2} \mathrm{SO}_{4}$, filtered, and concentrated under reduced pressure. The crude residue was purified by column chromatography silica-gel 120-200 mesh, 2\% EtOAc: Hexane gave corresponding ester products.

\section{Intermediate Isolation}

\section{Preparation and Characterization of Rhodacycle compound 7a.}

An oven-dried $25 \mathrm{~mL}$ Schlenk tube equipped with magnetic stirring bar was sequentially charged with $\mathbf{1 j}$ ( $0.25 \mathrm{mmol}, 25 \mathrm{mg}),\left[\mathrm{Cp}^{*} \mathrm{RhCl}_{2}\right]_{2}$ (0.5 equiv, $\left.50 \mathrm{mg}\right), \mathrm{KHCO}_{3}(0.5 \mathrm{mmol}, 33$ $\mathrm{mg})$, DMSO $(0.5 \mathrm{mmol}, 23 \mathrm{uL})$ and methanol $(5 \mathrm{~mL})$ in the air. The reaction mixture was stirred at $60{ }^{\circ} \mathrm{C}$ in oil bath for $1 \mathrm{~h}$. The solvent was evaporated and the crude was dissolved in dichloromethane $(5 \mathrm{~mL})$. The rhodacycle 7a was washed by stirring over $\mathrm{NaHCO}_{3}$ for $10 \mathrm{~min}$, and the phases were then separated. The organic phase was dried over anhydrous $\mathrm{Na}_{2} \mathrm{SO}_{4}$. The solvent evaporated and the rhodacycle 7a was collected as orange yellow soild (52\%, $40 \mathrm{mg})$. The structure of the product was confirmed by NMR and HRMS characterization. ${ }^{2}$

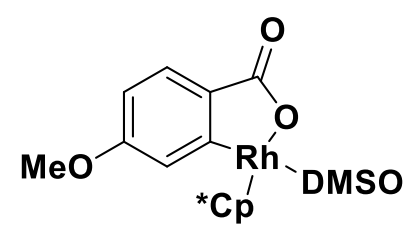

Chemical formula of complex: $\left(\left[\mathrm{C}_{20} \mathrm{H}_{27} \mathrm{O}_{4} \mathrm{RhS}\right] \mathrm{Na}\right)(\mathrm{M}+\mathrm{Na})$

HRMS (ESI-TOF) m/z: [M + Na $]^{+}$Calcd for $\mathrm{C}_{20} \mathrm{H}_{27} \mathrm{O}_{4} \mathrm{RhSNa} 489.0583$; Found 489.0570. 
${ }^{1} \mathrm{H}$ and ${ }^{13} \mathrm{C}$ NMR Spectra of Compound $7 \mathbf{a}\left(\mathrm{CDCl}_{3}\right.$ was used $)$.
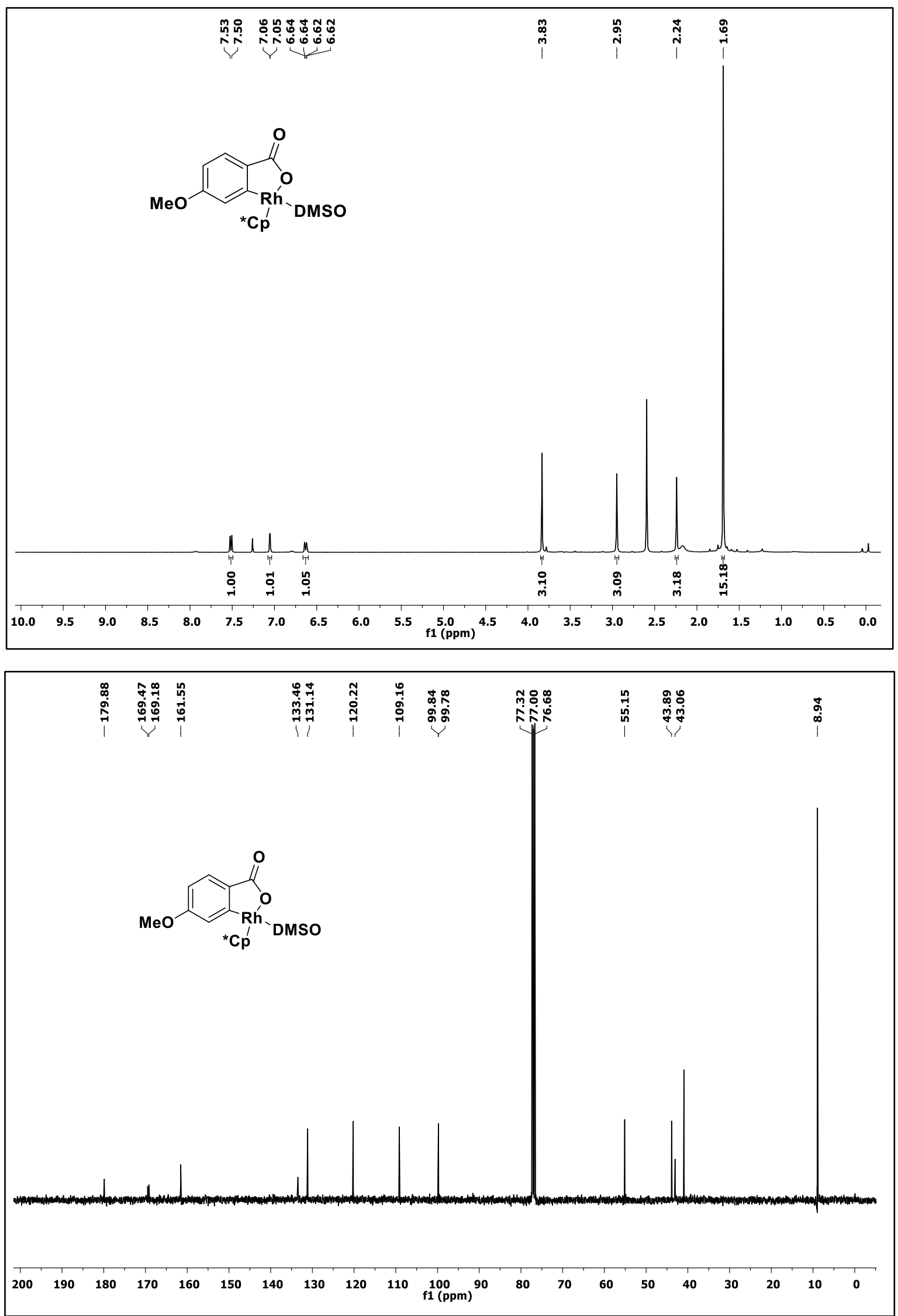


\section{Mechanistic Investigation}

\section{Deuterium Labelling Studies for D-1j.}

An aromatic acid $\mathbf{1 j}$ (50 mg) (1 equiv), $\left[\mathrm{Rh}\left(\mathrm{Cp}^{*}\right) \mathrm{Cl}_{2}\right],(5 \mathrm{~mol} \%), \mathrm{Na}_{2} \mathrm{HPO}_{4}\left(1\right.$ equiv) and $\mathrm{Ag}_{2} \mathrm{O}$ (50 mol \%) were taken in a $15 \mathrm{~mL}$ pressure tube. Then, $N, N$-dimethylformamide (DMF) (2.0 $\mathrm{mL}$ ) was added to the reaction mixture. Then, $\mathrm{CD}_{3} \mathrm{COOD}$ (2.0 equiv.) was added followed by the addition of DMF (1.0 mL) and again the reaction mixture was evacuated and purged with nitrogen gas three times. Then the septum was taken out and immediately screw cap was used to cover the tube. The reaction mixture was allowed to stir at $\mathrm{rt}$ for $12 \mathrm{~h}$. After the reaction was complete, it was diluted with $\mathrm{CH}_{2} \mathrm{Cl}_{2}$, filtered through Celite, and the filtrate was concentrated. Then, the reaction mixture was washed with ice water $(2 \times 15 \mathrm{~mL})$, brine $(10 \mathrm{~mL})$ and dried using $\mathrm{Na}_{2} \mathrm{SO}_{4}$, filtered and concentrated under reduced pressure. The crude residue was purified through column chromatography using $\mathrm{SiO}_{2}$, petroleum ether and ethyl acetates eluent to give pure product D-1j. In the reaction, product $\mathbf{D - 1 \mathbf { j }}$ was observed in $96 \%$ yield with $37 \%$ of deuterium incorporation at the both ortho carbons of 4-Methoxybenzoic acid, respectively. This result clearly reveals that the $\mathrm{C}-\mathrm{H}$ bond activation as a key intermediate in the reaction as well as it is the reversible process.

\section{Preparation of Compound D-3ja.}

An aromatic acid $\mathbf{1 j}$ (50 mg) (1 equiv), [Rh(Cp*)Cl $\mathrm{Cl}_{2}$, (5 mol \%), $\mathrm{Na}_{2} \mathrm{HPO}_{4}\left(1\right.$ equiv) and $\mathrm{Ag}_{2} \mathrm{O}$ $(50 \mathrm{~mol} \%)$ were taken in a $15 \mathrm{~mL}$ pressure tube. $N, N$-Dimethylformamide (DMF) $(2.0 \mathrm{~mL})$ was added to the reaction mixture. Then, an alkene $\mathbf{2 a}$ (4 equiv) and $\mathrm{CD}_{3} \mathrm{COOD}$ (2.0 equiv.) was added followed by the addition of DMF $(1.0 \mathrm{~mL})$. The reaction mixture was evacuated and purged with nitrogen gas three times. Then the septum was taken out and immediately screw cap was used to cover the tube. The reaction mixture was allowed to stir at $\mathrm{rt}$ for $24 \mathrm{~h}$. After the reaction was complete, it was diluted with $\mathrm{CH}_{2} \mathrm{Cl}_{2}$, filtered through celite, and the filtrate was concentrated. Then, the reaction mixture was washed with cold water $(2 x 15 \mathrm{~mL})$, brine workup $(10 \mathrm{~mL})$ and dried using $\mathrm{Na}_{2} \mathrm{SO}_{4}$, filtered and concentrated under reduced pressure. The crude residue was purified through column chromatography using $\mathrm{SiO}_{2}$, hexane, ethyl acetate and $1 \% \mathrm{HCOOH}$ as eluent to give pure product $\mathbf{D - 3 j a}$. In the reaction, product $\mathbf{D}-\mathbf{3 j a}$ was observed in $32 \%$ yield with $20 \%$ of deuterium incorporation at the ortho carbons of 4-Methoxybenzoic acid. 


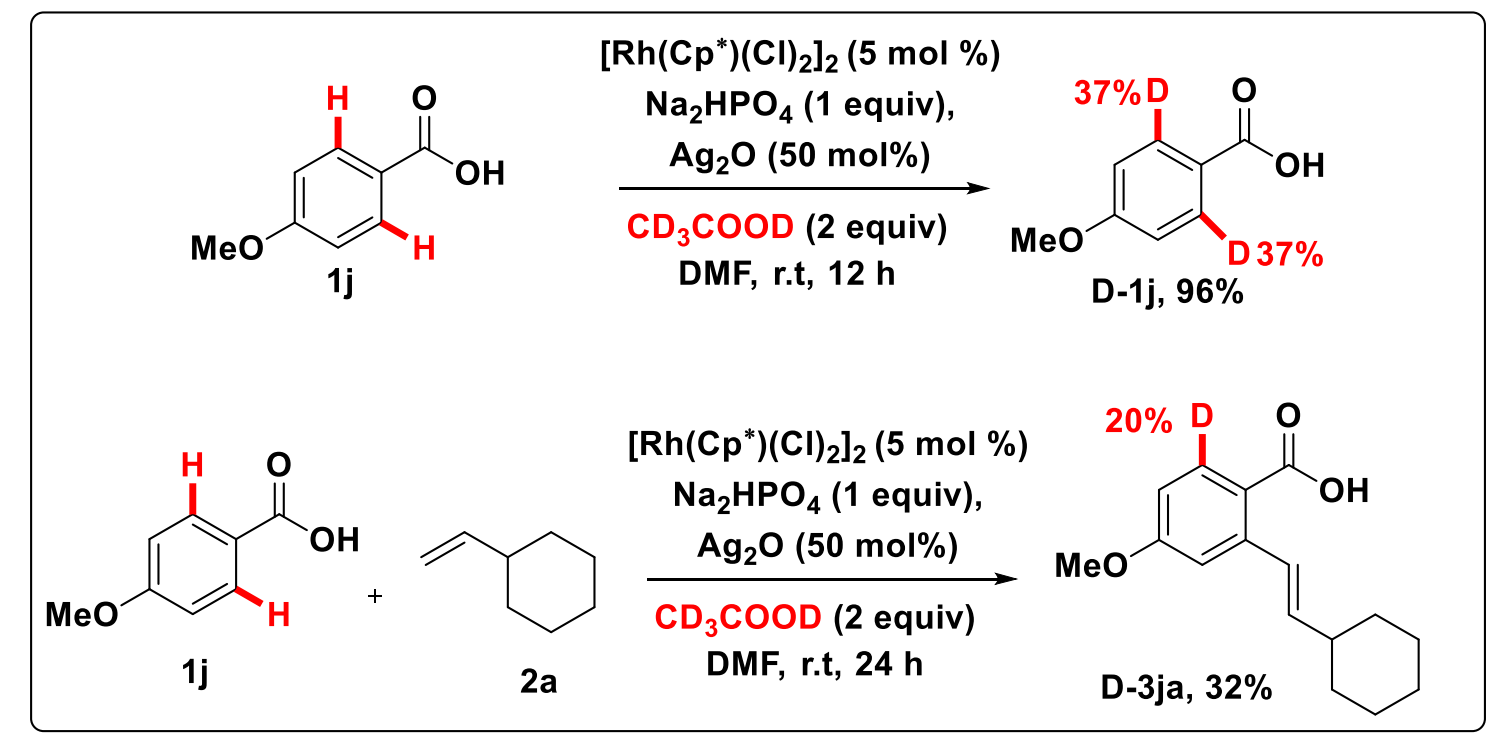


${ }^{1} \mathrm{H}$ Spectra of Compound D-1j (DMSO was used).

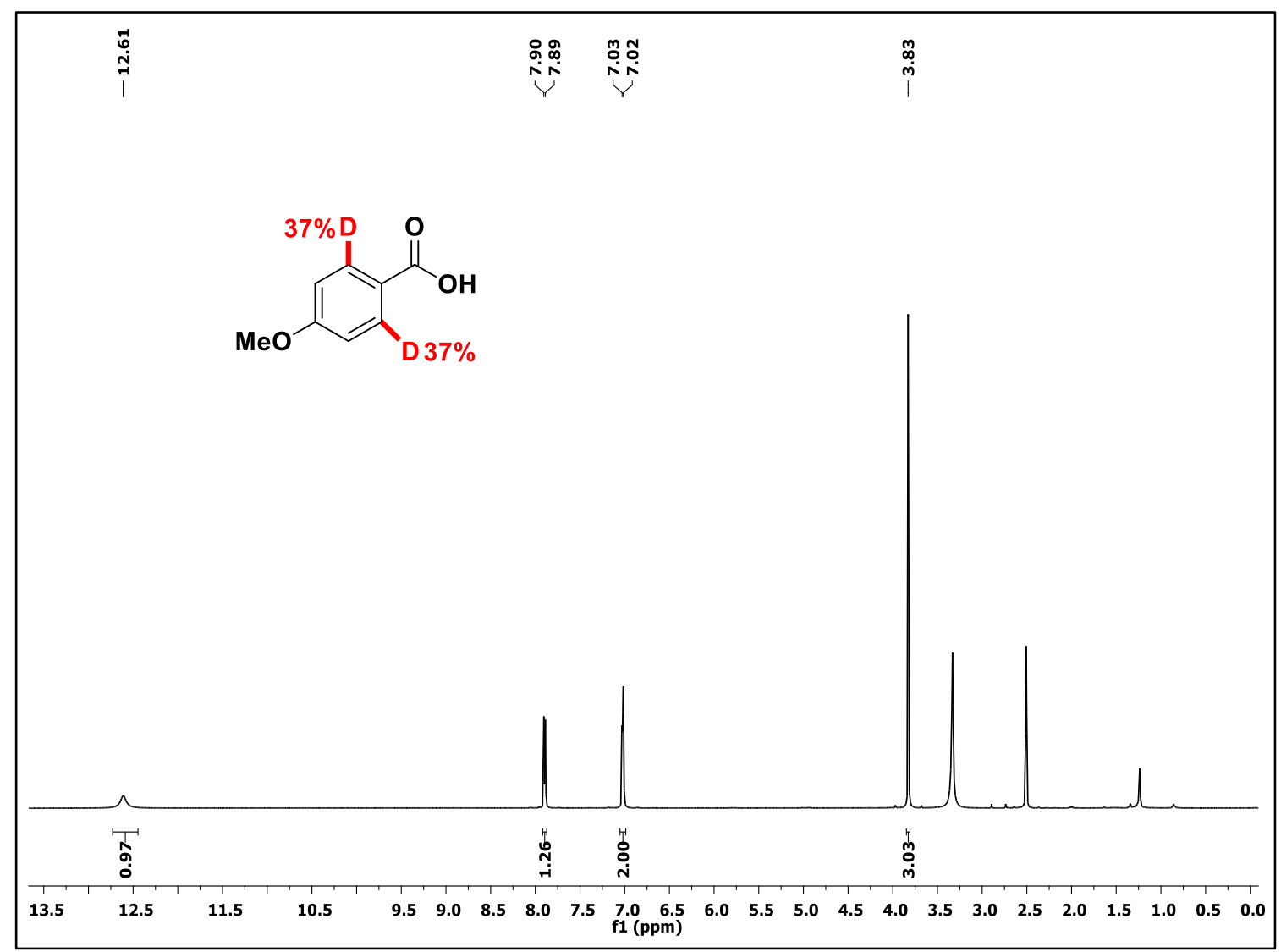

${ }^{1} \mathrm{H}$ Spectra of Compound D-3ja $\left(\mathrm{CDCl}_{3}\right.$ was used).

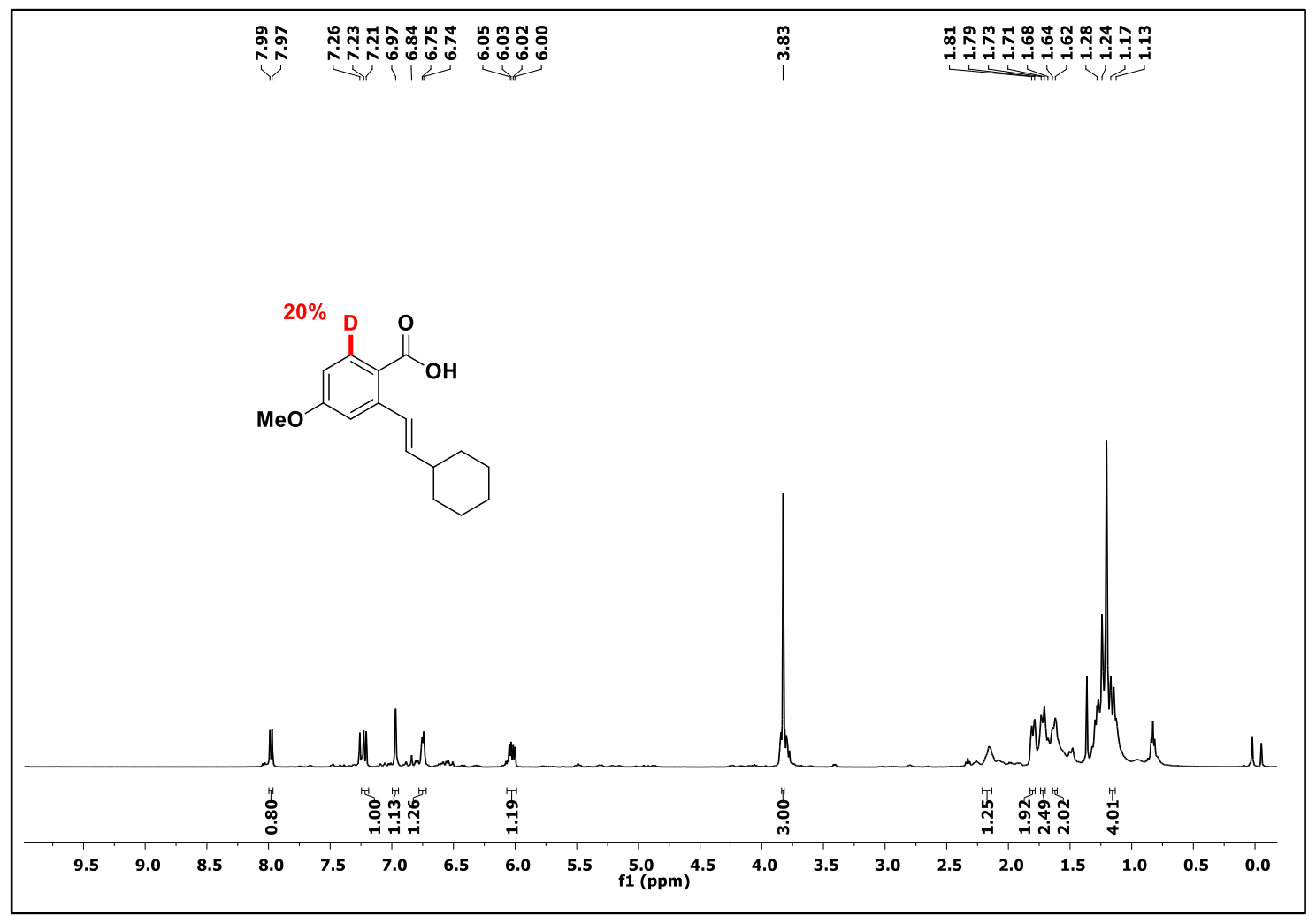


Table S1. Optimization of Olefination Reaction ${ }^{a}$

\begin{tabular}{|c|c|c|c|c|c|c|}
\hline entry & solvent & catalyst & base & oxidant & $\begin{array}{l}\text { tempera } \\
\text { ture }\end{array}$ & $\begin{array}{l}\text { yield } \\
(\%)^{b}\end{array}$ \\
\hline 1 & $\mathrm{DMF}$ & {$\left[(p \text {-cymene }) \mathrm{Ru}(\mathrm{Cl})_{2}\right]_{2}$} & $\mathrm{~K}_{2} \mathrm{CO}_{3}$ & $\mathrm{AgOAc}$ & $100{ }^{\circ} \mathrm{C}$ & NR \\
\hline 2 & DMF & {$\left[\mathrm{Rh}\left(\mathrm{Cp}^{*}\right)\left(\mathrm{Cl}_{2}\right)\right]_{2}$} & $\mathrm{~K}_{2} \mathrm{CO}_{3}$ & $\mathrm{AgOAc}$ & $100{ }^{\circ} \mathrm{C}$ & 48 \\
\hline 3 & $\mathrm{DMF}$ & {$\left[\mathrm{Rh}\left(\mathrm{Cp}^{*}\right)\left(\mathrm{Cl}_{2}\right)\right]_{2}$} & $\mathrm{~K}_{2} \mathrm{CO}_{3}$ & $\mathrm{Ag}_{2} \mathrm{CO}_{3}$ & $100{ }^{\circ} \mathrm{C}$ & 57 \\
\hline 4 & $\mathrm{DMF}$ & {$\left[\mathrm{Rh}\left(\mathrm{Cp}^{*}\right)\left(\mathrm{Cl}_{2}\right)\right]_{2}$} & $\mathrm{~K}_{2} \mathrm{CO}_{3}$ & $\mathrm{Ag}_{2} \mathrm{O}$ & $100{ }^{\circ} \mathrm{C}$ & 65 \\
\hline 5 & $\mathrm{DMF}$ & {$\left[\mathrm{Rh}\left(\mathrm{Cp}^{*}\right)\left(\mathrm{Cl}_{2}\right)\right]_{2}$} & $\mathrm{~K}_{2} \mathrm{CO}_{3}$ & $\begin{array}{c}\mathrm{Cu}(\mathrm{OAc})_{2} . \\
\mathrm{H}_{2} \mathrm{O}\end{array}$ & $100{ }^{\circ} \mathrm{C}$ & 20 \\
\hline 6 & $\mathrm{DMF}$ & {$\left[\mathrm{Rh}\left(\mathrm{Cp}^{*}\right)\left(\mathrm{Cl}_{2}\right)\right]_{2}$} & $\mathrm{~K}_{2} \mathrm{CO}_{3}$ & $\mathrm{Cu}(\mathrm{OAc})_{2}$ & $100{ }^{\circ} \mathrm{C}$ & trace \\
\hline 7 & DMF & {$\left[\mathrm{Rh}\left(\mathrm{Cp}^{*}\right)\left(\mathrm{Cl}_{2}\right)\right]_{2}$} & $\mathrm{~K}_{2} \mathrm{CO}_{3}$ & $\mathrm{CuO}$ & $100{ }^{\circ} \mathrm{C}$ & 20 \\
\hline 8 & $\mathrm{DMF}$ & {$\left[\mathrm{Rh}\left(\mathrm{Cp}^{*}\right)\left(\mathrm{Cl}_{2}\right)\right]_{2}$} & $\mathrm{~K}_{2} \mathrm{CO}_{3}$ & $\mathrm{~K}_{2} \mathrm{~S}_{2} \mathrm{O}_{8}$ & $100{ }^{\circ} \mathrm{C}$ & NR \\
\hline 9 & $\mathrm{DMF}$ & {$\left[\mathrm{Rh}\left(\mathrm{Cp}^{*}\right)\left(\mathrm{Cl}_{2}\right)\right]_{2}$} & $\mathrm{~K}_{2} \mathrm{CO}_{3}$ & $\left(\mathrm{NH}_{4}\right)_{2} \mathrm{~S}_{2} \mathrm{O}$ & $100{ }^{\circ} \mathrm{C}$ & NR \\
\hline 10 & $\mathrm{DMF}$ & {$\left[\mathrm{Rh}\left(\mathrm{Cp}^{*}\right)\left(\mathrm{Cl}_{2}\right)\right]_{2}$} & $\mathrm{Li}_{2} \mathrm{CO}_{3}$ & $\begin{array}{c}8 \\
\mathrm{Ag}_{2} \mathrm{O}\end{array}$ & $100{ }^{\circ} \mathrm{C}$ & 40 \\
\hline 11 & $\mathrm{DMF}$ & {$\left[\mathrm{Rh}\left(\mathrm{Cp}^{*}\right)\left(\mathrm{Cl}_{2}\right)\right]_{2}$} & $\mathrm{Na}_{2} \mathrm{CO}_{3}$ & $\mathrm{Ag}_{2} \mathrm{O}$ & $100{ }^{\circ} \mathrm{C}$ & 40 \\
\hline 12 & DMF & {$\left[\mathrm{Rh}\left(\mathrm{Cp}^{*}\right)\left(\mathrm{Cl}_{2}\right)\right]_{2}$} & $\mathrm{Cs}_{2} \mathrm{CO}_{3}$ & $\mathrm{Ag}_{2} \mathrm{O}$ & $100{ }^{\circ} \mathrm{C}$ & trace \\
\hline 13 & $\mathrm{DMF}$ & {$\left[\mathrm{Rh}\left(\mathrm{Cp}^{*}\right)\left(\mathrm{Cl}_{2}\right)\right]_{2}$} & $\mathrm{Na}_{2} \mathrm{HPO}_{4}$ & $\mathrm{Ag}_{2} \mathrm{O}$ & $100{ }^{\circ} \mathrm{C}$ & 70 \\
\hline 14 & $\mathrm{DMF}$ & {$\left[\mathrm{Rh}\left(\mathrm{Cp}^{*}\right)\left(\mathrm{Cl}_{2}\right)\right]_{2}$} & $\mathrm{KH}_{2} \mathrm{PO}_{4}$ & $\mathrm{Ag}_{2} \mathrm{O}$ & $100{ }^{\circ} \mathrm{C}$ & 52 \\
\hline 15 & $\mathrm{DMF}$ & {$\left[\mathrm{Rh}\left(\mathrm{Cp}^{*}\right)\left(\mathrm{Cl}_{2}\right)\right]_{2}$} & $\mathrm{~K}_{3} \mathrm{PO}_{4}$ & $\mathrm{Ag}_{2} \mathrm{O}$ & $100{ }^{\circ} \mathrm{C}$ & NR \\
\hline 16 & DMF & {$\left[\mathrm{Rh}\left(\mathrm{Cp}^{*}\right)\left(\mathrm{Cl}_{2}\right)\right]_{2}$} & $\mathrm{NaHCO}_{3}$ & $\mathrm{Ag}_{2} \mathrm{O}$ & $100{ }^{\circ} \mathrm{C}$ & 45 \\
\hline 17 & $\mathrm{DMF}$ & {$\left[\mathrm{Rh}\left(\mathrm{Cp}^{*}\right)\left(\mathrm{Cl}_{2}\right)\right]_{2}$} & $\mathrm{KHCO}_{3}$ & $\mathrm{Ag}_{2} \mathrm{O}$ & $100{ }^{\circ} \mathrm{C}$ & trace \\
\hline 18 & $\mathrm{DMF}$ & {$\left[\mathrm{Rh}\left(\mathrm{Cp}^{*}\right)\left(\mathrm{Cl}_{2}\right)\right]_{2}$} & KOAc & $\mathrm{Ag}_{2} \mathrm{O}$ & $100{ }^{\circ} \mathrm{C}$ & trace \\
\hline 19 & $\mathrm{DMF}$ & {$\left[\mathrm{Rh}\left(\mathrm{Cp}^{*}\right)\left(\mathrm{Cl}_{2}\right)\right]_{2}$} & $\mathrm{CsOAc}$ & $\mathrm{Ag}_{2} \mathrm{O}$ & $100{ }^{\circ} \mathrm{C}$ & trace \\
\hline 20 & $\mathrm{DMF}$ & {$\left[\mathrm{Rh}\left(\mathrm{Cp}^{*}\right)\left(\mathrm{Cl}_{2}\right)\right]_{2}$} & $\mathrm{NaOAc}$ & $\mathrm{Ag}_{2} \mathrm{O}$ & $100{ }^{\circ} \mathrm{C}$ & trace \\
\hline 21 & $\mathrm{DMF}$ & {$\left[\mathrm{Rh}\left(\mathrm{Cp}^{*}\right)\left(\mathrm{Cl}_{2}\right)\right]_{2}$} & $\mathrm{~K}_{2} \mathrm{HPO}_{4}$ & $\mathrm{Ag}_{2} \mathrm{O}$ & $100{ }^{\circ} \mathrm{C}$ & trace \\
\hline 22 & $\mathrm{DMF}$ & {$\left[\mathrm{Rh}\left(\mathrm{Cp}^{*}\right)\left(\mathrm{Cl}_{2}\right)\right]_{2}$} & $\mathrm{LiOAc}$ & $\mathrm{Ag}_{2} \mathrm{O}$ & $100{ }^{\circ} \mathrm{C}$ & trace \\
\hline 23 & $\mathrm{DMF}$ & {$\left[\mathrm{Rh}\left(\mathrm{Cp}^{*}\right)\left(\mathrm{Cl}_{2}\right)\right]_{2}$} & $(\mathrm{Cy})_{2} \mathrm{NH}$ & $\mathrm{Ag}_{2} \mathrm{O}$ & $100{ }^{\circ} \mathrm{C}$ & 20 \\
\hline 24 & $\mathrm{DMF}$ & {$\left[\mathrm{Rh}\left(\mathrm{Cp}^{*}\right)\left(\mathrm{Cl}_{2}\right)\right]_{2}$} & $\mathrm{Et}_{3} \mathrm{NH}$ & $\mathrm{Ag}_{2} \mathrm{O}$ & $100{ }^{\circ} \mathrm{C}$ & trace \\
\hline 25 & DMSO & {$\left[\mathrm{Rh}\left(\mathrm{Cp}^{*}\right)\left(\mathrm{Cl}_{2}\right)\right]_{2}$} & $\mathrm{Na}_{2} \mathrm{HPO}_{4}$ & $\mathrm{Ag}_{2} \mathrm{O}$ & $100{ }^{\circ} \mathrm{C}$ & NR \\
\hline 26 & toluene & {$\left[\mathrm{Rh}\left(\mathrm{Cp}^{*}\right)\left(\mathrm{Cl}_{2}\right)\right]_{2}$} & $\mathrm{Na}_{2} \mathrm{HPO}_{4}$ & $\mathrm{Ag}_{2} \mathrm{O}$ & $100{ }^{\circ} \mathrm{C}$ & trace \\
\hline 27 & $\begin{array}{l}\text { Cycloh } \\
\text { exane }\end{array}$ & {$\left[\mathrm{Rh}\left(\mathrm{Cp}^{*}\right)\left(\mathrm{Cl}_{2}\right)\right]_{2}$} & $\mathrm{Na}_{2} \mathrm{HPO}_{4}$ & $\mathrm{Ag}_{2} \mathrm{O}$ & $100{ }^{\circ} \mathrm{C}$ & NR \\
\hline 28 & $\mathrm{CH}_{3} \mathrm{CN}$ & {$\left[\mathrm{Rh}\left(\mathrm{Cp}^{*}\right)\left(\mathrm{Cl}_{2}\right)\right]_{2}$} & $\mathrm{Na}_{2} \mathrm{HPO}_{4}$ & $\mathrm{Ag}_{2} \mathrm{O}$ & $100{ }^{\circ} \mathrm{C}$ & 20 \\
\hline 29 & THF & {$\left[\mathrm{Rh}\left(\mathrm{Cp}^{*}\right)\left(\mathrm{Cl}_{2}\right)\right]_{2}$} & $\mathrm{Na}_{2} \mathrm{HPO}_{4}$ & $\mathrm{Ag}_{2} \mathrm{O}$ & $100{ }^{\circ} \mathrm{C}$ & 25 \\
\hline 30 & $\begin{array}{c}1,4- \\
\text { dioxane }\end{array}$ & {$\left[\mathrm{Rh}\left(\mathrm{Cp}^{*}\right)\left(\mathrm{Cl}_{2}\right)\right]_{2}$} & $\mathrm{Na}_{2} \mathrm{HPO}_{4}$ & $\mathrm{Ag}_{2} \mathrm{O}$ & $100{ }^{\circ} \mathrm{C}$ & trace \\
\hline 31 & DME & {$\left[\mathrm{Rh}\left(\mathrm{Cp}^{*}\right)\left(\mathrm{Cl}_{2}\right)\right]_{2}$} & $\mathrm{Na}_{2} \mathrm{HPO}_{4}$ & $\mathrm{Ag}_{2} \mathrm{O}$ & $100{ }^{\circ} \mathrm{C}$ & \\
\hline
\end{tabular}




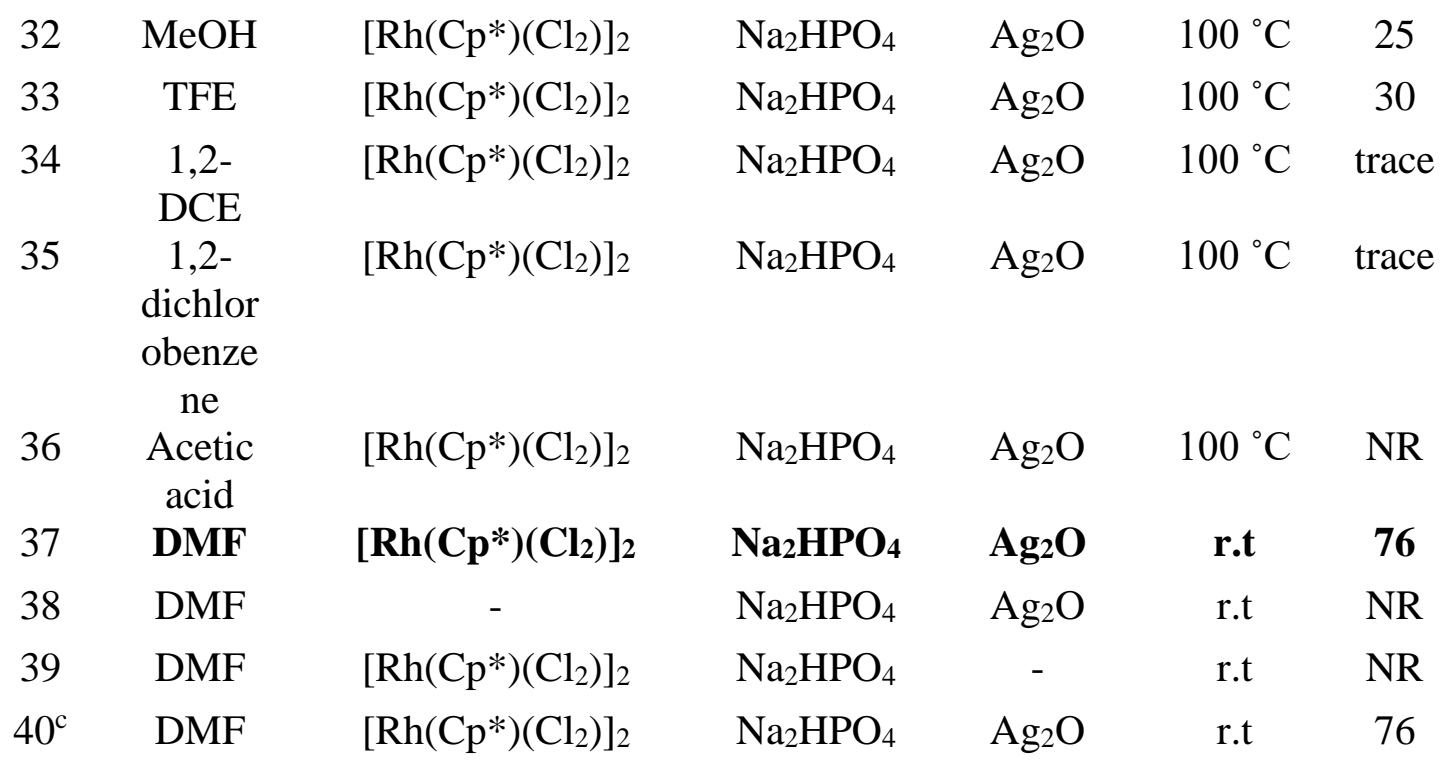

${ }^{a}$ All reactions were carried out using substituted Aromatic Acids 1 (50 mg), alkenes (2) (4.0 equiv), catalyst (5 mol \%), base (1 equiv) and oxidant $(50 \mathrm{~mol} \%)$ in solvent $(3 \mathrm{~mL})$ at $100{ }^{\circ} \mathrm{C}$ or $\mathrm{rt}$ for $24 \mathrm{~h} .{ }^{b}$ Isolated yield. ${ }^{c}$ Aromatic Acids 1 (50 mg), alkenes (2) (4.0 equiv), $\left[\mathrm{Rh}\left(\mathrm{Cp}^{*}\right)\left(\mathrm{Cl}_{2}\right)\right]_{2}(5 \mathrm{~mol} \%), \mathrm{Na}_{2} \mathrm{HPO}_{4}(2$ equiv $)$ and $\mathrm{Ag}_{2} \mathrm{O}(50 \mathrm{~mol} \%)$ in solvent $(3 \mathrm{~mL})$ at $\mathrm{rt}$ for $24 \mathrm{~h}$ 


\section{Crystallographic Data of Compound 3ge}

Suitable single crystals for X-ray diffraction studies were obtained from the compound synthesized in this study. Single crystals were grown in an NMR tube at room temperature using $\mathrm{CDCl}_{3}$ over a period of 3-4 weeks by slow evaporation of solvent.

X-ray data was collected with a Bruker AXS (Kappa Apex 2) CCD diffractometer equipped with graphite monochromatic $\mathrm{Mo}(\mathrm{K} \alpha)(\lambda=0.7107 \mathrm{~A})$ radiation source. The data were collected with $100 \%$ completeness for $\Theta$ up to $25^{\circ}$. $\omega$ and $\phi$ scans were employed to collect the data. The frame width for $\omega$ for was fixed to $0.5^{\circ}$ for data collection. The crystal was solved by direct methods using Bruker SHELXS (Sheldrick, 1997). The Structure was refined using the Bruker SHELXTL (Version 6.12) software package. These data were deposited with Cambridge Crystallographic Data Centre with the following numbers: CCDC 1990502.
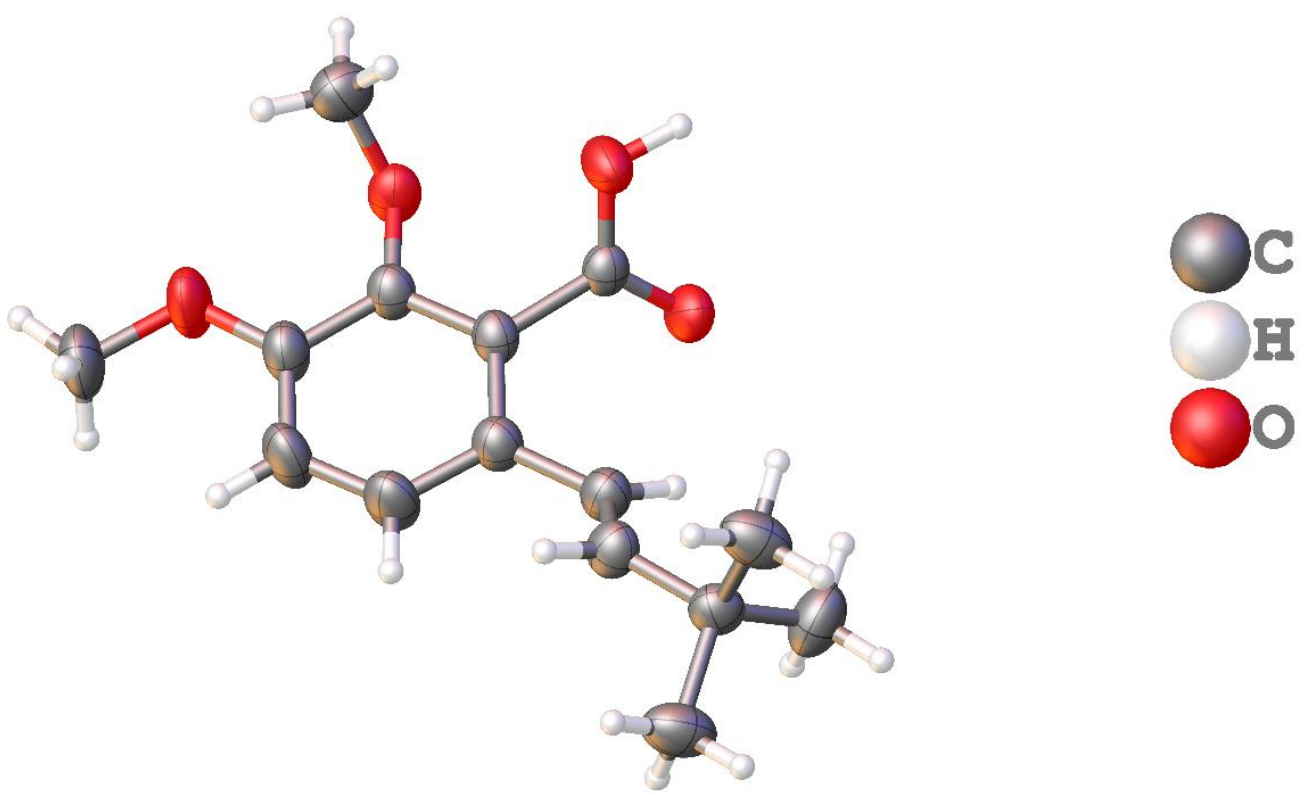

Figure S1: ORTEP representation of compound 3ge displaying thermal elliposoid at 50\% probability. 
X-Ray Analysis of Compounds 3ge

\begin{tabular}{|c|c|}
\hline & 3ge \\
\hline empirical formula & $\mathrm{C}_{15} \mathrm{H}_{20} \mathrm{O}_{4}$ \\
\hline formula wt & 264.31 \\
\hline temp $(K)$ & $296(2)$ \\
\hline Cryst. syst. & triclinic \\
\hline space group & $\mathrm{P}-1$ \\
\hline$a(\AA)$ & $7.8308(2)$ \\
\hline$b(\AA)$ & $9.2666(3)$ \\
\hline$c(\AA)$ & $11.8003(4)$ \\
\hline$\alpha(\operatorname{deg})$ & $70.8979(14)$ \\
\hline$\beta(\operatorname{deg})$ & $76.4643(14)$ \\
\hline$\gamma(\operatorname{deg})$ & $66.1845(14)$ \\
\hline$V\left(\AA^{3}\right)$ & $735.08(4)$ \\
\hline$Z$ & 2 \\
\hline$\rho_{\text {calcd }}\left(\mathrm{Mg} \mathrm{m}^{-3}\right)$ & 1.194 \\
\hline$\mu\left(\mathrm{mm}^{-1}\right)$ & 0.086 \\
\hline$F(000)$ & 284.0 \\
\hline Cryst size (mm) & $0.250 \times 0.220 \times 0.160$ \\
\hline @range (deg) & 3.678 to 49.984 \\
\hline \multirow{2}{*}{$\begin{array}{l}\text { no. of collected/unique } \\
\text { rflns }\end{array}$} & $10824 / 2578$ \\
\hline & {$[\operatorname{Rint}=0.0175]$} \\
\hline $\begin{array}{l}\text { no.of. data /restraints/ } \\
\text { params }\end{array}$ & $2578 / 0 / 182$ \\
\hline$R 1, w R 2(I>2 \sigma(I))$ & $0.0366,0.1004$ \\
\hline$R 1, w R 2$ (all data) & $0.0435,0.1076$ \\
\hline GOF & 1.063 \\
\hline$\Delta \rho_{\max } / \Delta \rho_{\min }\left(\mathrm{e} \AA^{-3}\right)$ & $0.17 /-0.13$ \\
\hline
\end{tabular}




\section{Reference:}

1) White, C.; Yates, A.; Maitlis, P. M. Inorg. Synth. 1992, 29, 228.

2) Sun, R.; Yang, X.; Li, Q.; Xu, K.; Tang, J.; Zheng, X.; Yuan, M.; Fu, H.; Li, R.; Chen, H. Org. Lett. 2019, 21, 9425-9429. 


\section{Spectral Data of Vinylation/Allylation/Branched Alkenes Compounds}

(E)-2-(2-Cyclohexylvinyl)-6-methoxybenzoic acid (3aa).<smiles>COc1cccc(/C=C/C2CCCCC2)c1C(=O)O</smiles>

White solid; eluent (hexane,3\% ethyl acetate, and $1 \% \mathrm{HCOOH}$ ). The reaction scale is $50 \mathrm{mg}$, $64 \mathrm{mg}$ of product was isolated and yield is $76 \%$.

${ }^{1} \mathrm{H}$ NMR $\left(500 \mathrm{MHz}, \mathrm{CDCl}_{3}\right) \delta 7.32(\mathrm{t}, \mathrm{J}=8.1 \mathrm{~Hz}, 1 \mathrm{H}), 7.16(\mathrm{~d}, \mathrm{~J}=7.9 \mathrm{~Hz}, 1 \mathrm{H}), 6.82(\mathrm{~d}, \mathrm{~J}=$ $8.3 \mathrm{~Hz}, 1 \mathrm{H}), 6.60(\mathrm{~d}, \mathrm{~J}=15.7 \mathrm{~Hz}, 1 \mathrm{H}), 6.17$ (dd, J = 15.8, $7.1 \mathrm{~Hz}, 1 \mathrm{H}), 3.89$ (s, 3H), 2.16 (dtt, $\mathrm{J}=13.9,7.0,3.3 \mathrm{~Hz}, 1 \mathrm{H}), 1.85-1.78(\mathrm{~m}, 2 \mathrm{H}), 1.79-1.72(\mathrm{~m}, 2 \mathrm{H}), 1.70-1.63(\mathrm{~m}, 1 \mathrm{H}), 1.37$ -1.27 (m, 2H), $1.24-1.15$ (m, 3H).

${ }^{13} \mathrm{C} \mathrm{NMR}\left(126 \mathrm{MHz}, \mathrm{CDCl}_{3}\right) \delta 171.7,156.7,140.5,138.2,130.9,124.3,120.5,118.6,109.3$, $56.2,41.3,32.7,26.1,25.9$.

HRMS (ESI-TOF) m/z: [M + Na $]^{+}$Calcd for $\mathrm{C}_{16} \mathrm{H}_{20} \mathrm{O}_{3} \mathrm{Na} 283.1310$; Found 283.1307.

(E)-2-(2-Cyclohexylvinyl)-6-methylbenzoic acid (3ba).<smiles>Cc1cccc(/C=C/C2CCCCC2)c1C(=O)O</smiles>

White solid; eluent (hexane,3\% ethyl acetate, and $1 \% \mathrm{HCOOH}$ ). The reaction scale is $50 \mathrm{mg}$, $67 \mathrm{mg}$ of product was isolated and yield is $75 \%$.

${ }^{1} \mathrm{H}$ NMR (500 MHz, $\left.\mathrm{CDCl}_{3}\right) \delta 7.37(\mathrm{~d}, \mathrm{~J}=7.8 \mathrm{~Hz}, 1 \mathrm{H}), 7.26(\mathrm{dd}, \mathrm{J}=8.5,7.0 \mathrm{~Hz}, 1 \mathrm{H}), 7.08$ (d, $\mathrm{J}=7.5 \mathrm{~Hz}, 1 \mathrm{H}), 6.55(\mathrm{~d}, \mathrm{~J}=15.7 \mathrm{~Hz}, 1 \mathrm{H}), 6.14(\mathrm{dd}, \mathrm{J}=15.7,7.0 \mathrm{~Hz}, 1 \mathrm{H}), 2.43(\mathrm{~s}, 3 \mathrm{H}), 2.15$ $(\mathrm{tdd}, \mathrm{J}=10.5,7.0,3.0 \mathrm{~Hz}, 1 \mathrm{H}), 1.84-1.78(\mathrm{~m}, 2 \mathrm{H}), 1.78-1.71(\mathrm{~m}, 2 \mathrm{H}), 1.69-1.64(\mathrm{~m}, 1 \mathrm{H})$, $1.35-1.26(\mathrm{~m}, 2 \mathrm{H}), 1.24-1.15(\mathrm{~m}, 3 \mathrm{H})$.

${ }^{13} \mathrm{C} \mathrm{NMR}\left(126 \mathrm{MHz}, \mathrm{CDCl}_{3}\right) \delta 174.6,140.1,136.0,135.2,131.1,129.8,128.6,124.44,123.3$, 41.3, 32.7, 26.1, 25.9, 19.9 .

HRMS (ESI-TOF) m/z: [M + Na $]^{+}$Calcd for $\mathrm{C}_{16} \mathrm{H}_{20} \mathrm{O}_{2} \mathrm{Na}$ 267.1361; Found 267.1355. 
(E)-2-(2-Cyclohexylvinyl)-6-fluorobenzoic acid (3ca).<smiles>O=C(O)c1c(F)cccc1/C=C/C1CCCCC1</smiles>

White solid; eluent (hexane, $2 \%$ ethyl acetate, and $1 \% \mathrm{HCOOH}$ ). The reaction scale is $50 \mathrm{mg}$, $62 \mathrm{mg}$ of product was isolated and yield is $71 \%$.

${ }^{1} \mathrm{H}$ NMR $\left(500 \mathrm{MHz}, \mathrm{CDCl}_{3}\right) \delta 7.36(\mathrm{qd}, \mathrm{J}=7.8,6.2 \mathrm{~Hz}, 2 \mathrm{H}), 7.03-6.96(\mathrm{~m}, 1 \mathrm{H}), 6.67(\mathrm{~d}, \mathrm{~J}=$ $15.8 \mathrm{~Hz}, 1 \mathrm{H}), 6.19(\mathrm{dd}, \mathrm{J}=15.8,7.1 \mathrm{~Hz}, 1 \mathrm{H}), 2.18(\mathrm{dtd}, \mathrm{J}=14.2,7.2,3.6 \mathrm{~Hz}, 1 \mathrm{H}), 1.85-1.74$ $(\mathrm{m}, 4 \mathrm{H}), 1.68(\mathrm{dd}, \mathrm{J}=9.3,3.4 \mathrm{~Hz}, 1 \mathrm{H}), 1.38-1.27(\mathrm{~m}, 2 \mathrm{H}), 1.24-1.15(\mathrm{~m}, 3 \mathrm{H})$.

${ }^{13} \mathrm{C} \mathrm{NMR}\left(126 \mathrm{MHz}, \mathrm{CDCl}_{3}\right) \delta 170.7,161.4,159.4,141.6,139.6,131.9,131.9,123.6,123.6$, $121.8,121.7,118.6,118.5,114.0,113.8,41.3,32.6,26.0,25.9$.

HRMS (ESI-TOF) m/z: [M + Na $]^{+}$Calcd for $\mathrm{C}_{15} \mathrm{H}_{17} \mathrm{FO}_{2} \mathrm{Na} 271.1110$; Found 271.1102.

(E)-2-Chloro-6-(2-cyclohexylvinyl)benzoic acid (3da).<smiles>O=C(O)c1c(Cl)cccc1/C=C/C1CCCCC1</smiles>

White solid; eluent (hexane,5\% ethyl acetate, and $1 \% \mathrm{HCOOH}$ ). The reaction scale is $50 \mathrm{mg}$, $52 \mathrm{mg}$ of product was isolated and yield is $62 \%$.

${ }^{1} \mathrm{H}$ NMR (500 MHz, DMSO) $\delta 7.61-7.56(\mathrm{~m}, 1 \mathrm{H}), 7.39-7.34(\mathrm{~m}, 2 \mathrm{H}), 6.36(\mathrm{dd}, \mathrm{J}=15.9$, $6.6 \mathrm{~Hz}, 1 \mathrm{H}), 6.28(\mathrm{~d}, \mathrm{~J}=15.9 \mathrm{~Hz}, 1 \mathrm{H}), 2.19-2.10(\mathrm{~m}, 1 \mathrm{H}), 1.77-1.67(\mathrm{~m}, 4 \mathrm{H}), 1.67-1.59$ $(\mathrm{m}, 1 \mathrm{H}), 1.28(\mathrm{ddd}, \mathrm{J}=12.6,7.8,3.1 \mathrm{~Hz}, 2 \mathrm{H}), 1.20-1.10(\mathrm{~m}, 3 \mathrm{H})$.

${ }^{13} \mathrm{C}$ NMR (126 MHz, DMSO) $\delta 168.3,141.1,136.1,134.0,130.6,129.4,127.7,124.1,123.3$, 41.0, 32.6, 26.0, 25.7.

Compound (E)-2-Chloro-6-(2-cyclohexylvinyl)benzoic acid having poor ionization under ESIMS condition for HRMS. Hence, the acid group was converted into Methyl (E)-2-chloro-6-(2cyclohexylvinyl)benzoate and HRMS was recorded. The preparation of ester from acid was given in page number: $\mathrm{S} 3$.

HRMS (ESI-TOF) m/z: [M + Na] ${ }^{+}$Calcd for $\mathrm{C}_{16} \mathrm{H}_{19} \mathrm{ClO}_{2} \mathrm{Na}$ 301.0971; Found 301.0963. 
(E)-2-Bromo-6-(2-cyclohexylvinyl)benzoic acid (3ea).<smiles>O=C(O)c1c(Br)cccc1/C=C/C1CCCCC1</smiles>

White solid; eluent (hexane, $4 \%$ ethyl acetate, and $1 \% \mathrm{HCOOH}$ ). The reaction scale is $50 \mathrm{mg}$, $55 \mathrm{mg}$ of product was isolated and yield is $73 \%$.

${ }^{1} \mathrm{H}$ NMR (500 MHz, DMSO) $\delta 7.62(\mathrm{~d}, \mathrm{~J}=7.9 \mathrm{~Hz}, 1 \mathrm{H}), 7.50(\mathrm{~d}, \mathrm{~J}=7.9 \mathrm{~Hz}, 1 \mathrm{H}), 7.29(\mathrm{t}, \mathrm{J}=$ $8.0 \mathrm{~Hz}, 1 \mathrm{H}), 6.35(\mathrm{dd}, \mathrm{J}=15.9,6.5 \mathrm{~Hz}, 1 \mathrm{H}), 6.28(\mathrm{~d}, \mathrm{~J}=16.0 \mathrm{~Hz}, 1 \mathrm{H}), 2.19-2.09(\mathrm{~m}, 1 \mathrm{H})$, $1.77-1.67(\mathrm{~m}, 4 \mathrm{H}), 1.63(\mathrm{~d}, \mathrm{~J}=12.6 \mathrm{~Hz}, 1 \mathrm{H}), 1.35-1.22(\mathrm{~m}, 2 \mathrm{H}), 1.20-1.09(\mathrm{~m}, 3 \mathrm{H})$.

${ }^{13} \mathrm{C}$ NMR (126 MHz, DMSO) $\delta$ 169.0, 141.0, 136.2, 130.9, 130.8, 124.5, 123.4, 118.96, 118.4, 41.0, 32.6, 26.0, 25.7.

Compound (E)-2-Bromo-6-(2-cyclohexylvinyl)benzoic acid having poor ionization under ESIMS condition for HRMS. Hence, the acid group was converted into Methyl (E)-2-bromo-6-(2cyclohexylvinyl)benzoate and HRMS was measured. The preparation of ester from acid was given in page number: $\mathrm{S} 3$.

HRMS (ESI-TOF) m/z: [M + Na $]^{+}$Calcd for $\mathrm{C}_{16} \mathrm{H}_{19} \mathrm{BrO}_{2} \mathrm{Na} 345.0466$; Found 345.0458.

(E)-3-(2-Cyclohexylvinyl)-[1,1'-biphenyl]-2-carboxylic acid (3fa).<smiles>O=C(O)c1c(/C=C/C2CCCCC2)cccc1-c1ccccc1</smiles>

White solid; eluent (hexane,5\% ethyl acetate, and 1\% $\mathrm{HCOOH}$ ). The reaction scale is $50 \mathrm{mg}$, $40 \mathrm{mg}$ of product was isolated and yield is $52 \%$.

${ }^{1} \mathrm{H}$ NMR $\left(500 \mathrm{MHz}, \mathrm{CDCl}_{3}\right) \delta 7.54(\mathrm{~d}, \mathrm{~J}=7.7 \mathrm{~Hz}, 1 \mathrm{H}), 7.43-7.34(\mathrm{~m}, 6 \mathrm{H}), 7.25-7.20(\mathrm{~m}$, $1 \mathrm{H}), 6.56(\mathrm{~d}, \mathrm{~J}=15.7 \mathrm{~Hz}, 1 \mathrm{H}), 6.20(\mathrm{dd}, \mathrm{J}=15.7,7.1 \mathrm{~Hz}, 1 \mathrm{H}), 2.19-2.12(\mathrm{~m}, 1 \mathrm{H}), 1.85-1.74$ $(\mathrm{m}, 4 \mathrm{H}), 1.71-1.65(\mathrm{~m}, 1 \mathrm{H}), 1.32(\mathrm{ddd}, \mathrm{J}=12.7,7.8,2.9 \mathrm{~Hz}, 2 \mathrm{H}), 1.20$ (ddd, J = 11.8, 7.6, $3.4 \mathrm{~Hz}, 3 \mathrm{H})$.

${ }^{13} \mathrm{C}$ NMR $\left(126 \mathrm{MHz}, \mathrm{CDCl}_{3}\right) \delta 173.7,140.6,140.5,140.2,136.1,129.7,128.4,128.3,127.5$, 124.6, 124.0, 41.3, 32.7, 26.1, 25.9.

HRMS (ESI-TOF) m/z: [M + Na $]^{+}$Calcd for $\mathrm{C}_{21} \mathrm{H}_{22} \mathrm{O}_{2} \mathrm{Na} 329.1517$; Found 329.1524. 
(E)-6-(2-Cyclohexylvinyl)-2,3-dimethoxybenzoic acid (3ga).<smiles>COc1ccc(/C=C/C2CCCCC2)c(C(=O)O)c1OC</smiles>

White solid; eluent (hexane, $3 \%$ ethyl acetate, and $1 \% \mathrm{HCOOH}$ ). The reaction scale is $50 \mathrm{mg}$, $65 \mathrm{mg}$ of product was isolated and yield is $82 \%$.

${ }^{1} \mathrm{H}$ NMR $\left(500 \mathrm{MHz}, \mathrm{CDCl}_{3}\right) \delta 7.24(\mathrm{~d}, \mathrm{~J}=8.7 \mathrm{~Hz}, 1 \mathrm{H}), 6.95(\mathrm{t}, \mathrm{J}=7.9 \mathrm{~Hz}, 1 \mathrm{H}), 6.51(\mathrm{~d}, \mathrm{~J}=$ $15.8 \mathrm{~Hz}, 1 \mathrm{H}), 6.04(\mathrm{dd}, \mathrm{J}=15.8,7.1 \mathrm{~Hz}, 1 \mathrm{H}), 3.92(\mathrm{~d}, \mathrm{~J}=3.7 \mathrm{~Hz}, 3 \mathrm{H}), 3.89-3.87(\mathrm{~m}, 3 \mathrm{H})$, 2.13 (ddd, J = 10.0, 8.6, $4.7 \mathrm{~Hz}, 1 \mathrm{H}), 1.83-1.72(\mathrm{~m}, 4 \mathrm{H}), 1.66(\mathrm{~d}, \mathrm{~J}=12.6 \mathrm{~Hz}, 1 \mathrm{H}), 1.31$ (ddd, $\mathrm{J}=15.5,12.5,2.9 \mathrm{~Hz}, 2 \mathrm{H}), 1.18(\mathrm{ddd}, \mathrm{J}=21.3,12.0,9.4 \mathrm{~Hz}, 3 \mathrm{H})$.

${ }^{13} \mathrm{C}$ NMR (126 MHz, CDCl3) $\delta 171.2,151.2,146.1,138.6,129.8,123.7,121.9,119.0,114.5$, $61.7,56.1,41.2,32.8,26.1,25.9$.

HRMS (ESI-TOF) m/z: [M + Na $]^{+}$Calcd for $\mathrm{C}_{17} \mathrm{H}_{22} \mathrm{O}_{4} \mathrm{Na} 313.1416$; Found 313.1403.

(E)-2-(2-Cyclohexylvinyl)-4,6-dimethoxybenzoic acid (3ha).<smiles>COc1cc(/C=C/C2CCCCC2)c(C(=O)O)c(OC)c1</smiles>

White solid; eluent (hexane,3\% ethyl acetate, and 1\% $\mathrm{HCOOH}$ ). The reaction scale is $50 \mathrm{mg}$, $54 \mathrm{mg}$ of product was isolated and yield is $68 \%$.

${ }^{1} \mathrm{H}$ NMR $\left(500 \mathrm{MHz}, \mathrm{CDCl}_{3}\right) \delta 6.81(\mathrm{~d}, \mathrm{~J}=15.7 \mathrm{~Hz}, 1 \mathrm{H}), 6.64(\mathrm{~d}, \mathrm{~J}=2.2 \mathrm{~Hz}, 1 \mathrm{H}), 6.39(\mathrm{~d}, \mathrm{~J}=$ $2.2 \mathrm{~Hz}, 1 \mathrm{H}), 6.08(\mathrm{dd}, \mathrm{J}=15.7,6.9 \mathrm{~Hz}, 1 \mathrm{H}), 3.89(\mathrm{~s}, 3 \mathrm{H}), 3.86(\mathrm{~s}, 3 \mathrm{H}), 2.16$ (tdd, J = 12.1, 7.9, $4.3 \mathrm{~Hz}, 1 \mathrm{H}), 1.85-1.79(\mathrm{~m}, 2 \mathrm{H}), 1.78-1.72(\mathrm{~m}, 2 \mathrm{H}), 1.67(\mathrm{ddd}, \mathrm{J}=5.2,4.2,2.4 \mathrm{~Hz}, 1 \mathrm{H})$, 1.30 (ddd, $\mathrm{J}=15.5,9.4,3.1 \mathrm{~Hz}, 2 \mathrm{H}), 1.17$ (qd, J = 6.6, 3.1 Hz, 3H).

${ }^{13} \mathrm{C}$ NMR $\left(126 \mathrm{MHz}, \mathrm{CDCl}_{3}\right) \delta 169.5,162.1,158.9,140.0,125.8,118.9,112.3,103.6,97.4$, $56.4,55.4,41.1,32.7,26.1,25.9$.

HRMS (ESI-TOF) m/z: [M + Na $]^{+}$Calcd for $\mathrm{C}_{17} \mathrm{H}_{22} \mathrm{O}_{4} \mathrm{Na} 313.1416$; Found 313.1420. 
2-(2-Cyclohexylideneethyl)-3,6-dimethoxybenzoic acid (3ia).<smiles>COc1ccc(OC)c(C(=O)O)c1CC=C1CCCCC1</smiles>

White solid; eluent (hexane, $3 \%$ ethyl acetate, and $1 \% \mathrm{HCOOH}$ ). The reaction scale is $50 \mathrm{mg}$, $60 \mathrm{mg}$ of product was isolated and yield is $75 \%$.

${ }^{1} \mathrm{H}$ NMR $\left(500 \mathrm{MHz}, \mathrm{CDCl}_{3}\right) \delta 6.87(\mathrm{~d}, \mathrm{~J}=8.9 \mathrm{~Hz}, 1 \mathrm{H}), 6.76(\mathrm{~d}, \mathrm{~J}=8.9 \mathrm{~Hz}, 1 \mathrm{H}), 5.13(\mathrm{t}, \mathrm{J}=$ $7.0 \mathrm{~Hz}, 1 \mathrm{H}), 3.83(\mathrm{~s}, 3 \mathrm{H}), 3.79(\mathrm{~s}, 3 \mathrm{H}), 3.46(\mathrm{~d}, \mathrm{~J}=7.1 \mathrm{~Hz}, 2 \mathrm{H}), 2.28(\mathrm{~s}, 2 \mathrm{H}), 2.07-2.00(\mathrm{~m}$, $2 \mathrm{H}), 1.57-1.46(\mathrm{~m}, 6 \mathrm{H})$.

${ }^{13} \mathrm{C} \mathrm{NMR}\left(126 \mathrm{MHz}, \mathrm{CDCl}_{3}\right) \delta 171.3,151.9,150.3,139.9,130.0,123.5,118.7,112.8,109.4$, $56.5,56.2,37.1,28.8,28.4,27.7,26.9,25.8$.

HRMS (ESI-TOF) m/z: [M + Na $]^{+}$Calcd for $\mathrm{C}_{17} \mathrm{H}_{22} \mathrm{O}_{4} \mathrm{Na}$ 313.1416; Found 313.1422.

(E)-2-(2-Cyclohexylvinyl)-4-methoxybenzoic acid (3ja).<smiles>COc1ccc(C(=O)O)c(/C=C/C2CCCCC2)c1</smiles>

White solid; eluent (hexane, $3 \%$ ethyl acetate, and $1 \% \mathrm{HCOOH}$ ). The reaction scale is $50 \mathrm{mg}$, $30 \mathrm{mg}$ of product was isolated and yield is $35 \%$.

${ }^{1} \mathrm{H}$ NMR $\left(500 \mathrm{MHz}, \mathrm{CDCl}_{3}\right) \delta 8.03(\mathrm{~d}, \mathrm{~J}=8.7 \mathrm{~Hz}, 1 \mathrm{H}), 7.30(\mathrm{~d}, \mathrm{~J}=15.8 \mathrm{~Hz}, 1 \mathrm{H}), 7.02(\mathrm{~s}, 1 \mathrm{H})$, $6.80(\mathrm{~d}, \mathrm{~J}=8.7 \mathrm{~Hz}, 1 \mathrm{H}), 6.08(\mathrm{dd}, \mathrm{J}=15.8,6.8 \mathrm{~Hz}, 1 \mathrm{H}), 3.88(\mathrm{~s}, 3 \mathrm{H}), 2.20$ (dd, J = 10.9, 7.3 $\mathrm{Hz}, 1 \mathrm{H}), 1.85(\mathrm{~d}, \mathrm{~J}=12.4 \mathrm{~Hz}, 2 \mathrm{H}), 1.78(\mathrm{~d}, \mathrm{~J}=12.8 \mathrm{~Hz}, 2 \mathrm{H}), 1.68(\mathrm{~d}, \mathrm{~J}=12.1 \mathrm{~Hz}, 1 \mathrm{H}), 1.34$ $(\mathrm{dd}, \mathrm{J}=25.0,12.5 \mathrm{~Hz}, 2 \mathrm{H}), 1.26-1.17(\mathrm{~m}, 3 \mathrm{H})$.

${ }^{13} \mathrm{C} \mathrm{NMR}\left(126 \mathrm{MHz}, \mathrm{CDCl}_{3}\right) \delta 172.0,163.0,143.6,139.9,133.8,126.6,119.2,112.4,112.0$, $55.4,41.1,32.8,26.2,26.0$.

HRMS (ESI-TOF) m/z: [M + Na $]^{+}$Calcd for $\mathrm{C}_{16} \mathrm{H}_{20} \mathrm{O}_{3} \mathrm{Na} 283.1310$; Found 283.1307. 
(E)-2-(2-Cyclohexylvinyl)-4-fluorobenzoic acid (3ka).<smiles>O=C(O)c1ccc(F)cc1/C=C/C1CCCCC1</smiles>

White solid; eluent (hexane, $3 \%$ ethyl acetate, and $1 \% \mathrm{HCOOH}$ ). The reaction scale is $50 \mathrm{mg}$, $50 \mathrm{mg}$ of product was isolated and yield is $57 \%$.

${ }^{1} \mathrm{H}$ NMR $\left(500 \mathrm{MHz}, \mathrm{CDCl}_{3}\right) \delta 8.05(\mathrm{dd}, \mathrm{J}=8.6,6.1 \mathrm{~Hz}, 1 \mathrm{H}), 7.28-7.23(\mathrm{~m}, 2 \mathrm{H}), 6.97(\mathrm{td}, \mathrm{J}$ $=8.5,2.5 \mathrm{~Hz}, 1 \mathrm{H}), 6.12(\mathrm{dd}, \mathrm{J}=15.8,6.9 \mathrm{~Hz}, 1 \mathrm{H}), 2.27-2.15(\mathrm{~m}, 1 \mathrm{H}), 1.79(\mathrm{ddd}, \mathrm{J}=16.9$, 12.5, $7.8 \mathrm{~Hz}, 4 \mathrm{H}), 1.69(\mathrm{dd}, \mathrm{J}=9.5,3.3 \mathrm{~Hz}, 1 \mathrm{H}), 1.32$ (dq, J = 18.8, 3.1 Hz, 2H), 1.20 (ddd, J $=12.3,9.3,6.0 \mathrm{~Hz}, 3 \mathrm{H})$.

${ }^{13} \mathrm{C} \mathrm{NMR}\left(126 \mathrm{MHz}, \mathrm{CDCl}_{3}\right) \delta 171.5,166.4,164.4,144.3,144.3,141.3,134.2,134.1,125.5$, $122.8,114.0,113.8,113.8,113.6,41.2,32.7,26.1,25.9$.

HRMS (ESI-TOF) m/z: [M + Na $]^{+}$Calcd for $\mathrm{C}_{15} \mathrm{H}_{17} \mathrm{FO}_{2} \mathrm{Na} 271.1110$; Found 271.1124.

\section{(E)-2-(2-Cyclohexylvinyl)thiophene-3-carboxylic acid (3la).}<smiles>O=C(O)c1ccsc1/C=C/C1CCCCC1</smiles>

White solid; eluent (hexane, 3\% ethyl acetate, and 1\% $\mathrm{HCOOH}$ ). The reaction scale is $50 \mathrm{mg}$, $50 \mathrm{mg}$ of product was isolated and yield is $55 \%$.

${ }^{1} \mathrm{H}$ NMR $\left(500 \mathrm{MHz}, \mathrm{CDCl}_{3}\right) \delta 7.45(\mathrm{~d}, \mathrm{~J}=5.3 \mathrm{~Hz}, 1 \mathrm{H}), 7.34-7.27(\mathrm{~m}, 2 \mathrm{H}), 6.26(\mathrm{dd}, \mathrm{J}=$ 16.1, 7.2 Hz, 1H), $2.20(\mathrm{dtt}, \mathrm{J}=17.3,7.0,3.3 \mathrm{~Hz}, 1 \mathrm{H}), 1.88-1.74(\mathrm{~m}, 4 \mathrm{H}), 1.72-1.66$ (m, 1H), $1.38-1.29(\mathrm{~m}, 2 \mathrm{H}), 1.27-1.16(\mathrm{~m}, 3 \mathrm{H})$.

${ }^{13} \mathrm{C}$ NMR $\left(126 \mathrm{MHz}, \mathrm{CDCl}_{3}\right) \delta 168.1,147.6,142.4,131.7,126.6,124.5,121.1,41.5,32.7$, 26.1, 25.9.

HRMS (ESI-TOF) m/z: [M + Na $]^{+}$Calcd for $\mathrm{C}_{13} \mathrm{H}_{16} \mathrm{SO}_{2} \mathrm{Na} 259.0769$; Found 259.0775. 
(E)-2-(2-Cyclohexylvinyl)-5-methoxybenzoic acid (3ma).<smiles>COc1ccc(/C=C/C2CCCCC2)c(C(=O)O)c1</smiles>

White solid; eluent (hexane, $3 \%$ ethyl acetate, and $1 \% \mathrm{HCOOH}$ ). The reaction scale is $50 \mathrm{mg}$, $48 \mathrm{mg}$ of product was isolated and yield is $57 \%$.

${ }^{1} \mathrm{H}$ NMR $\left(500 \mathrm{MHz}, \mathrm{CDCl}_{3}\right) \delta 7.50(\mathrm{dd}, \mathrm{J}=5.8,2.9 \mathrm{~Hz}, 2 \mathrm{H}), 7.14(\mathrm{~d}, \mathrm{~J}=15.8 \mathrm{~Hz}, 1 \mathrm{H}), 7.05$ $(\mathrm{dd}, \mathrm{J}=8.7,2.8 \mathrm{~Hz}, 1 \mathrm{H}), 6.00(\mathrm{dd}, \mathrm{J}=15.9,6.9 \mathrm{~Hz}, 1 \mathrm{H}), 3.84(\mathrm{~s}, 3 \mathrm{H}), 2.17$ (ddd, $\mathrm{J}=10.4,7.1$, $3.7 \mathrm{~Hz}, 1 \mathrm{H}), 1.83(\mathrm{~d}, \mathrm{~J}=12.6 \mathrm{~Hz}, 2 \mathrm{H}), 1.79-1.74(\mathrm{~m}, 2 \mathrm{H}), 1.68(\mathrm{dd}, \mathrm{J}=9.3,3.5 \mathrm{~Hz}, 1 \mathrm{H}), 1.33$ $(\mathrm{dt}, \mathrm{J}=12.7,3.1 \mathrm{~Hz}, 2 \mathrm{H}), 1.22-1.17(\mathrm{~m}, 3 \mathrm{H})$.

${ }^{13} \mathrm{C} \mathrm{NMR}\left(126 \mathrm{MHz}, \mathrm{CDCl}_{3}\right) \delta 172.1,158.0,138.4,133.5,128.7,127.6,125.6,119.8,114.8$, $55.5,41.2,32.9,26.2,26.0$.

HRMS (ESI-TOF) m/z: [M + Na $]^{+}$Calcd for $\mathrm{C}_{16} \mathrm{H}_{20} \mathrm{O}_{3} \mathrm{Na} 283.1310$; Found 283.1314.

(E)-2-(2-Cyclohexylvinyl)-5-methylbenzoic acid (3na).<smiles>Cc1ccc(/C=C/C2CCCCC2)c(C(=O)O)c1</smiles>

White solid; eluent (hexane, 3\% ethyl acetate, and 1\% $\mathrm{HCOOH}$ ). The reaction scale is $50 \mathrm{mg}$, $66 \mathrm{mg}$ of product was isolated and yield is $75 \%$.

${ }^{1} \mathrm{H}$ NMR $\left(500 \mathrm{MHz}, \mathrm{CDCl}_{3}\right) \delta 7.80(\mathrm{~d}, \mathrm{~J}=0.9 \mathrm{~Hz}, 1 \mathrm{H}), 7.46(\mathrm{~d}, \mathrm{~J}=8.0 \mathrm{~Hz}, 1 \mathrm{H}), 7.29(\mathrm{~d}, \mathrm{~J}=$ $6.7 \mathrm{~Hz}, 1 \mathrm{H}), 7.17(\mathrm{~d}, \mathrm{~J}=15.8 \mathrm{~Hz}, 1 \mathrm{H}), 6.06(\mathrm{dd}, \mathrm{J}=15.8,6.9 \mathrm{~Hz}, 1 \mathrm{H}), 2.37$ (s, 3H), 2.18 (ddd, $\mathrm{J}=10.4,8.6,5.2 \mathrm{~Hz}, 1 \mathrm{H}), 1.84(\mathrm{~d}, \mathrm{~J}=12.6 \mathrm{~Hz}, 2 \mathrm{H}), 1.80-1.74(\mathrm{~m}, 2 \mathrm{H}), 1.71-1.66(\mathrm{~m}, 1 \mathrm{H})$, 1.32 (ddd, $\mathrm{J}=12.5,8.6,3.1 \mathrm{~Hz}, 2 \mathrm{H}), 1.24-1.17(\mathrm{~m}, 3 \mathrm{H})$.

${ }^{13} \mathrm{C} \mathrm{NMR}\left(126 \mathrm{MHz}, \mathrm{CDCl}_{3}\right) \delta 171.8,139.2,138.0,136.3,133.6,131.5,127.3,126.6,125.9$, $41.2,32.9,26.2,26.0,20.9$.

HRMS (ESI-TOF) m/z: [M + Na $]^{+}$Calcd for $\mathrm{C}_{16} \mathrm{H}_{20} \mathrm{O}_{2} \mathrm{Na}$ 267.1361; Found 267.1362. 
(E)-5-Bromo-2-(2-cyclohexylvinyl)benzoic acid (3oa).<smiles>O=C(O)c1cc(Br)ccc1/C=C/C1CCCCC1</smiles>

White solid; eluent (hexane, $3 \%$ ethyl acetate, and $1 \% \mathrm{HCOOH}$ ). The reaction scale is $50 \mathrm{mg}$, $42 \mathrm{mg}$ of product was isolated and yield is $55 \%$.

${ }^{1} \mathrm{H}$ NMR $\left(500 \mathrm{MHz}, \mathrm{CDCl}_{3}\right) \delta 8.13(\mathrm{~d}, \mathrm{~J}=2.1 \mathrm{~Hz}, 1 \mathrm{H}), 7.59(\mathrm{dd}, \mathrm{J}=8.4,2.1 \mathrm{~Hz}, 1 \mathrm{H}), 7.44(\mathrm{~d}$, $\mathrm{J}=8.5 \mathrm{~Hz}, 1 \mathrm{H}), 7.15(\mathrm{~d}, \mathrm{~J}=15.8 \mathrm{~Hz}, 1 \mathrm{H}), 6.11(\mathrm{dd}, \mathrm{J}=15.9,6.9 \mathrm{~Hz}, 1 \mathrm{H}), 2.19(\mathrm{ddd}, \mathrm{J}=13.9$, $8.4,5.1 \mathrm{~Hz}, 1 \mathrm{H}), 1.79(\mathrm{ddd}, \mathrm{J}=16.3,12.7,7.9 \mathrm{~Hz}, 4 \mathrm{H}), 1.72-1.66(\mathrm{~m}, 1 \mathrm{H}), 1.36-1.28(\mathrm{~m}$, $2 \mathrm{H}), 1.24-1.15(\mathrm{~m}, 3 \mathrm{H})$.

${ }^{13} \mathrm{C} \mathrm{NMR}\left(126 \mathrm{MHz}, \mathrm{CDCl}_{3}\right) \delta 171.4,140.9,139.8,135.7,133.9,129.0,128.2,125.2,120.0$, $41.3,32.7,26.1,25.9$.

HRMS (ESI-TOF) m/z: [M + Na $]^{+}$Calcd for $\mathrm{C}_{15} \mathrm{H}_{17} \mathrm{BrO}_{2} \mathrm{Na} 331.0296$; Found 331.0258.

(E)-2-(2-Cyclohexylvinyl)-5-nitrobenzoic acid (3pa).<smiles>O=C(O)c1cc([N+](=O)[O-])ccc1/C=C/C1CCCCC1</smiles>

White solid; eluent (hexane, $2 \%$ ethyl acetate, and $1 \% \mathrm{HCOOH}$ ). The reaction scale is $50 \mathrm{mg}$, $35 \mathrm{mg}$ of product was isolated and yield is $43 \%$.

${ }^{1} \mathrm{H}$ NMR (500 MHz, CDCl $) \delta 8.87(\mathrm{~s}, 1 \mathrm{H}), 8.31(\mathrm{~d}, \mathrm{~J}=7.8 \mathrm{~Hz}, 1 \mathrm{H}), 7.75(\mathrm{~d}, \mathrm{~J}=8.7 \mathrm{~Hz}, 1 \mathrm{H})$, $7.32(\mathrm{~d}, \mathrm{~J}=15.9 \mathrm{~Hz}, 1 \mathrm{H}), 6.33(\mathrm{dd}, \mathrm{J}=15.8,6.8 \mathrm{~Hz}, 1 \mathrm{H}), 2.25(\mathrm{~m}, 1 \mathrm{H}), 1.86(\mathrm{~d}, \mathrm{~J}=12.1 \mathrm{~Hz}$, $1 \mathrm{H}), 1.80(\mathrm{~d}, \mathrm{~J}=12.8 \mathrm{~Hz}, 2 \mathrm{H}), 1.69(\mathrm{~s}, 1 \mathrm{H}), 1.41-1.30(\mathrm{~m}, 2 \mathrm{H}), 1.22(\mathrm{dd}, \mathrm{J}=23.0,11.1 \mathrm{~Hz}$, $4 \mathrm{H})$.

${ }^{13} \mathrm{C} \mathrm{NMR}\left(126 \mathrm{MHz}, \mathrm{CDCl}_{3}\right) \delta 170.1,147.0,145.9,144.9,128.3,127.0,126.8,125.1,124.7$, 41.6, 32.5, 26.0, 25.8 .

HRMS (ESI-TOF) m/z: [M + Na $]^{+}$Calcd for $\mathrm{C}_{15} \mathrm{H}_{17} \mathrm{NO}_{4} \mathrm{Na} 298.1055$; Found 298.1057. 
(E)-2-(2-Cyclohexylvinyl)-4,5-dimethoxybenzoic acid (3qa).<smiles>COc1cc(/C=C/C2CCCCC2)c(C(=O)O)cc1OC</smiles>

White solid; eluent (hexane, 3\% ethyl acetate, and 1\% $\mathrm{HCOOH}$ ). The reaction scale is $50 \mathrm{mg}$, $57 \mathrm{mg}$ of product was isolated and yield is $72 \%$.

${ }^{1} \mathrm{H}$ NMR $\left(500 \mathrm{MHz}, \mathrm{CDCl}_{3}\right) \delta 7.55(\mathrm{~s}, 1 \mathrm{H}), 7.31(\mathrm{~d}, \mathrm{~J}=15.8 \mathrm{~Hz}, 1 \mathrm{H}), 7.00(\mathrm{~s}, 1 \mathrm{H}), 6.02(\mathrm{dd}$, $\mathrm{J}=15.8,6.8 \mathrm{~Hz}, 1 \mathrm{H}), 3.98(\mathrm{~s}, 3 \mathrm{H}), 3.93(\mathrm{~s}, 3 \mathrm{H}), 2.27-2.18(\mathrm{~m}, 1 \mathrm{H}), 1.86(\mathrm{~d}, \mathrm{~J}=11.7 \mathrm{~Hz}$, $2 \mathrm{H}), 1.82-1.75(\mathrm{~m}, 2 \mathrm{H}), 1.69(\mathrm{~d}, \mathrm{~J}=12.7 \mathrm{~Hz}, 1 \mathrm{H}), 1.40-1.30(\mathrm{~m}, 2 \mathrm{H}), 1.27-1.18(\mathrm{~m}, 3 \mathrm{H})$. ${ }^{13} \mathrm{C} \mathrm{NMR}\left(126 \mathrm{MHz}, \mathrm{CDCl}_{3}\right) \delta 172.5,152.7,147.4,138.7,136.0,126.4,118.6,113.5,109.4$, $56.0,55.9,41.2,32.9,26.2,26.0$.

HRMS (ESI-TOF) m/z: [M + Na $]^{+}$Calcd for $\mathrm{C}_{17} \mathrm{H}_{22} \mathrm{O}_{4} \mathrm{Na} 313.1416$; Found 313.1413.

(E)-2-(2-Cyclohexylvinyl)-4,5-dimethylbenzoic acid (3ra).<smiles>Cc1cc(C=CC2CCCCC2)c(C(=O)O)cc1C</smiles>

White solid; eluent (hexane, 3\% ethyl acetate, and 1\% $\mathrm{HCOOH}$ ). The reaction scale is $50 \mathrm{mg}$, $61 \mathrm{mg}$ of product was isolated and yield is $77 \%$.

${ }^{1} \mathrm{H}$ NMR $\left(500 \mathrm{MHz}, \mathrm{CDCl}_{3}\right) \delta 7.78(\mathrm{~s}, 1 \mathrm{H}), 7.33(\mathrm{~s}, 1 \mathrm{H}), 7.20(\mathrm{~d}, \mathrm{~J}=15.8 \mathrm{~Hz}, 1 \mathrm{H}), 6.05(\mathrm{dd}, \mathrm{J}$ $=15.8,6.9 \mathrm{~Hz}, 1 \mathrm{H}), 2.31(\mathrm{~s}, 3 \mathrm{H}), 2.27(\mathrm{~s}, 3 \mathrm{H}), 2.22-2.16(\mathrm{~m}, 1 \mathrm{H}), 1.84(\mathrm{~d}, \mathrm{~J}=12.1 \mathrm{~Hz}, 2 \mathrm{H})$, $1.80-1.74(\mathrm{~m}, 2 \mathrm{H}), 1.68(\mathrm{~d}, \mathrm{~J}=12.7 \mathrm{~Hz}, 1 \mathrm{H}), 1.33(\mathrm{dd}, \mathrm{J}=25.0,12.5 \mathrm{~Hz}, 2 \mathrm{H}), 1.23(\mathrm{dd}, \mathrm{J}=$ 21.6, $9.0 \mathrm{~Hz}, 3 \mathrm{H})$.

${ }^{13} \mathrm{C} \mathrm{NMR}\left(126 \mathrm{MHz}, \mathrm{CDCl}_{3}\right) \delta 172.2,142.2,138.9,138.5,135.1,132.3,128.5,126.1,124.1$, $41.2,32.9,26.2,26.0,20.0,19.2$.

HRMS (ESI-TOF) m/z: [M + Na $]^{+}$Calcd for $\mathrm{C}_{17} \mathrm{H}_{22} \mathrm{O}_{2} \mathrm{Na} 281.1517$; Found 281.1523. 
(E)-3-(2-Cyclohexylvinyl)-2-naphthoic acid (3sa).<smiles>O=C(O)c1cc2ccccc2cc1/C=C/C1CCCCC1</smiles>

White solid; eluent (hexane, $4 \%$ ethyl acetate, and $1 \% \mathrm{HCOOH}$ ). The reaction scale is $50 \mathrm{mg}$, $50 \mathrm{mg}$ of product was isolated and yield is $62 \%$.

${ }^{1} \mathrm{H}$ NMR (500 MHz, $\left.\mathrm{CDCl}_{3}\right) \delta 8.61(\mathrm{~s}, 1 \mathrm{H}), 7.95(\mathrm{~s}, 1 \mathrm{H}), 7.90(\mathrm{~d}, \mathrm{~J}=7.9 \mathrm{~Hz}, 1 \mathrm{H}), 7.84(\mathrm{~d}, \mathrm{~J}=$ $8.2 \mathrm{~Hz}, 1 \mathrm{H}), 7.59-7.55$ (m, 1H), $7.51-7.46$ (m, 1H), 7.29 (s, 1H), 6.17 (dd, J = 15.7, 6.8 $\mathrm{Hz}, 1 \mathrm{H}), 2.25$ (ddd, J = 9.9, 8.8, 5.2 Hz, 1H), 1.95 - 1.87 (m, 2H), $1.85-1.77(\mathrm{~m}, 2 \mathrm{H}), 1.73-$ $1.68(\mathrm{~m}, 1 \mathrm{H}), 1.42-1.32(\mathrm{~m}, 2 \mathrm{H}), 1.27$ (ddd, J = 17.1, 10.6, $3.7 \mathrm{~Hz}, 3 \mathrm{H})$.

${ }^{13} \mathrm{C}$ NMR $\left(126 \mathrm{MHz}, \mathrm{CDCl}_{3}\right) \delta 172.1,139.4,136.7,135.5,132.9,131.3,128.9,128.6,127.5$, $126.6,126.2,126.2,125.7,41.2,32.9,26.2,26.0$.

HRMS (ESI-TOF) m/z: [M + Na $]^{+}$Calcd for $\mathrm{C}_{19} \mathrm{H}_{20} \mathrm{O}_{2} \mathrm{Na} 303.1361$; Found 303.1367.

6-(Hex-1-en-2-yl)-2,3-dimethoxybenzoic acid (4gb).<smiles>C=C(CCCC)c1ccc(OC)c(OC)c1C(=O)O</smiles>

Pale yellow liquid; eluent (hexane, 6\% ethyl acetate, and 1\% $\mathrm{HCOOH}$ ). The reaction scale is $50 \mathrm{mg}, 61 \mathrm{mg}$ of product was isolated and yield is $85 \%$.

${ }^{1} \mathrm{H}$ NMR $\left(500 \mathrm{MHz}, \mathrm{CDCl}_{3}\right) \delta 8.03(\mathrm{~d}, \mathrm{~J}=8.8 \mathrm{~Hz}, 1 \mathrm{H}), 7.28(\mathrm{~s}, 1 \mathrm{H}), 7.01(\mathrm{~d}, \mathrm{~J}=2.7 \mathrm{~Hz}, 1 \mathrm{H})$, $6.80(\mathrm{dd}, \mathrm{J}=8.7,2.6 \mathrm{~Hz}, 1 \mathrm{H}), 6.11(\mathrm{~d}, \mathrm{~J}=16.0 \mathrm{~Hz}, 1 \mathrm{H}), 3.89$ (s, 3H), 1.15 (s, 9H).

${ }^{13} \mathrm{C} \mathrm{NMR}\left(126 \mathrm{MHz}, \mathrm{CDCl}_{3}\right) \delta 170.9,151.4,148.3,145.9,134.6,132.2,124.2,114.0,113.6$, $61.8,56.0,37.1,30.1,22.3,13.9$.

HRMS (ESI-TOF) m/z: [M + Na $]^{+}$Calcd for $\mathrm{C}_{15} \mathrm{H}_{20} \mathrm{O}_{4} \mathrm{Na}$ 287.1259; Found287.1265. 


\section{2,3-Dimethoxy-6-(oct-1-en-2-yl)benzoic acid (4gc).}<smiles>C=C(CCCCCC)c1ccc(OC)c(OC)c1C(=O)O</smiles>

Pale yellow liquid; eluent (hexane, $5 \%$ ethyl acetate, and $1 \% \mathrm{HCOOH}$ ). The reaction scale is $50 \mathrm{mg}, 55 \mathrm{mg}$ of product was isolated and yield is $69 \%$.

${ }^{1} \mathrm{H}$ NMR $\left(500 \mathrm{MHz}, \mathrm{CDCl}_{3}\right) \delta 6.95(\mathrm{~d}, \mathrm{~J}=3.5 \mathrm{~Hz}, 2 \mathrm{H}), 5.09(\mathrm{q}, \mathrm{J}=1.5 \mathrm{~Hz}, 1 \mathrm{H}), 5.03(\mathrm{~d}, \mathrm{~J}=$ $1.6 \mathrm{~Hz}, 1 \mathrm{H}), 3.93(\mathrm{~s}, 3 \mathrm{H}), 3.89(\mathrm{~s}, 3 \mathrm{H}), 2.41-2.33(\mathrm{~m}, 2 \mathrm{H}), 1.45-1.36(\mathrm{~m}, 3 \mathrm{H}), 1.29$ (ddt, J = 7.3, 6.1, 2.2 Hz, 4H), $1.24(\mathrm{t}, \mathrm{J}=3.6 \mathrm{~Hz}, 2 \mathrm{H}), 0.86(\mathrm{t}, \mathrm{J}=7.0 \mathrm{~Hz}, 3 \mathrm{H})$.

${ }^{13} \mathrm{C} \mathrm{NMR}\left(126 \mathrm{MHz}, \mathrm{CDCl}_{3}\right) \delta 172.6,151.4,148.1,145.9,134.3,132.6,124.0,114.1,113.5$, $61.7,56.0,37.4,31.7,28.9,27.9,22.6,14.1$.

HRMS (ESI-TOF) m/z: [M + Na] $]^{+}$Calcd for $\mathrm{C}_{17} \mathrm{H}_{24} \mathrm{O}_{4} \mathrm{Na} 315.1572$; Found 315.1570.

\section{6-(Dec-1-en-2-yl)-2,3-dimethoxybenzoic acid (4gd).}<smiles>C=C(CCCCCCCC)c1ccc(OC)c(OC)c1C(=O)O</smiles>

Pale yellow liquid; eluent (hexane, $5 \%$ ethyl acetate, and $1 \% \mathrm{HCOOH}$ ). The reaction scale is $50 \mathrm{mg}, 50 \mathrm{mg}$ of product was isolated and yield is $58 \%$.

${ }^{1} \mathrm{H}$ NMR $\left(500 \mathrm{MHz}, \mathrm{CDCl}_{3}\right) \delta 6.95(\mathrm{~d}, \mathrm{~J}=3.5 \mathrm{~Hz}, 2 \mathrm{H}), 5.09(\mathrm{~s}, 1 \mathrm{H}), 5.02(\mathrm{~s}, 1 \mathrm{H}), 3.93(\mathrm{~s}, 3 \mathrm{H})$, $3.89(\mathrm{~s}, 3 \mathrm{H}), 2.36(\mathrm{t}, \mathrm{J}=7.7 \mathrm{~Hz}, 2 \mathrm{H}), 1.40(\mathrm{t}, \mathrm{J}=7.0 \mathrm{~Hz}, 2 \mathrm{H}), 1.25(\mathrm{~d}, \mathrm{~J}=10.3 \mathrm{~Hz}, 10 \mathrm{H}), 0.86$ $(\mathrm{t}, \mathrm{J}=6.9 \mathrm{~Hz}, 3 \mathrm{H})$.

${ }^{13} \mathrm{C}$ NMR $\left(126 \mathrm{MHz}, \mathrm{CDCl}_{3}\right) \delta 172.0,151.4,148.2,145.9,134.4,132.6,124.1,114.1,113.5$, $61.7,56.0,37.4,31.8,29.4,29.3,29.3,28.0,22.6,14.1$.

HRMS (ESI-TOF) m/z: [M + Na $]^{+}$Calcd for $\mathrm{C}_{19} \mathrm{H}_{28} \mathrm{O}_{4} \mathrm{Na} 343.1885$; Found 343.1886. 
(E)-2-(Hex-2-en-1-yl)-3,6-dimethoxybenzoic acid (5ib).<smiles>CCC/C=C/Cc1c(OC)ccc(OC)c1C(=O)O</smiles>

Pale yellow liquid; eluent (hexane, 5\% ethyl acetate, and $1 \% \mathrm{HCOOH}$ ). The reaction scale is $50 \mathrm{mg}, 52 \mathrm{mg}$ of product was isolated and yield is $73 \%$.

${ }^{1} \mathrm{H}$ NMR $\left(500 \mathrm{MHz}, \mathrm{CDCl}_{3}\right) \delta 6.80(\mathrm{~d}, \mathrm{~J}=8.9 \mathrm{~Hz}, 1 \mathrm{H}), 6.70(\mathrm{~d}, \mathrm{~J}=8.9 \mathrm{~Hz}, 1 \mathrm{H}), 5.41(\mathrm{dt}, \mathrm{J}=$ 14.6, 6.2 Hz, 2H), $3.76(\mathrm{~d}, \mathrm{~J}=5.6 \mathrm{~Hz}, 3 \mathrm{H}), 3.72(\mathrm{~s}, 3 \mathrm{H}), 3.36(\mathrm{~d}, \mathrm{~J}=5.7 \mathrm{~Hz}, 2 \mathrm{H}), 1.84$ (t, J = $7.0 \mathrm{~Hz}, 2 \mathrm{H}), 1.26$ (t, J = 7.4 Hz, 2H), 0.77 (t, J = 7.3 Hz, 3H).

${ }^{13} \mathrm{C} \mathrm{NMR}\left(126 \mathrm{MHz}, \mathrm{CDCl}_{3}\right) \delta 171.3,151.8,150.2,131.6,130.9,130.3,127.3,112.9,109.6$, $56.5,56.3,34.6,30.6,22.5,13.6$.

HRMS (ESI-TOF) m/z: [M + Na $]^{+}$Calcd for $\mathrm{C}_{15} \mathrm{H}_{20} \mathrm{O}_{4} \mathrm{Na} 287.1259$; Found 287.1257.

(E)-6-(3,3-Dimethylbut-1-en-1-yl)-2,3-dimethoxybenzoic acid (3ge).<smiles>COc1ccc(/C=C/C(C)(C)C)c(C(=O)O)c1OC</smiles>

White solid; eluent (hexane, $1 \%$ ethyl acetate, and $1 \% \mathrm{HCOOH}$ ). The reaction scale is $50 \mathrm{mg}$, $48 \mathrm{mg}$ of product was isolated and yield is $67 \%$.

${ }^{1} \mathrm{H}$ NMR $\left(500 \mathrm{MHz}, \mathrm{CDCl}_{3}\right) \delta 7.24(\mathrm{~d}, \mathrm{~J}=8.6 \mathrm{~Hz}, 1 \mathrm{H}), 6.96(\mathrm{~d}, \mathrm{~J}=8.6 \mathrm{~Hz}, 1 \mathrm{H}), 6.48(\mathrm{~d}, \mathrm{~J}=$ $16.0 \mathrm{~Hz}, 1 \mathrm{H}), 6.11(\mathrm{~d}, \mathrm{~J}=16.0 \mathrm{~Hz}, 1 \mathrm{H}), 3.92$ (s, 3H), 3.89 (s, 3H), 1.10 (s, 9H).

${ }^{13} \mathrm{C} \mathrm{NMR}\left(126 \mathrm{MHz}, \mathrm{CDCl}_{3}\right) \delta 171.3,151.2,146.1,143.7,129.8,126.1,122.1,121.1,114.4$, $61.8,56.0,33.5,29.5$.

HRMS (ESI-TOF) m/z: [M + Na $]^{+}$Calcd for $\mathrm{C}_{15} \mathrm{H}_{20} \mathrm{O}_{4} \mathrm{Na} 287.1259$; Found 287.1265. 
(E)-2-(3,3-Dimethylbut-1-en-1-yl)-4-methoxybenzoic acid (3je).<smiles>COc1ccc(C(=O)O)c(/C=C/C(C)(C)C)c1</smiles>

White solid; eluent (hexane, $1 \%$ ethyl acetate, and $1 \% \mathrm{HCOOH}$ ). The reaction scale is $50 \mathrm{mg}$, $26 \mathrm{mg}$ of product was isolated and yield is $34 \%$.

${ }^{1} \mathrm{H}$ NMR $\left(500 \mathrm{MHz}, \mathrm{CDCl}_{3}\right) \delta 8.03(\mathrm{~d}, \mathrm{~J}=8.8 \mathrm{~Hz}, 1 \mathrm{H}), 7.28(\mathrm{~s}, 1 \mathrm{H}), 7.01(\mathrm{~d}, \mathrm{~J}=2.7 \mathrm{~Hz}, 1 \mathrm{H})$, $6.80(\mathrm{dd}, \mathrm{J}=8.7,2.6 \mathrm{~Hz}, 1 \mathrm{H}), 6.11(\mathrm{~d}, \mathrm{~J}=16.0 \mathrm{~Hz}, 1 \mathrm{H}), 3.89$ (s, 3H), 1.15 (s, 9H).

${ }^{13} \mathrm{C}$ NMR $\left(126 \mathrm{MHz}, \mathrm{CDCl}_{3}\right) \delta 171.6,163.0,144.7,143.8,133.8,124.4,119.3,112.56,111.9$, $55.4,33.7,29.7,29.5,29.5$.

HRMS (ESI-TOF) m/z: [M + Na $]^{+}$Calcd for $\mathrm{C}_{14} \mathrm{H}_{18} \mathrm{O}_{3} \mathrm{Na} 257.1154$; Found 257.1156.

\section{2,6-Bis((E)-3,3-dimethylbut-1-en-1-yl)-4-methoxybenzoic acid (3je').}<smiles>COc1cc(/C=C/C(C)(C)C)c(C(=O)O)c(/C=C/C(C)(C)C)c1</smiles>

White solid; eluent (hexane, $1 \%$ ethyl acetate, and $1 \% \mathrm{HCOOH}$ ). The reaction scale is $50 \mathrm{mg}$, $30 \mathrm{mg}$ of product was isolated and yield is $29 \%$.

${ }^{1} \mathrm{H}$ NMR $\left(500 \mathrm{MHz}, \mathrm{CDCl}_{3}\right) \delta 6.89(\mathrm{~s}, 2 \mathrm{H}), 6.56(\mathrm{~d}, \mathrm{~J}=15.9 \mathrm{~Hz}, 2 \mathrm{H}), 6.16(\mathrm{~d}, \mathrm{~J}=15.9 \mathrm{~Hz}$, $2 \mathrm{H}), 3.87(\mathrm{~s}, 3 \mathrm{H}), 1.10(\mathrm{~s}, 18 \mathrm{H})$.

${ }^{13} \mathrm{C}$ NMR $\left(126 \mathrm{MHz}, \mathrm{CDCl}_{3}\right) \delta 173.6,160.5,145.1,138.9,122.6,122.4,109.9,55.4,33.6$, 29.4 .

HRMS (ESI-TOF) m/z: [M + Na $]^{+}$Calcd for $\mathrm{C}_{20} \mathrm{H}_{28} \mathrm{O}_{3} \mathrm{Na} 339.1936$; Found 339.1935. 
6-Cinnamyl-2,3-dimethoxybenzoic acid (5gf).<smiles>COc1ccc(C/C=C/c2ccccc2)c(C(=O)O)c1OC</smiles>

Pale yellow solid; eluent (hexane, $6 \%$ ethyl acetate, and $1 \% \mathrm{HCOOH}$ ). The reaction scale is $50 \mathrm{mg}, 70 \mathrm{mg}$ of product was isolated and yield is $87 \%$.

${ }^{1} \mathrm{H}$ NMR (500 MHz, $\left.\mathrm{CDCl}_{3}\right) \delta 7.33(\mathrm{~d}, \mathrm{~J}=7.6 \mathrm{~Hz}, 2 \mathrm{H}), 7.26(\mathrm{t}, \mathrm{J}=7.5 \mathrm{~Hz}, 2 \mathrm{H}), 7.18(\mathrm{~d}, \mathrm{~J}=$ $7.1 \mathrm{~Hz}, 1 \mathrm{H}), 7.00(\mathrm{~d}, \mathrm{~J}=8.4 \mathrm{~Hz}, 1 \mathrm{H}), 6.93(\mathrm{~d}, \mathrm{~J}=8.5 \mathrm{~Hz}, 1 \mathrm{H}), 6.43(\mathrm{~d}, \mathrm{~J}=15.8 \mathrm{~Hz}, 1 \mathrm{H}), 6.31$ $(\mathrm{dt}, \mathrm{J}=15.6,6.7 \mathrm{~Hz}, 1 \mathrm{H}), 3.92(\mathrm{~s}, 3 \mathrm{H}), 3.86(\mathrm{~s}, 3 \mathrm{H}), 3.59$ (d, J = 6.8 Hz, 2H).

${ }^{13} \mathrm{C} \mathrm{NMR}\left(126 \mathrm{MHz}, \mathrm{CDCl}_{3}\right) \delta 171.4,150.9,146.4,137.4,131.3,130.8,129.4,128.6,128.6$, $128.4,128.2,127.0,126.1,125.5,114.3,61.8,56.0,36.3$.

HRMS (ESI-TOF) m/z: [M + Na] $]^{+}$Calcd for $\mathrm{C}_{18} \mathrm{H}_{18} \mathrm{O}_{4} \mathrm{Na} 321.1103$; Found 321.1104.

6-(5-Hydroxypent-1-en-2-yl)-2,3-dimethoxybenzoic acid/(E)-6-(5-Hydroxypent-2-en-1yl)-2,3-dimethoxybenzoic acid (4gg).<smiles>C=C(CCCO)c1ccc(OC)c(OC)c1C(=O)O</smiles><smiles>COc1ccc(C/C=C/CCO)c(C(=O)O)c1OC</smiles>

Pale yellow liquid; eluent (hexane, $20 \%$ ethyl acetate, and 1\% $\mathrm{HCOOH}$ ). The reaction scale is $50 \mathrm{mg}, 48 \mathrm{mg}$ of product was isolated and yield is $66 \%$.

${ }^{1} \mathrm{H}$ NMR $\left(500 \mathrm{MHz}, \mathrm{CDCl}_{3}\right) \delta 8.05(\mathrm{~d}, \mathrm{~J}=10.1 \mathrm{~Hz}, 1 \mathrm{H}), 8.03(\mathrm{~s}, 1 \mathrm{H}), 6.94(\mathrm{~d}, \mathrm{~J}=7.2 \mathrm{~Hz}, 4 \mathrm{H})$, $6.53(\mathrm{~d}, \mathrm{~J}=15.8 \mathrm{~Hz}, 0.7 \mathrm{H}), 5.61-5.54(\mathrm{~m}, 0.7 \mathrm{H}), 5.50-5.43(\mathrm{~m}, 0.7 \mathrm{H}), 5.11(\mathrm{~s}, 1 \mathrm{H}), 5.06(\mathrm{~s}$, $1 \mathrm{H}), 4.16(\mathrm{dt}, \mathrm{J}=16.3,6.8 \mathrm{~Hz}, 4 \mathrm{H}), 3.92(\mathrm{q}, \mathrm{J}=3.7,2.7 \mathrm{~Hz}, 6 \mathrm{H}), 3.90-3.86(\mathrm{~m}, 6.7 \mathrm{H}), 3.40$ $(\mathrm{d}, \mathrm{J}=6.5 \mathrm{~Hz}, 1 \mathrm{H}), 2.42(\mathrm{t}, \mathrm{J}=7.6 \mathrm{~Hz}, 2 \mathrm{H}), 2.29-2.20(\mathrm{~m}, 1.3 \mathrm{H}), 2.10(\mathrm{~d}, \mathrm{~J}=7.2 \mathrm{~Hz}, 1 \mathrm{H})$, $1.71(\mathrm{dp}, \mathrm{J}=27.9,7.3,6.8 \mathrm{~Hz}, 4 \mathrm{H}), 1.55(\mathrm{~s}, 0.7 \mathrm{H}), 1.50(\mathrm{q}, \mathrm{J}=7.8 \mathrm{~Hz}, 2.4 \mathrm{H})$.

${ }^{13} \mathrm{C} \mathrm{NMR}\left(126 \mathrm{MHz}, \mathrm{CDCl}_{3}\right) \delta 171.4,170.9,161.3,151.6,150.9,146.3,145.9,133.2,131.6$, $130.9,130.8,130.7,127.5,127.4,126.6,125.4,125.2,125.1,124.8,123.9,123.9,123.8,122.0$, $117.4,115.2,114.8,114.2,114.2,113.5,113.4,63.4,61.7,55.9,36.0,33.5,31.7,30.7,29.6$, 26.7, 26.6 .

HRMS (ESI-TOF) m/z: [M + Na $]^{+}$Calcd for $\mathrm{C}_{14} \mathrm{H}_{18} \mathrm{O}_{5} \mathrm{Na} 289.1052$; Found 289.1047. 
6-(6-Hydroxyhex-1-en-2-yl)-2,3-dimethoxybenzoic acid/(E)-6-(6-Hydroxyhex-2-en-1-yl)2,3-dimethoxybenzoic acid (4gh).<smiles>C=C(CCCCO)c1ccc(OC)c(OC)c1C(=O)O</smiles>

Pale yellow liquid; eluent (hexane, $20 \%$ ethyl acetate, and $1 \% \mathrm{HCOOH}$ ). The reaction scale is $50 \mathrm{mg}, 54 \mathrm{mg}$ of product was isolated and yield is $70 \%$.

${ }^{1} \mathrm{H}$ NMR $\left(500 \mathrm{MHz}, \mathrm{CDCl}_{3}\right) \delta 8.06(\mathrm{~d}, \mathrm{~J}=10.0 \mathrm{~Hz}, 1 \mathrm{H}), 8.03(\mathrm{~s}, 1 \mathrm{H}), 6.94(\mathrm{~d}, \mathrm{~J}=7.4 \mathrm{~Hz}, 4 \mathrm{H})$, $6.53(\mathrm{~d}, \mathrm{~J}=15.8 \mathrm{~Hz}, 1 \mathrm{H}), 5.57(\mathrm{dt}, \mathrm{J}=14.7,6.7 \mathrm{~Hz}, 1 \mathrm{H}), 5.46(\mathrm{dt}, \mathrm{J}=15.0,6.7 \mathrm{~Hz}, 1 \mathrm{H}), 5.11$ (s, 1H), 5.06 (s, 1H), 4.16 (dt, J = 16.4, $6.8 \mathrm{~Hz}, 4 \mathrm{H}), 3.92$ (p, J = 4.4 Hz, 7H), $3.90-3.85$ (m, $6 \mathrm{H}), 3.40(\mathrm{~d}, \mathrm{~J}=6.6 \mathrm{~Hz}, 1 \mathrm{H}), 2.42(\mathrm{t}, \mathrm{J}=7.6 \mathrm{~Hz}, 2 \mathrm{H}), 2.23(\mathrm{q}, \mathrm{J}=7.5 \mathrm{~Hz}, 1 \mathrm{H}), 2.10(\mathrm{q}, \mathrm{J}=7.1$ $\mathrm{Hz}, 1 \mathrm{H}), 1.71(\mathrm{dp}, \mathrm{J}=27.9,7.3,6.7 \mathrm{~Hz}, 4 \mathrm{H}), 1.55(\mathrm{~s}, 1 \mathrm{H}), 1.50(\mathrm{q}, \mathrm{J}=7.8 \mathrm{~Hz}, 2 \mathrm{H})$.

${ }^{13} \mathrm{C}$ NMR $\left(126 \mathrm{MHz}, \mathrm{CDCl}_{3}\right) \delta 171.7,171.2,161.5,161.4,161.4,151.5,151.3,150.8,150.8$, 147.2, 146.3, 145.9, 145.8, 133.6, 132.8, 131.7, 131.4, 131.1, 131.0, 130.4, 129.5, 129.3, 129.0, $128.8,127.4,126.7,126.3,125.5,125.3,125.0,123.9,121.9,114.8,114.7,114.2,114.2,114.1$, $113.5,113.4,63.9,63.9,63.5,61.7,56.0,55.9,36.9,36.8,36.8,36.0,32.5,31.6,30.5,29.6$, $28.5,28.1,27.9,27.9,27.9,27.8,27.8,25.8,25.4,24.0,23.7,23.4$.

HRMS (ESI-TOF) m/z: [M + Na $]^{+}$Calcd for $\mathrm{C}_{15} \mathrm{H}_{20} \mathrm{O}_{5} \mathrm{Na} 303.1208$; Found 303.1211.

6-(10-Hydroxydec-1-en-2-yl)-2,3-dimethoxybenzoic acid/(E)-6-(10-Hydroxydec-2-en-1yl)-2,3-dimethoxybenzoic acid(4gi).<smiles>C=C(CCCCCCCCO)c1ccc(OC)c(OC)c1C(=O)O</smiles><smiles>COc1ccc(C/C=C/CCCCCCCO)c(C(=O)O)c1OC</smiles>

Pale yellow liquid; eluent (hexane, 20\% ethyl acetate, and 1\% $\mathrm{HCOOH}$ ). The reaction scale is $50 \mathrm{mg}, 78 \mathrm{mg}$ of product was isolated and yield is $85 \%$.

${ }^{1} \mathrm{H}$ NMR $\left(500 \mathrm{MHz}, \mathrm{CDCl}_{3}\right) \delta 8.05(\mathrm{~d}, \mathrm{~J}=4.0 \mathrm{~Hz}, 1.7 \mathrm{H}), 7.69(\mathrm{dd}, \mathrm{J}=7.6,2.0 \mathrm{~Hz}, 0.8 \mathrm{H}), 7.26$ $-7.14(\mathrm{~m}, 2.4 \mathrm{H}), 6.98-6.90(\mathrm{~m}, 4 \mathrm{H}), 6.52-6.44(\mathrm{~m}, 0.7 \mathrm{H}), 6.08(\mathrm{dq}, \mathrm{J}=16.0,8.9,7.9 \mathrm{~Hz}$, $0.6 \mathrm{H}), 5.54-5.46(\mathrm{~m}, 1 \mathrm{H}), 5.09(\mathrm{~s}, 1 \mathrm{H}), 5.03(\mathrm{~s}, 1 \mathrm{H}), 4.15(\mathrm{dt}, \mathrm{J}=10.2,6.5 \mathrm{~Hz}, 3.7 \mathrm{H}), 3.95-$ $3.90(\mathrm{~m}, 8.7 \mathrm{H}), 3.90-3.85(\mathrm{~m}, 6.9 \mathrm{H}), 3.66(\mathrm{dt}, \mathrm{J}=12.9,6.5 \mathrm{~Hz}, 0.8 \mathrm{H}), 3.38(\mathrm{~d}, \mathrm{~J}=5.6 \mathrm{~Hz}$, $0.8 \mathrm{H}), 2.37(\mathrm{t}, \mathrm{J}=7.5 \mathrm{~Hz}, 2 \mathrm{H}), 2.22-2.15(\mathrm{~m}, 1 \mathrm{H}), 2.00(\mathrm{q}, \mathrm{J}=6.6 \mathrm{~Hz}, 1 \mathrm{H}), 1.69-1.60(\mathrm{~m}$, $3.8 \mathrm{H}), 1.56(\mathrm{dt}, \mathrm{J}=15.0,7.7 \mathrm{~Hz}, 1.2 \mathrm{H}), 1.33$ (ddt, J = 33.9, 18.8, 9.1 Hz, 24.5H).

${ }^{13} \mathrm{C} \mathrm{NMR}\left(126 \mathrm{MHz}, \mathrm{CDCl}_{3}\right) \delta 171.8,171.5,171.1,166.3,152.2,151.4,151.2,150.7,148.4$, 148.0, 147.8, 146.2, 145.9, 145.8, 145.7, 134.1, 133.9, 132.7, 132.7, 132.3, 132.1, 131.4, 131.3, $129.1,128.0,127.4,125.9,125.2,124.8,124.0,123.9,123.7,122.4,121.8,117.4,114.3,114.2$, 
114.13, 114.1, 114.0, 113.4, 113.3, 64.1, 62.9, 62.1, 61.7, 61.6, 56.1, 56.0, 55.9, 55.9, 37.3, 37.2 , 35.9, 33.0, 32.3, 32.2, 32.1, 29.6, 29.4, 29.3, 29.2 , 29.2, 29.2, 29.1, 29.1, 29.1, 29.0, 29.0, $28.9,28.9,28.9,28.8,28.8,28.7,28.6,28.4,28.4,28.4,27.8,27.5,25.7,25.7,25.7,25.6,25.43$, 25.3, 25.3.

HRMS (ESI-TOF) m/z: [M + Na $]^{+}$Calcd for $\mathrm{C}_{19} \mathrm{H}_{28} \mathrm{O}_{5} \mathrm{Na} 359.1834$; Found 359.1833.

2-(10-Hydroxydec-1-en-2-yl)-4-methoxybenzoic acid /(E)-2-(10-Hydroxydec-1-en-1-yl)4-methoxybenzoic acid (4ji).<smiles>C=C(CCCCCCCCO)c1cc(OC)ccc1C(=O)O</smiles><smiles>COc1ccc(C(=O)O)c(/C=C/CCCCCCCCO)c1</smiles>

Pale yellow liquid; eluent (hexane, $20 \%$ ethyl acetate, and 1\% $\mathrm{HCOOH}$ ). The reaction scale is $50 \mathrm{mg}, 40 \mathrm{mg}$ of product was isolated and yield is $40 \%$.

${ }^{1} \mathrm{H}$ NMR $\left(500 \mathrm{MHz}, \mathrm{CDCl}_{3}\right) \delta 8.08-8.04(\mathrm{~m}, 1.7 \mathrm{H}), 8.01(\mathrm{dt}, \mathrm{J}=8.8,5.2 \mathrm{~Hz}, 1.7 \mathrm{H}), 7.34-$ $7.28(\mathrm{~m}, 0.7 \mathrm{H}), 7.02(\mathrm{~d}, \mathrm{~J}=2.6 \mathrm{~Hz}, 0.6 \mathrm{H}), 6.84(\mathrm{dt}, \mathrm{J}=9.0,2.3 \mathrm{~Hz}, 0.6 \mathrm{H}), 6.80(\mathrm{dd}, \mathrm{J}=8.8$, $2.6 \mathrm{~Hz}, 0.7 \mathrm{H}), 6.70(\mathrm{~d}, \mathrm{~J}=2.6 \mathrm{~Hz}, 1 \mathrm{H}), 6.12(\mathrm{dt}, \mathrm{J}=15.7,6.9 \mathrm{~Hz}, 0.7 \mathrm{H}), 5.09(\mathrm{~d}, \mathrm{~J}=1.7 \mathrm{~Hz}$, $1 \mathrm{H}), 4.91(\mathrm{~d}, \mathrm{~J}=1.8 \mathrm{~Hz}, 1 \mathrm{H}), 4.15(\mathrm{dt}, \mathrm{J}=10.2,6.7 \mathrm{~Hz}, 4 \mathrm{H}), 3.88(\mathrm{~s}, 1.7 \mathrm{H}), 3.87(\mathrm{~s}, 3 \mathrm{H}), 3.68$ $-3.62(\mathrm{~m}, 0.7 \mathrm{H}), 2.38(\mathrm{t}, \mathrm{J}=7.7 \mathrm{~Hz}, 2 \mathrm{H}), 2.26(\mathrm{qd}, \mathrm{J}=7.0,1.5 \mathrm{~Hz}, 1.7 \mathrm{H}), 1.70-1.61(\mathrm{~m}, 4 \mathrm{H})$, $1.50(\mathrm{t}, \mathrm{J}=7.3 \mathrm{~Hz}, 1.7 \mathrm{H}), 1.42-1.27(\mathrm{~m}, 19.3 \mathrm{H})$.

${ }^{13} \mathrm{C}$ NMR $\left(126 \mathrm{MHz}, \mathrm{CDCl}_{3}\right) \delta 171.5,171.0,163.0,162.7,161.3,161.2,161.2,151.7,148.6$, 143.4, 139.1, 134.2, 133.8, 133.5, 129.1, 119.9, 119.1, 116.1, 116.0, 114.2, 112.6, 112.0, 112.0, 64.1, 64.1, 63.0, 55.4, 55.4, 37.4, 33.7, 33.0, 29.3, 29.2, 29.2, 29.2, 29.1, 29.0, 28.5, 28.4, 28.04, $25.8,25.7$.

HRMS (ESI-TOF) m/z: [M + Na $]^{+}$Calcd for $\mathrm{C}_{18} \mathrm{H}_{26} \mathrm{O}_{4} \mathrm{Na} 329.1729$; Found 329.1737.

(E)-2-(5-(Benzoyloxy)pent-1-en-1-yl)-6-methoxybenzoic acid (3aj).<smiles>COc1cccc(/C=C/CCCOC(=O)c2ccccc2)c1C(=O)O</smiles>

Pale yellow solid; eluent (hexane, $15 \%$ ethyl acetate, and $1 \% \mathrm{HCOOH}$ ). The reaction scale is $50 \mathrm{mg}, 42 \mathrm{mg}$ of product was isolated and yield is $38 \%$.

${ }^{1} \mathrm{H}$ NMR $\left(500 \mathrm{MHz}, \mathrm{CDCl}_{3}\right) \delta 8.07-8.01(\mathrm{~m}, 2 \mathrm{H}), 7.57-7.51(\mathrm{~m}, 1 \mathrm{H}), 7.42(\mathrm{t}, J=7.6 \mathrm{~Hz}$, 2H), $7.32(\mathrm{t}, J=8.0 \mathrm{~Hz}, 1 \mathrm{H}), 7.12(\mathrm{~d}, J=7.9 \mathrm{~Hz}, 1 \mathrm{H}), 6.84(\mathrm{~d}, J=8.3 \mathrm{~Hz}, 1 \mathrm{H}), 6.73(\mathrm{~d}, J=$ $15.7 \mathrm{~Hz}, 1 \mathrm{H}), 6.21(\mathrm{dt}, J=15.7,6.9 \mathrm{~Hz}, 1 \mathrm{H}), 4.38(\mathrm{t}, J=6.6 \mathrm{~Hz}, 2 \mathrm{H}), 3.89(\mathrm{~s}, 3 \mathrm{H}), 2.43-2.36$ $(\mathrm{m}, 2 \mathrm{H}), 1.97(\mathrm{q}, J=7.0 \mathrm{~Hz}, 2 \mathrm{H})$. 
${ }^{13} \mathrm{C} \mathrm{NMR}\left(126 \mathrm{MHz}, \mathrm{CDCl}_{3}\right) \delta 169.5,166.7,156.8,138.2,133.0,133.0,132.9,132.8,131.1$, $130.3,129.6,129.6,129.5,128.3,128.1,119.0,109.6,64.4,56.2,29.6,28.2$.

HRMS (ESI-TOF) m/z: [M + Na $]^{+}$Calcd for $\mathrm{C}_{20} \mathrm{H}_{20} \mathrm{O}_{5} \mathrm{Na} 363.1208$; Found 363.1205.

(E)-2-(4-(1,1-Dioxido-3-oxobenzo[d]isothiazol-2(3H)-yl)but-1-en-1-yl)-6-methoxybenzoic acid/ 2-(4-(1,1-Dioxido-3-oxobenzo[d]isothiazol-2(3H)-yl)but-1-en-2-yl)-6methoxybenzoic acid (3ak).<smiles>COc1cccc(/C=C/CCN2C(=O)c3ccccc3S2(=O)=O)c1C(=O)O</smiles><smiles>C=C(CCN1C(=O)c2ccccc2S1(=O)=O)c1cccc(OC)c1C(=O)O</smiles>

White solid; eluent (hexane, $20 \%$ ethyl acetate, and $1 \% \mathrm{HCOOH}$ ). The reaction scale is 50 $\mathrm{mg}, 45 \mathrm{mg}$ of product was isolated and yield is $55 \%$.

${ }^{1} \mathrm{H}$ NMR (500 MHz, CDCl3) $\delta 8.07-8.03(\mathrm{~m}, 1.63 \mathrm{H}), 7.91(\mathrm{~d}, \mathrm{~J}=7.6 \mathrm{~Hz}, 1.2 \mathrm{H}), 7.87-7.78$ $(\mathrm{m}, 2.5 \mathrm{H}), 7.32(\mathrm{t}, \mathrm{J}=8.1 \mathrm{~Hz}, 1 \mathrm{H}), 7.15(\mathrm{~d}, \mathrm{~J}=7.8 \mathrm{~Hz}, 1 \mathrm{H}), 6.83(\mathrm{~d}, \mathrm{~J}=8.3 \mathrm{~Hz}, 1 \mathrm{H}), 6.77(\mathrm{~d}$, $\mathrm{J}=15.6 \mathrm{~Hz}, 1 \mathrm{H}), 6.20(\mathrm{dt}, \mathrm{J}=15.0,7.1 \mathrm{~Hz}, 1 \mathrm{H}), 6.05(\mathrm{~m}, 0.3 \mathrm{H}), 5.69(\mathrm{~m}, 0.3 \mathrm{H}), 5.33(\mathrm{~s}, 0.1 \mathrm{H})$, $5.23(\mathrm{~s}, 0.1 \mathrm{H}), 4.36(\mathrm{~d}, 0.46 \mathrm{H}), 3.96-3.91(\mathrm{~m}, 2 \mathrm{H}), 3.88(\mathrm{~d}, \mathrm{~J}=4.7 \mathrm{~Hz}, 3.4 \mathrm{H}), 3.57(\mathrm{~d}, 0.5 \mathrm{H})$, $2.77(\mathrm{t}, \mathrm{J}=7.1 \mathrm{~Hz}, 2 \mathrm{H})$.

${ }^{13} \mathrm{C} \mathrm{NMR}\left(126 \mathrm{MHz}, \mathrm{CDCl}_{3}\right) \delta 169.9,163.1,159.0,156.6,139.6,137.7,137.6,137.5,134.7$, $134.7,134.3,134.3,131.2,131.1,130.9,130.3,130.3,129.1,129.0,127.4,127.3,125.2,125.1$, $124.3,122.6,121.0,120.9,120.9,120.8,120.7,119.0,117.4,110.4,109.9,109.4,56.21,56.2$, 40.7, 38.7, 36.7, 36.4, 32.1, 31.6.

HRMS (ESI-TOF) m/z: [M + Na $]^{+}$Calcd for $\mathrm{C}_{19} \mathrm{H}_{17} \mathrm{NSO}_{6} \mathrm{Na} 410.0674$; Found 410.0684. 
(E)-6-(4-(1,1-Dioxido-3-oxobenzo[d]isothiazol-2(3H)-yl)but-1-en-1-yl)-2,3-

dimethoxybenzoic acid/6-(4-(1,1-Dioxido-3-oxobenzo[d]isothiazol-2(3H)-yl)but-1-en-2yl)-2,3-dimethoxybenzoic acid (3gk).<smiles>C=C(CCN1C(=O)c2ccccc2S1(=O)=O)c1ccc(OC)c(OC)c1C(=O)O</smiles>

White solid; eluent (hexane, $20 \%$ ethyl acetate, and $1 \% \mathrm{HCOOH}$ ). The reaction scale is 50 $\mathrm{mg}, 70 \mathrm{mg}$ of product was isolated and yield is $62 \%$.

${ }^{1} \mathrm{H}$ NMR $\left(500 \mathrm{MHz}, \mathrm{CDCl}_{3}\right) \delta 8.04(\mathrm{t}, J=12.3 \mathrm{~Hz}, 2.8 \mathrm{H}), 7.91(\mathrm{~d}, J=7.3 \mathrm{~Hz}, 2.6 \mathrm{H}), 7.88-$ $7.75(\mathrm{~m}, 3.6 \mathrm{H}), 7.31-7.24(\mathrm{~m}, 1.5 \mathrm{H}), 7.01-6.90(\mathrm{~m}, 2.5 \mathrm{H}), 6.72-6.60(\mathrm{~m}, 1.3 \mathrm{H}), 6.07(\mathrm{tt}$, $J=15.9,7.7 \mathrm{~Hz}, 1.3 \mathrm{H}), 5.29(\mathrm{~s}, 0.4 \mathrm{H}), 5.2(\mathrm{~s}, 0.3 \mathrm{H}), 3.97-3.81(\mathrm{~m}, 14.7 \mathrm{H}), 2.75(\mathrm{~d}, J=7.3$ $\mathrm{Hz}, 2 \mathrm{H})$.

${ }^{13} \mathrm{C} \mathrm{NMR}\left(126 \mathrm{MHz}, \mathrm{CDCl}_{3}\right) \delta 170.1,163.4,158.9,158.7,158.5,151.8,151.5,150.9,146.2$, $145.9,145.8,143.2$, 139.2, 137.6, 137.4, 137.4, 134.7, 134.4, 134.3, 132.0, 129.8, 129.3, 129.3, $128.4,127.8,127.5,127.2$, 127.1, 127.0, 126.6, 125.4, 125.1, 125.1, 125.0, 123.9, 122.1, 120.8, $120.8,120.8,119.4,117.1,114.1,113.5,61.7,61.6,61.6,55.9,55.9,40.6,38.7,38.6,38.1$, $36.9,35.9,35.3$.

HRMS (ESI-TOF) m/z: [M + Na $]^{+}$Calcd for $\mathrm{C}_{20} \mathrm{H}_{19} \mathrm{NSO}_{7} \mathrm{Na} 440.0780$; Found 440.0794.

(E)-2-Methoxy-6-(4-((2-oxo-2H-chromen-4-yl)oxy)but-1-en-1-yl)benzoic acid (3al).<smiles>COc1cccc(/C=C/CCOc2cc(=O)oc3ccccc23)c1C(=O)O</smiles>

Pale yellow solid; eluent (hexane, $15 \%$ ethyl acetate, and $1 \% \mathrm{HCOOH}$ ). The reaction scale is $50 \mathrm{mg}, 54 \mathrm{mg}$ of product was isolated and yield is $45 \%$.

${ }^{1} \mathrm{H}$ NMR (500 MHz, DMSO) $\delta 7.81(\mathrm{dd}, \mathrm{J}=7.9,1.7 \mathrm{~Hz}, 1 \mathrm{H}), 7.66-7.63(\mathrm{~m}, 1 \mathrm{H}), 7.39-7.31$ $(\mathrm{m}, 3 \mathrm{H}), 7.21(\mathrm{~d}, \mathrm{~J}=7.9 \mathrm{~Hz}, 1 \mathrm{H}), 6.95(\mathrm{~d}, \mathrm{~J}=8.2 \mathrm{~Hz}, 1 \mathrm{H}), 6.54(\mathrm{~d}, \mathrm{~J}=15.6 \mathrm{~Hz}, 1 \mathrm{H}), 6.42(\mathrm{dt}$, $\mathrm{J}=15.7,6.9 \mathrm{~Hz}, 1 \mathrm{H}), 5.91(\mathrm{~s}, 1 \mathrm{H}), 4.31(\mathrm{t}, \mathrm{J}=6.1 \mathrm{~Hz}, 2 \mathrm{H}), 3.77(\mathrm{~s}, 3 \mathrm{H}), 2.79-2.70(\mathrm{~m}, 2 \mathrm{H})$.

13C NMR (126 MHz, DMSO) $\delta$ 169.2, 165.3, 162.1, 155.9, 153.2, 134.6, 133.2, 130.2, 129.5, $129.0,124.7,124.7,123.4,117.5,116.8,115.7,110.5,91.0,68.8,56.2,56.2,56.2,56.1,32.50$. HRMS (ESI-TOF) m/z: [M + Na $]^{+}$Calcd for $\mathrm{C}_{21} \mathrm{H}_{18} \mathrm{O}_{6} \mathrm{Na} 389.1001$; Found 389.0999. 
5-Methyl-2-((E)-4-(((8R,9S,13S,14S)-13-methyl-17-oxo-7,8,9,11,12,13,14,15,16,17decahydro-6H-cyclopenta[a]phenanthren-3-yl)oxy)but-1-en-1-yl)benzoic acid/5-Methyl2-(4-((8R,9S,13S,14S)-13-methyl-17-oxo-7,8,9,11,12,13,14,15,16,17-decahydro-6Hcyclopenta[a]phenanthren-3-yl)oxy)but-1-en-2-yl)benzoic acid (3nm).<smiles>C=C(CCOc1ccc2c(c1)CC[C@H]1[C@@H]3CCC(=O)[C@@]3(C)CC[C@@H]21)c1ccc(C)cc1C(=O)O</smiles>

White solid; eluent (hexane, $15 \%$ ethyl acetate, and $1 \% \mathrm{HCOOH}$ ). The reaction scale is 50 $\mathrm{mg}, 95 \mathrm{mg}$ of product was isolated and yield is $58 \%$.

${ }^{1} \mathrm{H}$ NMR $\left(500 \mathrm{MHz}, \mathrm{CDCl}_{3}\right) \delta 7.80(\mathrm{dd}, \mathrm{J}=15.4,1.9 \mathrm{~Hz}, 1.3 \mathrm{H}), 7.47(\mathrm{~d}, \mathrm{~J}=7.9 \mathrm{~Hz}, 1 \mathrm{H}), 7.38$ $-7.28(\mathrm{~m}, 2.8 \mathrm{H}), 7.21-7.14(\mathrm{~m}, 1.8 \mathrm{H}), 6.75(\mathrm{dd}, \mathrm{J}=8.6,2.8 \mathrm{~Hz}, 1 \mathrm{H}), 6.67(\mathrm{~d}, \mathrm{~J}=2.8 \mathrm{~Hz}$, $1 \mathrm{H}), 6.19(\mathrm{dt}, \mathrm{J}=15.8,6.9 \mathrm{~Hz}, 1 \mathrm{H}), 5.27(\mathrm{~s}, 0.3 \mathrm{H}), 5.05(\mathrm{~s}, 0.25 \mathrm{H}), 4.08(\mathrm{t}, \mathrm{J}=6.7 \mathrm{~Hz}, 2 \mathrm{H})$, $4.0(\mathrm{t}, \mathrm{J}=6.7 \mathrm{~Hz}, 0.5 \mathrm{H}), 2.88(\mathrm{dtd}, \mathrm{J}=20.8,12.1,10.6,6.2 \mathrm{~Hz}, 3.7 \mathrm{H}), 2.72(\mathrm{qd}, \mathrm{J}=6.7,1.5$ $\mathrm{Hz}, 2 \mathrm{H}), 2.53-2.46(\mathrm{~m}, 1.72 \mathrm{H}), 2.39$ (s, 1H), 2.37 (s, 3.7H), $2.29-2.20(\mathrm{~m}, 2 \mathrm{H}), 2.19-2.09$ $(\mathrm{m}, 2 \mathrm{H}), 2.08-1.91(\mathrm{~m}, 5.5 \mathrm{H}), 1.66-1.50(\mathrm{~m}, 5.2 \mathrm{H}), 1.50-1.40(\mathrm{~m}, 5.2 \mathrm{H}), 0.90(\mathrm{~s}, 3 \mathrm{H}), 0.89$ $(\mathrm{s}, 0.87 \mathrm{H})$.

${ }^{13} \mathrm{C}$ NMR $\left(126 \mathrm{MHz}, \mathrm{CDCl}_{3}\right) \delta 172.0,156.9,156.5,146.4,137.7,137.3,137.3,136.8,133.7$, 133.1, 132.2, 132.1, 131.6, 131.5, 130.7, 129.9, 128.6, 128.5, 127.5, 126.7, 126.3, 126.3, 115.8, $114.7,114.7,112.3,112.2,67.4,66.3,50.4,48.0,44.0,38.4,38.3,37.2,35.9,33.1,31.9,31.6$, $26.5,26.5,25.9,25.9,22.7,21.6,20.9,14.1013 .8$.

HRMS (ESI-TOF) m/z: [M + Na $]^{+}$Calcd for $\mathrm{C}_{30} \mathrm{H}_{34} \mathrm{O}_{4} \mathrm{Na}$ 481.2355; Found 481.2357.

(2Z,4E)-5-Cyclohexyl-2-methylpenta-2,4-dienoic acid (6aa).<smiles>C/C(=C/C=C/C1CCCCC1)C(=O)O</smiles>

White solid; eluent (hexane, $1 \%$ ethyl acetate, and $1 \% \mathrm{HCOOH}$ ). The reaction scale is $50 \mathrm{mg}$, $70 \mathrm{mg}$ of product was isolated and yield is $63 \%$.

${ }^{1} \mathrm{H}$ NMR $\left(500 \mathrm{MHz}, \mathrm{CDCl}_{3}\right) \delta 7.16(\mathrm{dd}, \mathrm{J}=15.3,11.2 \mathrm{~Hz}, 1 \mathrm{H}), 6.53(\mathrm{~d}, \mathrm{~J}=11.2 \mathrm{~Hz}, 1 \mathrm{H}), 5.90$ $(\mathrm{dd}, \mathrm{J}=15.3,7.2 \mathrm{~Hz}, 1 \mathrm{H}), 2.12(\mathrm{dt}, \mathrm{J}=7.4,3.6 \mathrm{~Hz}, 1 \mathrm{H}), 1.97$ (s, 3H), 1.74 (dp, J = 10.1, 3.9 $\mathrm{Hz}, 4 \mathrm{H}), 1.69-1.64(\mathrm{~m}, 1 \mathrm{H}), 1.32-1.27(\mathrm{~m}, 2 \mathrm{H}), 1.16(\mathrm{ddd}, \mathrm{J}=23.2,10.0,6.8 \mathrm{~Hz}, 3 \mathrm{H})$.

${ }^{13} \mathrm{C} \mathrm{NMR}\left(126 \mathrm{MHz}, \mathrm{CDCl}_{3}\right) \delta 173.5,149.3,144.1,125.4,122.8,41.2,32.5,26.0,25.8,20.4$. HRMS (ESI-TOF) m/z: Calcd for $\mathrm{C}_{12} \mathrm{H}_{18} \mathrm{O}_{2}$ 194.1307; Found 194.1156. 
(2Z,4E)-2-Methylnona-2,4-dienoic acid/(2Z,4Z)-2-methylnona-2,4-dienoic acid (6ab).<smiles>CCCC/C=C\C=C(\C)C(=O)O</smiles>

Colorless liquid; eluent (hexane, $1 \%$ ethyl acetate, and $1 \% \mathrm{HCOOH}$ ). The reaction scale is 50 $\mathrm{mg}, 30 \mathrm{mg}$ of product was isolated and yield is $31 \%$.

${ }^{1} \mathrm{H}$ NMR $\left(500 \mathrm{MHz}, \mathrm{CDCl}_{3}\right) \delta 7.17(\mathrm{dd}, \mathrm{J}=15.1,11.3 \mathrm{~Hz}, 1 \mathrm{H}), 7.05(\mathrm{~m}, 0.2 \mathrm{H}), 6.88(\mathrm{~d}, \mathrm{~J}=10$ $\mathrm{Hz}, 0.18 \mathrm{H}), 6.53(\mathrm{~d}, \mathrm{~J}=11.3 \mathrm{~Hz}, 1 \mathrm{H}), 5.97(\mathrm{dt}, \mathrm{J}=14.7,7.1 \mathrm{~Hz}, 1 \mathrm{H}), 5.7(\mathrm{~m}, 0.2 \mathrm{H}), 2.18$ (q, J $=7.3 \mathrm{~Hz}, 2 \mathrm{H}), 1.96(\mathrm{~s}, 3 \mathrm{H}), 1.40(\mathrm{ddd}, \mathrm{J}=12.0,9.1,5.6 \mathrm{~Hz}, 3 \mathrm{H}), 1.37-1.30(\mathrm{~m}, 3 \mathrm{H}), 0.93-$ $0.87(\mathrm{~m}, 4.7 \mathrm{H})$.

${ }^{13} \mathrm{C} \mathrm{NMR}\left(126 \mathrm{MHz}, \mathrm{CDCl}_{3}\right) \delta 173.1,143.8,143.7,139.5,137.0,127.8,127.7,125.2,122.6$, $32.7,31.1,22.3,22.3,20.4,13.9,13.9$.

HRMS (ESI-TOF) m/z: [M + H $]^{+}$Calcd for $\mathrm{C}_{10} \mathrm{H}_{17} \mathrm{O}_{4} \mathrm{H}$ 169.1229; Found 169.0832.

(2Z,4E)-2-Methylundeca-2,4-dienoic acid/(2Z,4Z)-2-Methylundeca-2,4-dienoic acid(6ac)<smiles>CCCCCC/C=C\C=C(\C)C(=O)O</smiles>

Colorless liquid; eluent (hexane, $1 \%$ ethyl acetate, and $1 \% \mathrm{HCOOH}$ ). The reaction scale is 50 $\mathrm{mg}, 50 \mathrm{mg}$ of product was isolated and yield is $45 \%$.

${ }^{1} \mathrm{H} \mathrm{NMR}\left(500 \mathrm{MHz}, \mathrm{CDCl}_{3}\right) \delta 7.10(\mathrm{dd}, \mathrm{J}=15.1,11.3 \mathrm{~Hz}, 1 \mathrm{H}), 6.46(\mathrm{~d}, \mathrm{~J}=11.3 \mathrm{~Hz}, 1 \mathrm{H}), 6.19$ (s, 0.4H), $5.90(\mathrm{dt}, \mathrm{J}=14.6,7.1 \mathrm{~Hz}, 1 \mathrm{H}), 4.94-4.85(\mathrm{~m}, 1 \mathrm{H}), 2.14-2.06(\mathrm{~m}, 3 \mathrm{H}), 1.93-1.91$ $(\mathrm{m}, 1.7 \mathrm{H}), 1.89(\mathrm{~s}, 3 \mathrm{H}), 1.36-1.32(\mathrm{~m}, 3.6 \mathrm{H}), 1.23(\mathrm{dd}, \mathrm{J}=11.8,6.7 \mathrm{~Hz}, 13.3 \mathrm{H}), 0.81(\mathrm{td}, \mathrm{J}=$ $6.9,3.1 \mathrm{~Hz}, 7.7 \mathrm{H})$.

${ }^{13} \mathrm{C}$ NMR $\left(126 \mathrm{MHz}, \mathrm{CDCl}_{3}\right) \delta 173.3,145.8,143.9,143.8,143.8,143.7,139.6,139.26,138.7$, 138.7, 137.0, 128.0, 127.8, 125.2, 123.4, 122.8, 122.6, 114.2, 114.0, 39.5, 38.7, 35.8, 33.8, $33.0,32.1,31.9,31.7,29.7,29.0,28.9,28.9,28.1,27.6,27.4,23.4,22.7,22.6,22.6,20.9,20.83$, $20.4,18.2,17.8,16.3,14.1$.

HRMS (ESI-TOF) m/z: [M + Na $]^{+}$Calcd for $\mathrm{C}_{12} \mathrm{H}_{20} \mathrm{O}_{2} \mathrm{Na} 219.1361$; Found 219.1358. 
(2Z,4E)-2-Methyltrideca-2,4-dienoic acid (6ad).<smiles>CCCCCCCC/C=C\C(C)C(=O)O</smiles>

Colorless liquid; eluent (hexane, $1 \%$ ethyl acetate, and $1 \% \mathrm{HCOOH}$ ). The reaction scale is 50 $\mathrm{mg}, 32 \mathrm{mg}$ of product was isolated and yield is $22 \%$.

${ }^{1} \mathrm{H}$ NMR $\left(500 \mathrm{MHz}, \mathrm{CDCl}_{3}\right) \delta 7.17(\mathrm{dd}, \mathrm{J}=15.1,11.3 \mathrm{~Hz}, 1 \mathrm{H}), 6.53(\mathrm{~d}, \mathrm{~J}=11.2 \mathrm{~Hz}, 1 \mathrm{H}), 5.97$ (dt, J = 14.7, 7.1 Hz, 1H), $2.17(\mathrm{q}, \mathrm{J}=7.3 \mathrm{~Hz}, 2 \mathrm{H}), 1.97(\mathrm{~s}, 3 \mathrm{H}), 1.41(\mathrm{p}, \mathrm{J}=7.4 \mathrm{~Hz}, 2 \mathrm{H}), 1.28$ $(\mathrm{dq}, \mathrm{J}=10.5,6.4,5.2 \mathrm{~Hz}, 12 \mathrm{H}), 0.88(\mathrm{t}, \mathrm{J}=6.8 \mathrm{~Hz}, 3 \mathrm{H})$.

${ }^{13} \mathrm{C}$ NMR $\left(126 \mathrm{MHz}, \mathrm{CDCl}_{3}\right) \delta 173.1,143.8,143.7,127.8,122.6,33.0,31.8,29.4,29.2,29.2$, 29.1, 22.6, 20.4, 14.1.

HRMS (ESI-TOF) m/z: [M + $\left.\mathrm{NH}_{4}\right]$ Calcd for $\mathrm{C}_{14} \mathrm{H}_{24} \mathrm{O}_{2} \mathrm{NH}_{4}$ 242.2120; Found 242.2124.

(2Z,4E)-2-Methyl-6-phenylhexa-2,4-dienoic dienoic acid(6af/6af').

$\operatorname{acid}(2 Z, 4 E)-2-M e t h y l-6-p h e n y l h e x a-2,4-$<smiles>C/C(=C/CC=Cc1ccccc1)C(=O)O</smiles>

Pale yellow liquid; eluent (hexane, $1 \%$ ethyl acetate, and $1 \% \mathrm{HCOOH}$ ). The reaction scale is $50 \mathrm{mg}, 62 \mathrm{mg}$ of product was isolated and yield is $53 \%$.

${ }^{1} \mathrm{H}$ NMR $\left(500 \mathrm{MHz}, \mathrm{CDCl}_{3}\right) \delta 7.32-7.26(\mathrm{~m}, 3.6 \mathrm{H}), 7.23-7.16(\mathrm{~m}, 4.3 \mathrm{H}), 6.59-6.50(\mathrm{~m}$, $1 \mathrm{H}), 6.26(\mathrm{~m}, 0.2 \mathrm{H}), 6.06(\mathrm{dt}, \mathrm{J}=14.9,7.3 \mathrm{~Hz}, 1 \mathrm{H}), 5.92(\mathrm{~m}, 0.2 \mathrm{H}), 3.62(\mathrm{~d}, 0.4 \mathrm{H}), 3.51(\mathrm{~d}, \mathrm{~J}=$ $7.3 \mathrm{~Hz}, 2 \mathrm{H}), 2.05(\mathrm{~d}, \mathrm{~J}=1.4 \mathrm{~Hz}, 1 \mathrm{H}), 2.00-1.96(\mathrm{~m}, 3 \mathrm{H})$.

${ }^{13} \mathrm{C}$ NMR $\left(126 \mathrm{MHz}, \mathrm{CDCl}_{3}\right) \delta 173.3,173.1,143.0,141.1,139.5,136.5,136.4,128.8,128.7$, $128.7,128.7,128.6,128.6,128.6,128.6,128.5,128.5,128.5,128.4,128.4,128.3,128.3,128.3$, $128.2,127.8,126.3,126.3,126.2,126.2,125.9,123.8,39.4,20.9,20.4$.

HRMS (ESI-TOF) m/z: [M + Na $]^{+}$Calcd for $\mathrm{C}_{13} \mathrm{H}_{14} \mathrm{O}_{2} \mathrm{Na} 225.0891$; Found 225.0887. 
(2Z,4E)-5-Cyclohexyl-2-(4-methoxyphenyl)penta-2,4-dienoic acid/(2Z,4Z)-5-Cyclohexyl2-(4-methoxyphenyl)penta-2,4-dienoic acid (6ga).<smiles>COc1ccc(C(=CC=CC2CCCCC2)C(=O)O)cc1</smiles>

Pale yellow liquid; eluent (hexane, $1 \%$ ethyl acetate, and $1 \% \mathrm{HCOOH}$ ). The reaction scale is $50 \mathrm{mg}, 55 \mathrm{mg}$ of product was isolated and yield is $69 \%$.

${ }^{1} \mathrm{H}$ NMR (500 MHz, $\left.\mathrm{CDCl}_{3}\right) \delta 7.38-7.34(\mathrm{~m}, 2 \mathrm{H}), 7.32(\mathrm{~d}, \mathrm{~J}=8.7 \mathrm{~Hz}, 0.8 \mathrm{H}), 7.03(\mathrm{dd}, \mathrm{J}=$ 12.0, 1.1 Hz, 1.2H), $6.90(\mathrm{~d}, \mathrm{~J}=8.7 \mathrm{~Hz}, 2.8 \mathrm{H}), 6.87(\mathrm{~d}, \mathrm{~J}=8.8 \mathrm{~Hz}, 1.2 \mathrm{H}), 6.85-6.78(\mathrm{~m}$, $1 \mathrm{H}), 6.72(\mathrm{~d}, 0.4 \mathrm{H}), 6.02-6.07(\mathrm{dd}, 0.5 \mathrm{H}), 5.71(\mathrm{ddd}, \mathrm{J}=11.0,9.7,1.2 \mathrm{~Hz}, 1 \mathrm{H}), 3.83(\mathrm{~s}, 3 \mathrm{H})$, $3.81(\mathrm{~s}, 1 \mathrm{H}), 2.57$ (ddt, J = 14.5, 10.9, $5.5 \mathrm{~Hz}, 1 \mathrm{H}), 1.75(\mathrm{ddq}, \mathrm{J}=20.0,6.8,4.8,3.3 \mathrm{~Hz}$, $4.5 \mathrm{H}), 1.70-1.63(\mathrm{~m}, 4.5 \mathrm{H}), 1.35-1.26(\mathrm{~m}, 5 \mathrm{H}), 1.20-1.09(\mathrm{~m}, 5.2 \mathrm{H})$.

${ }^{13} \mathrm{C}$ NMR $\left(126 \mathrm{MHz}, \mathrm{CDCl}_{3}\right) \delta 172.8,172.6,159.4,159.1,150.5,146.1,142.2,134.2,132.3$, $131.5,131.0,130.8,130.7,129.5,129.3,128.5,125.4,123.2,113.7,113.7,113.6,55.3,55.3$, $41.4,36.8,33.1,33.0,33.0,32.9,32.5,32.3,31.9,26.0,25.8,25.6$.

HRMS (ESI-TOF) m/z: [M + Na $]^{+}$Calcd for $\mathrm{C}_{18} \mathrm{H}_{22} \mathrm{O}_{3} \mathrm{Na} 309.1467$; Found 309.1474. 
${ }^{1} \mathrm{H}$ and ${ }^{13} \mathrm{C}$ NMR Spectra of Compound 3aa. $\left(\mathrm{CDCl}_{3}\right.$ solvent was used, $\left.500 \mathrm{NMR} \mathrm{MHz}\right)$
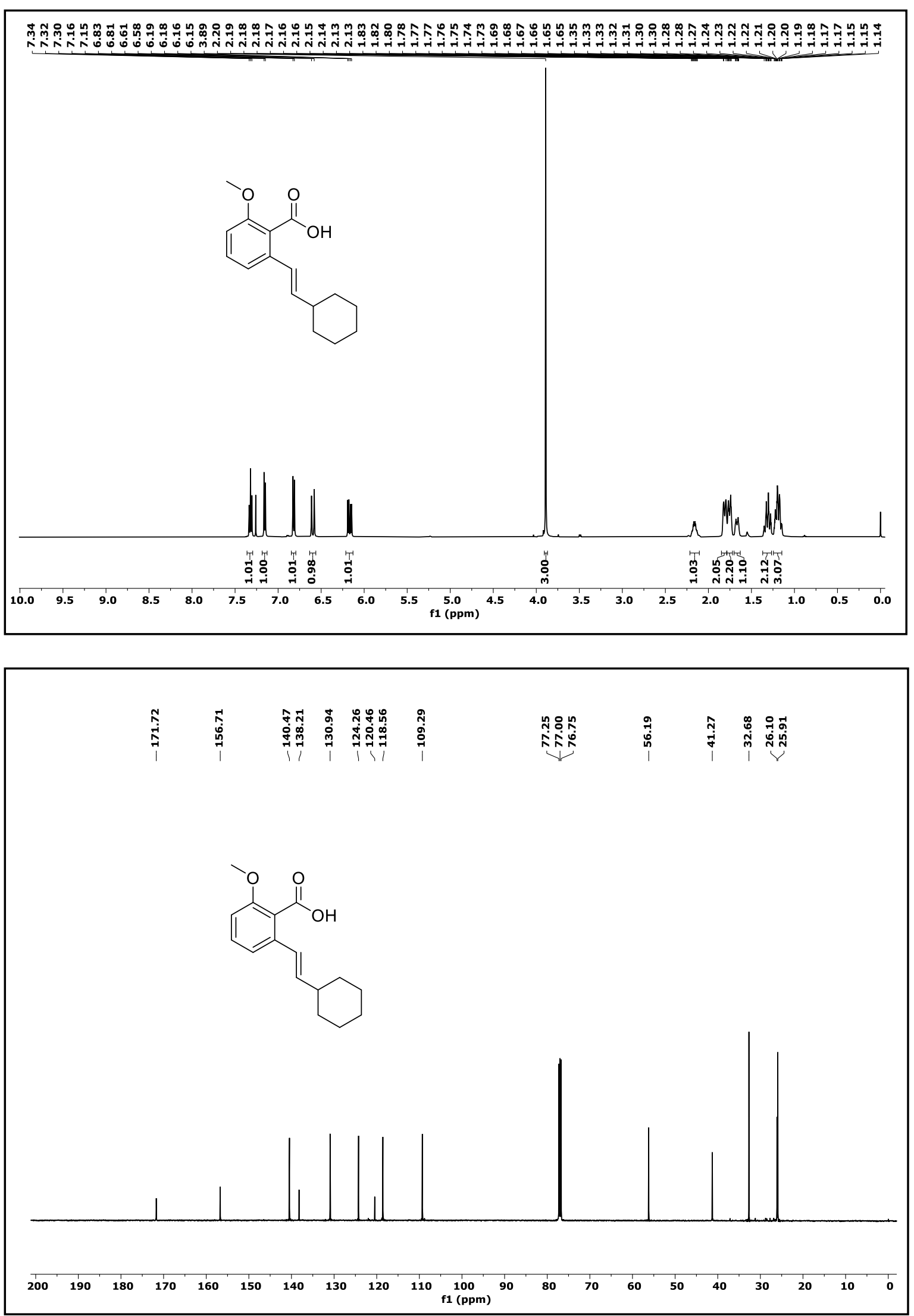
${ }^{1} \mathrm{H}$ and ${ }^{13} \mathrm{C}$ NMR Spectra of Compound 3ba. $\left(\mathrm{CDCl}_{3}\right.$ solvent was used, $\left.500 \mathrm{NMR} \mathrm{MHz}\right)$
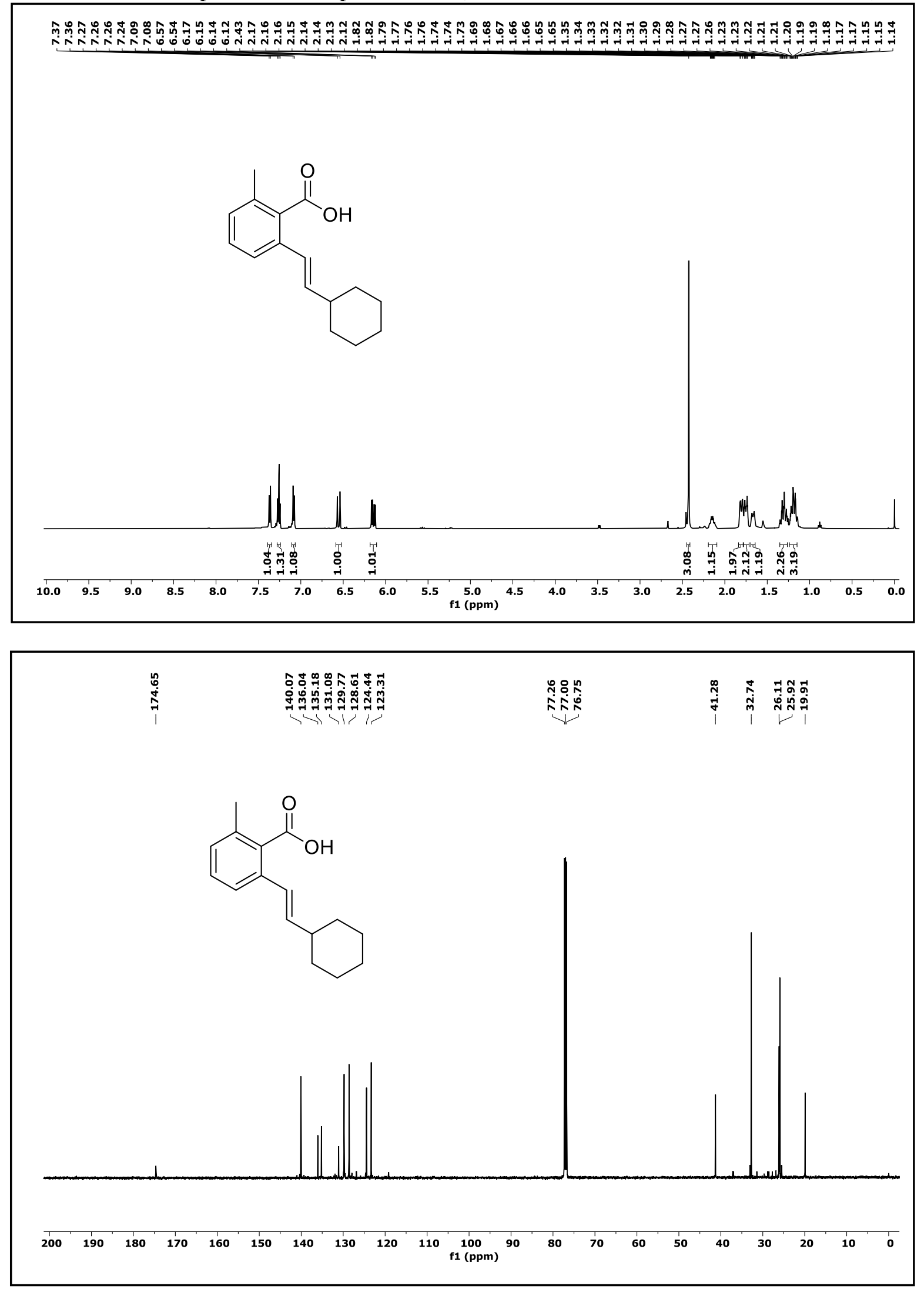
${ }^{1} \mathrm{H}$ and ${ }^{13} \mathrm{C}$ NMR Spectra of Compound 3ca. $\left(\mathrm{CDCl}_{3}\right.$ solvent was used, $\left.500 \mathrm{NMR} \mathrm{MHz}\right)$

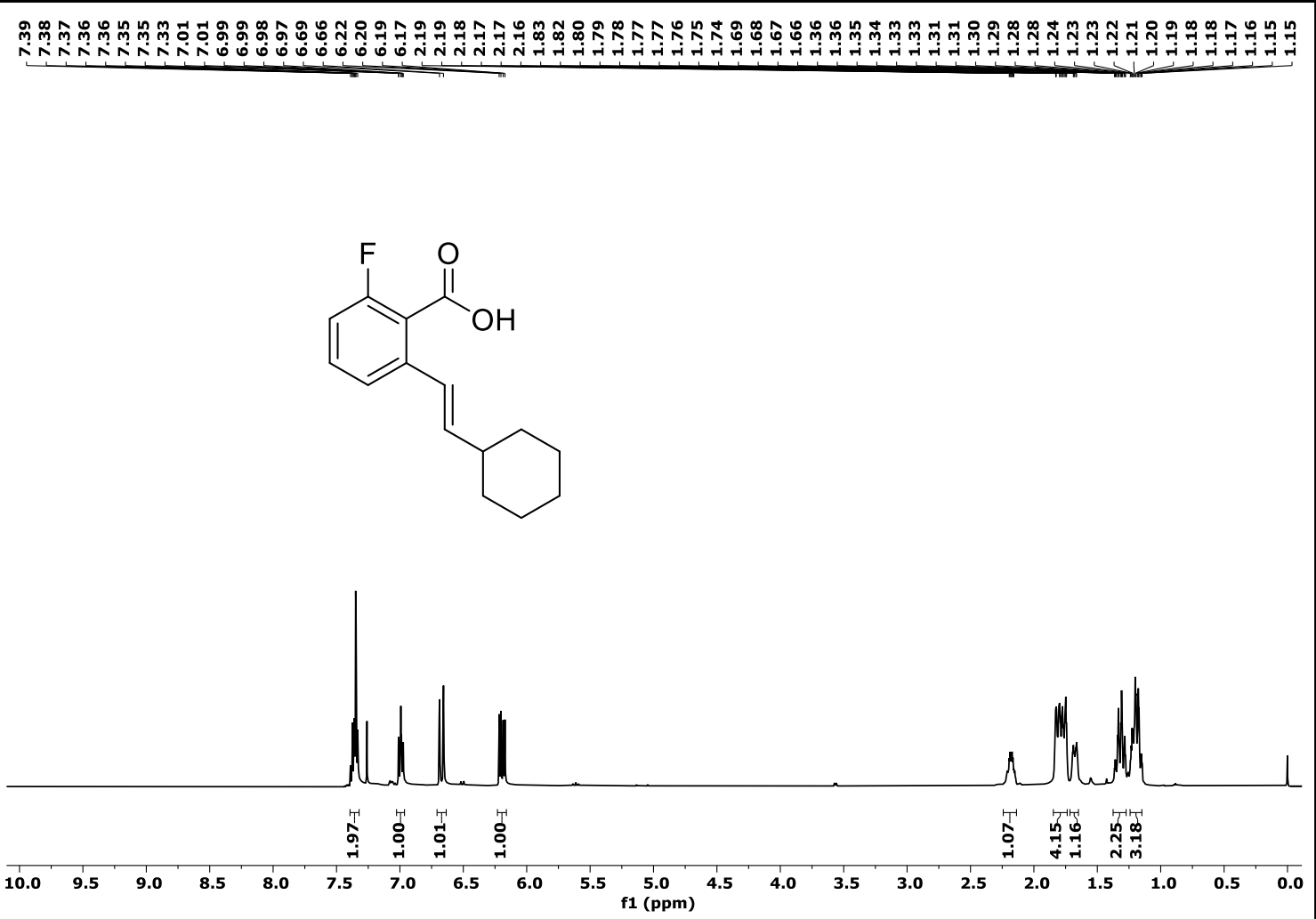

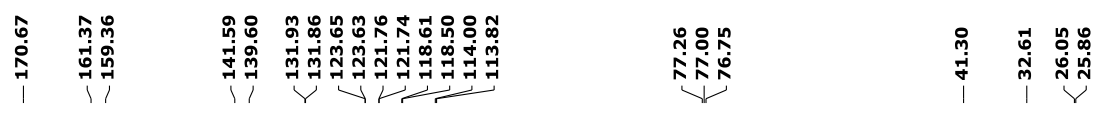

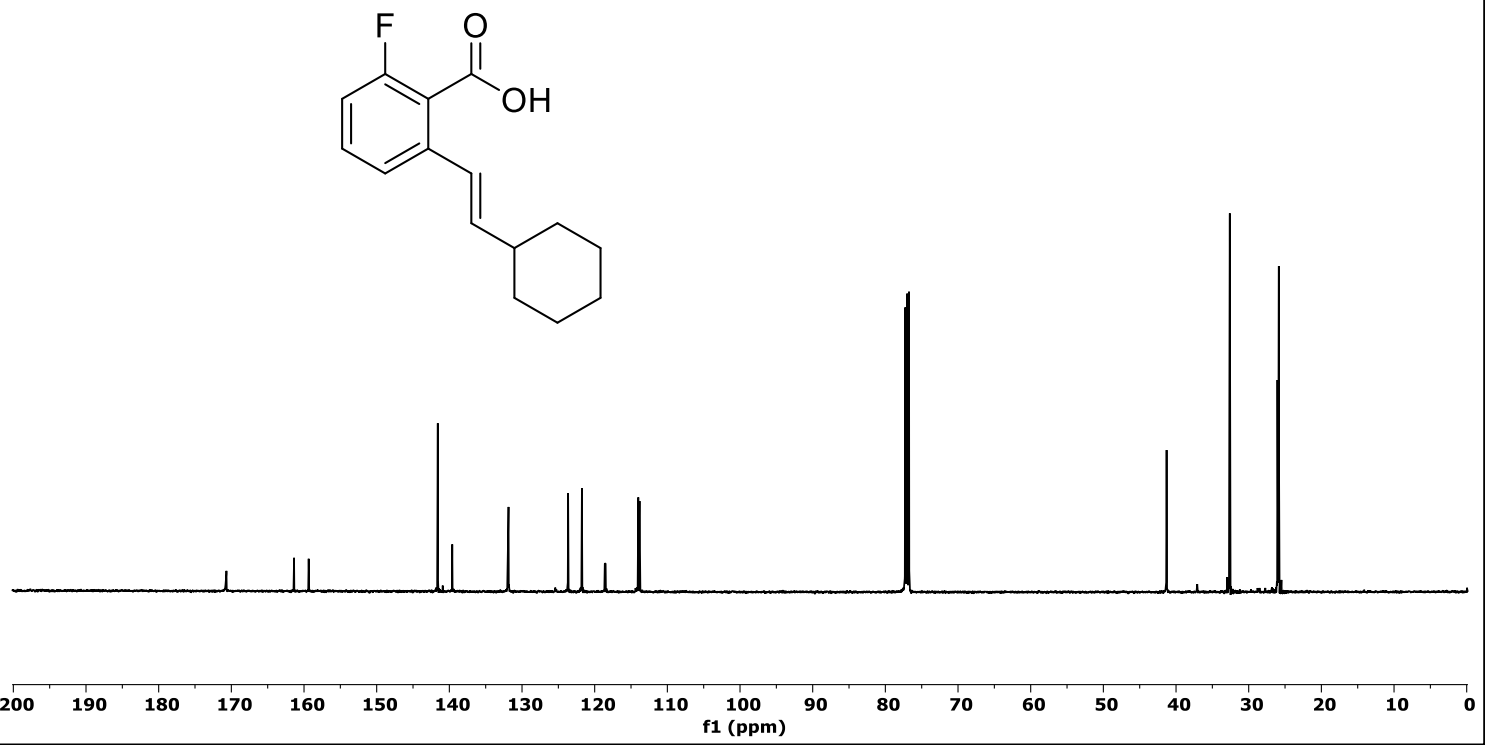


${ }^{1} \mathrm{H}$ and ${ }^{13} \mathrm{C}$ NMR Spectra of Compound 3da. (DMSO solvent was used, $500 \mathrm{NMR} \mathrm{MHz}$ ).
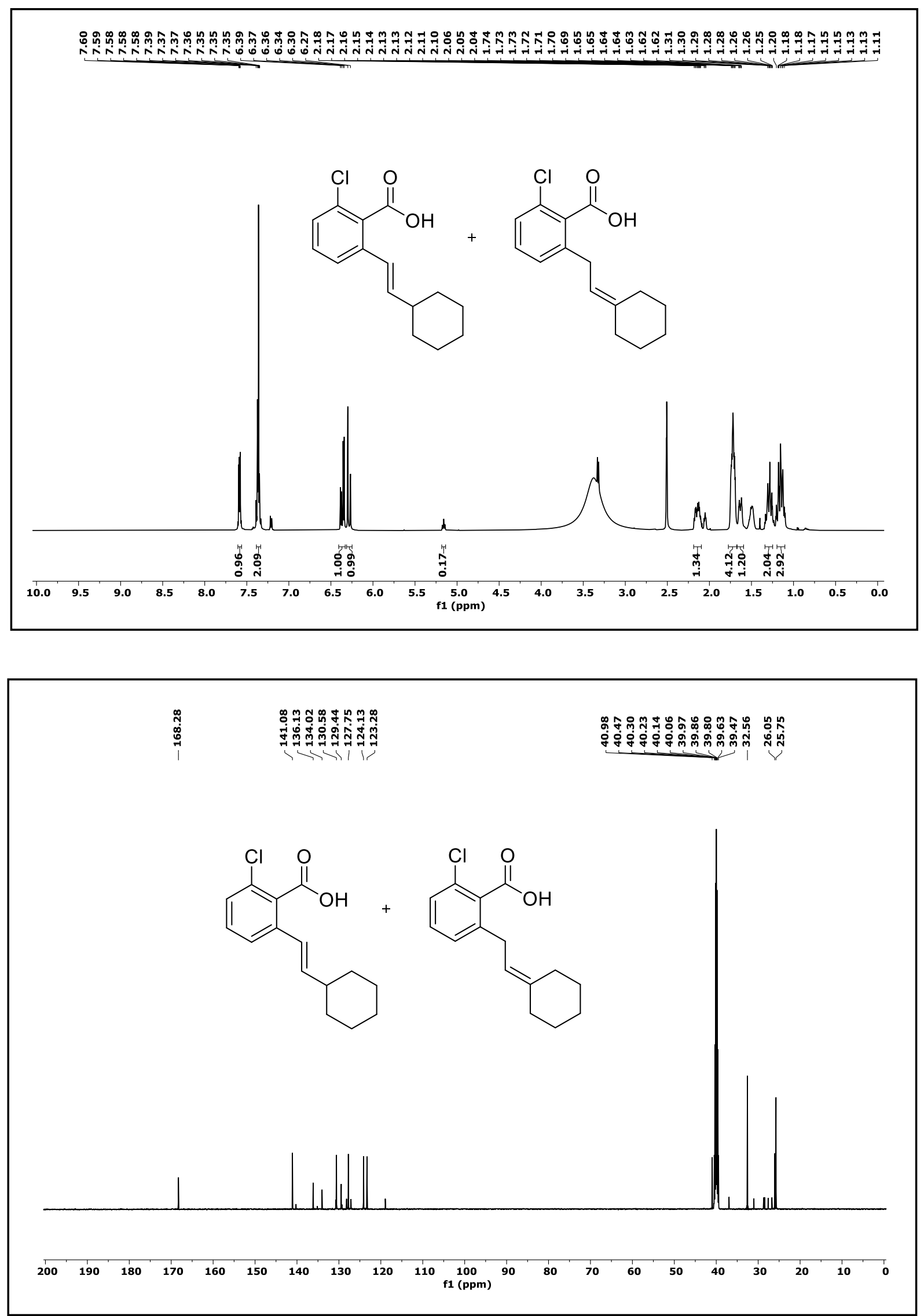
${ }^{1} \mathrm{H}$ and ${ }^{13} \mathrm{C}$ NMR Spectra of Compound 3ea. (DMSO solvent was used, $500 \mathrm{NMR} \mathrm{MHz}$ ).
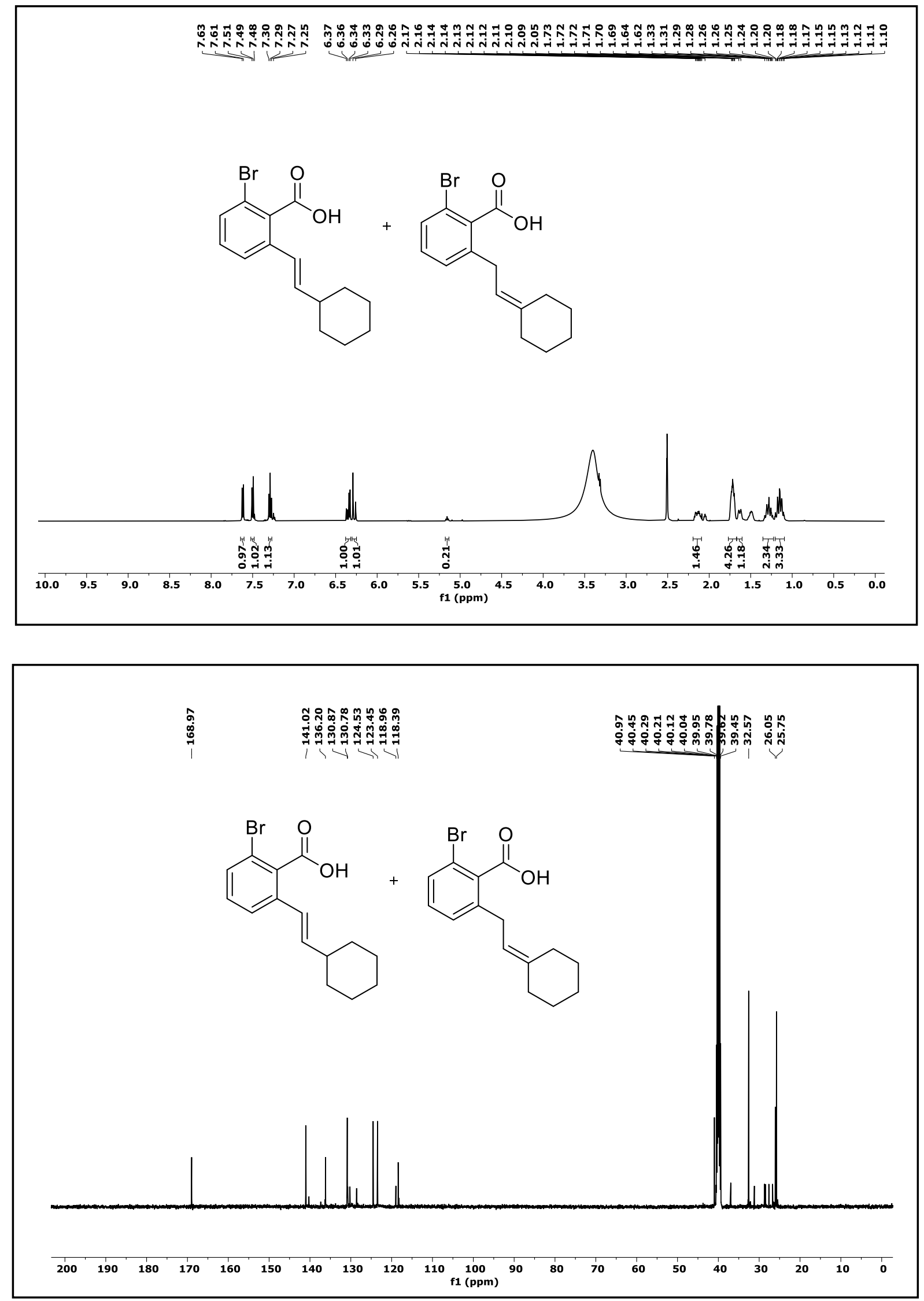
${ }^{1} \mathrm{H}$ and ${ }^{13} \mathrm{C}$ NMR Spectra of Compound 3fa. $\left(\mathrm{CDCl}_{3}\right.$ solvent was used, $\left.500 \mathrm{NMR} \mathrm{MHz}\right)$

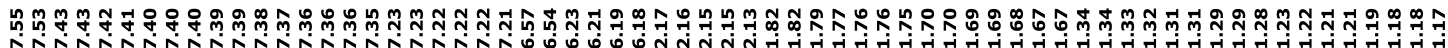<smiles>O=C(O)c1c(/C=C/C2CCCCC2)cccc1-c1ccccc1</smiles>
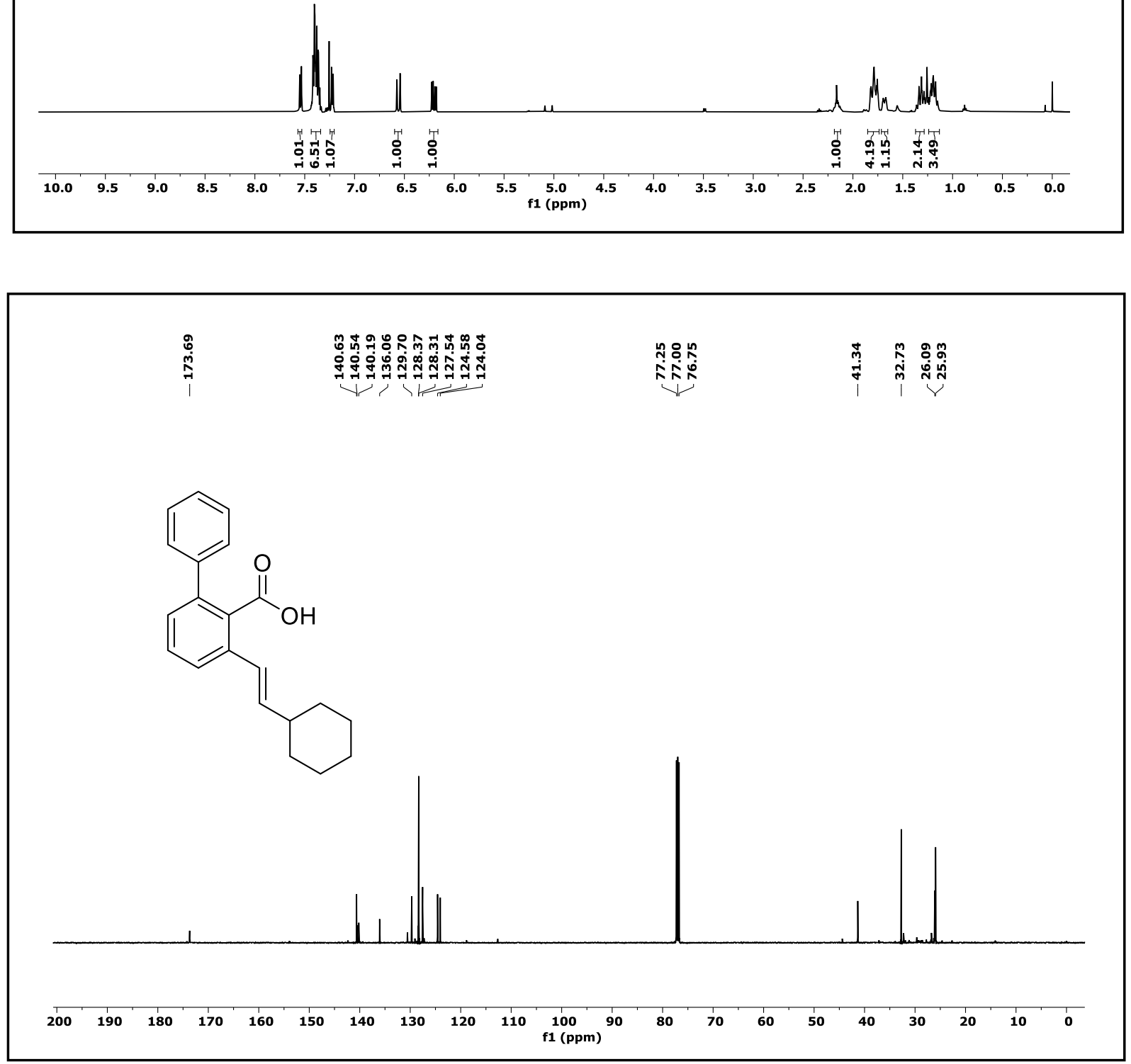
${ }^{1} \mathrm{H}$ and ${ }^{13} \mathrm{C}$ NMR Spectra of Compound 3ga. $\left(\mathrm{CDCl}_{3}\right.$ solvent was used, $\left.500 \mathrm{NMR} \mathrm{MHz}\right)$

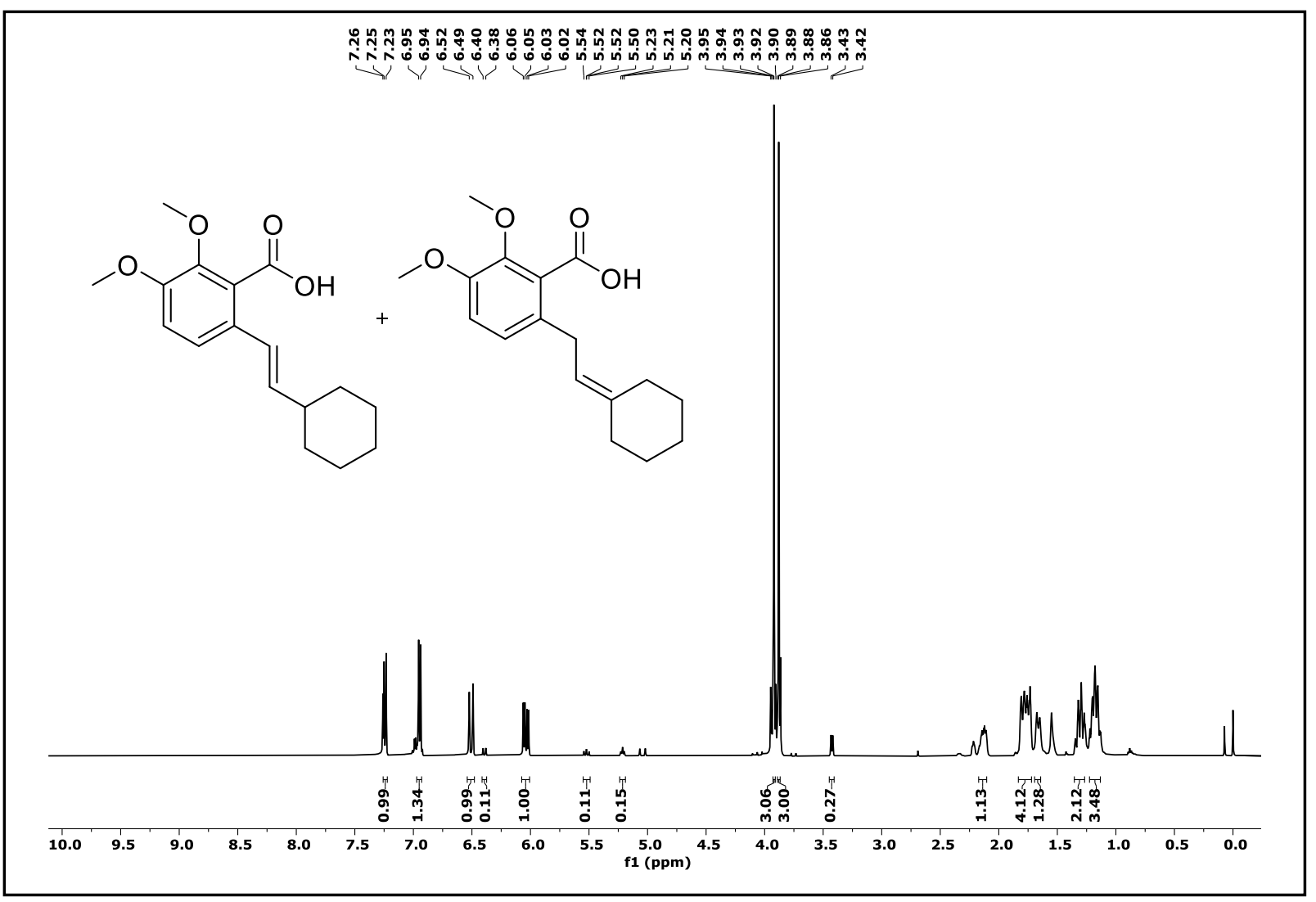

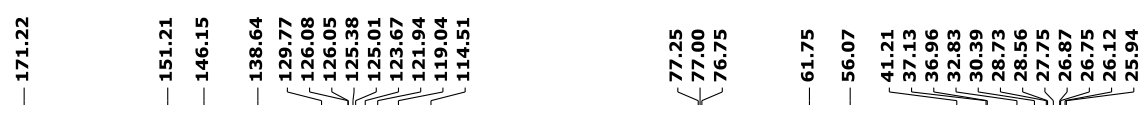<smiles>COc1ccc(C=CC2CCCCC2)c(C(=O)O)c1OC</smiles>

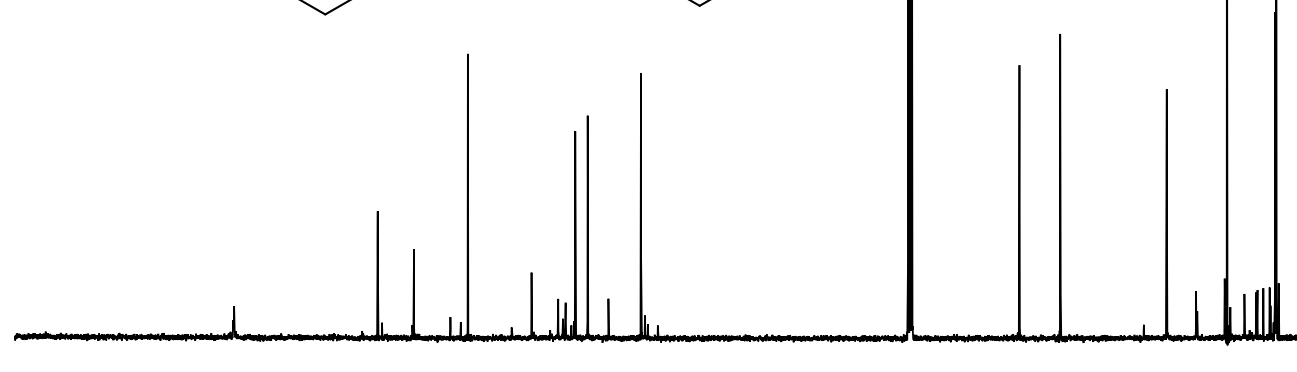

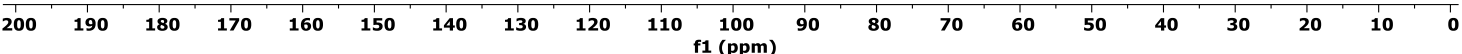


${ }^{1} \mathrm{H}$ and ${ }^{13} \mathrm{C}$ NMR Spectra of Compound 3ha. $\left(\mathrm{CDCl}_{3}\right.$ solvent was used, $\left.500 \mathrm{NMR} \mathrm{MHz}\right)$
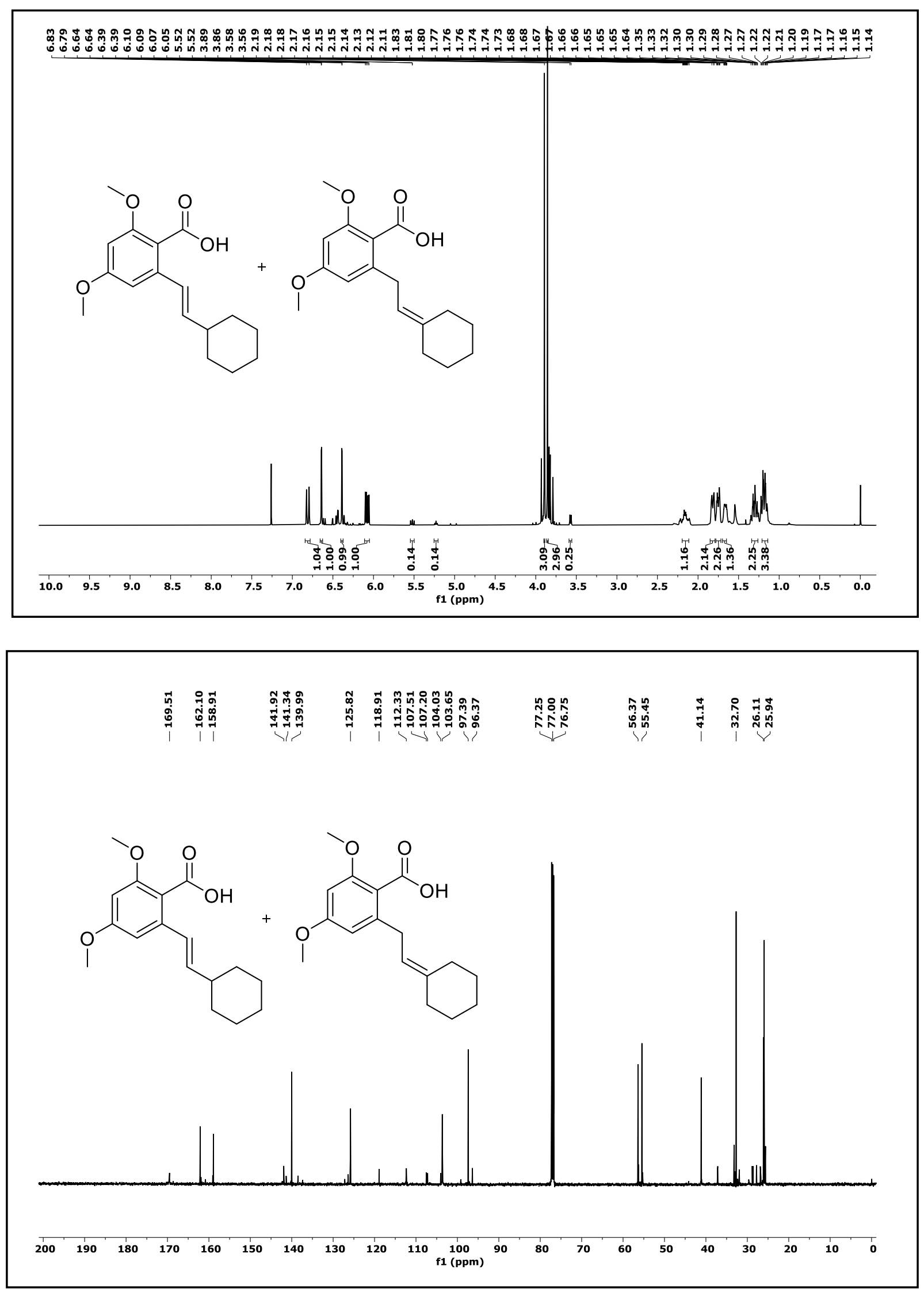
${ }^{1} \mathrm{H}$ and ${ }^{13} \mathrm{C}$ NMR Spectra of Compound 3ia. $\left(\mathrm{CDCl}_{3}\right.$ solvent was used, $\left.500 \mathrm{NMR} \mathrm{MHz}\right)$
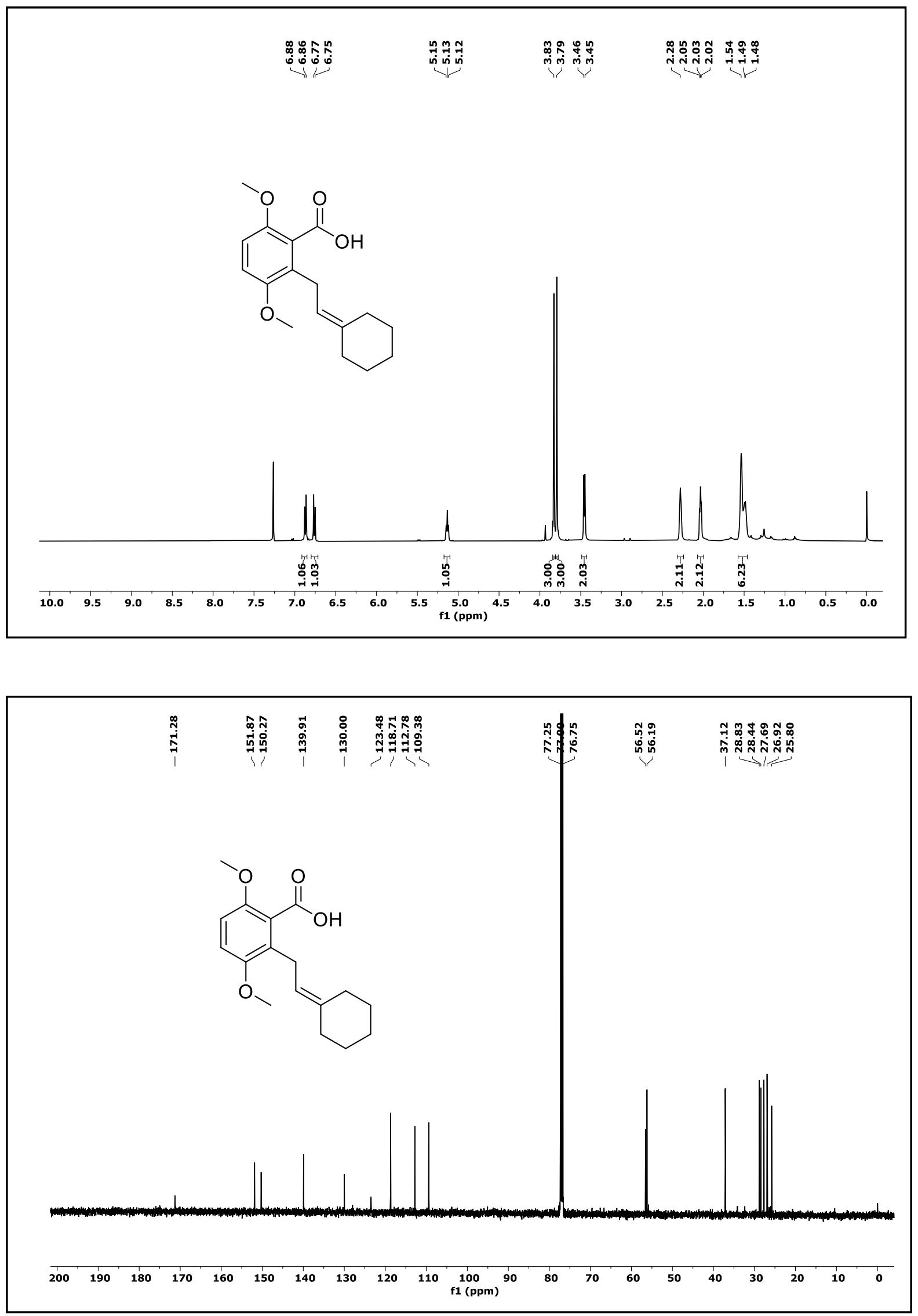
${ }^{1} \mathrm{H}$ and ${ }^{13} \mathrm{C}$ NMR Spectra of Compound 3ja. $\left(\mathrm{CDCl}_{3}\right.$ solvent was used, $\left.500 \mathrm{NMR} \mathrm{MHz}\right)$
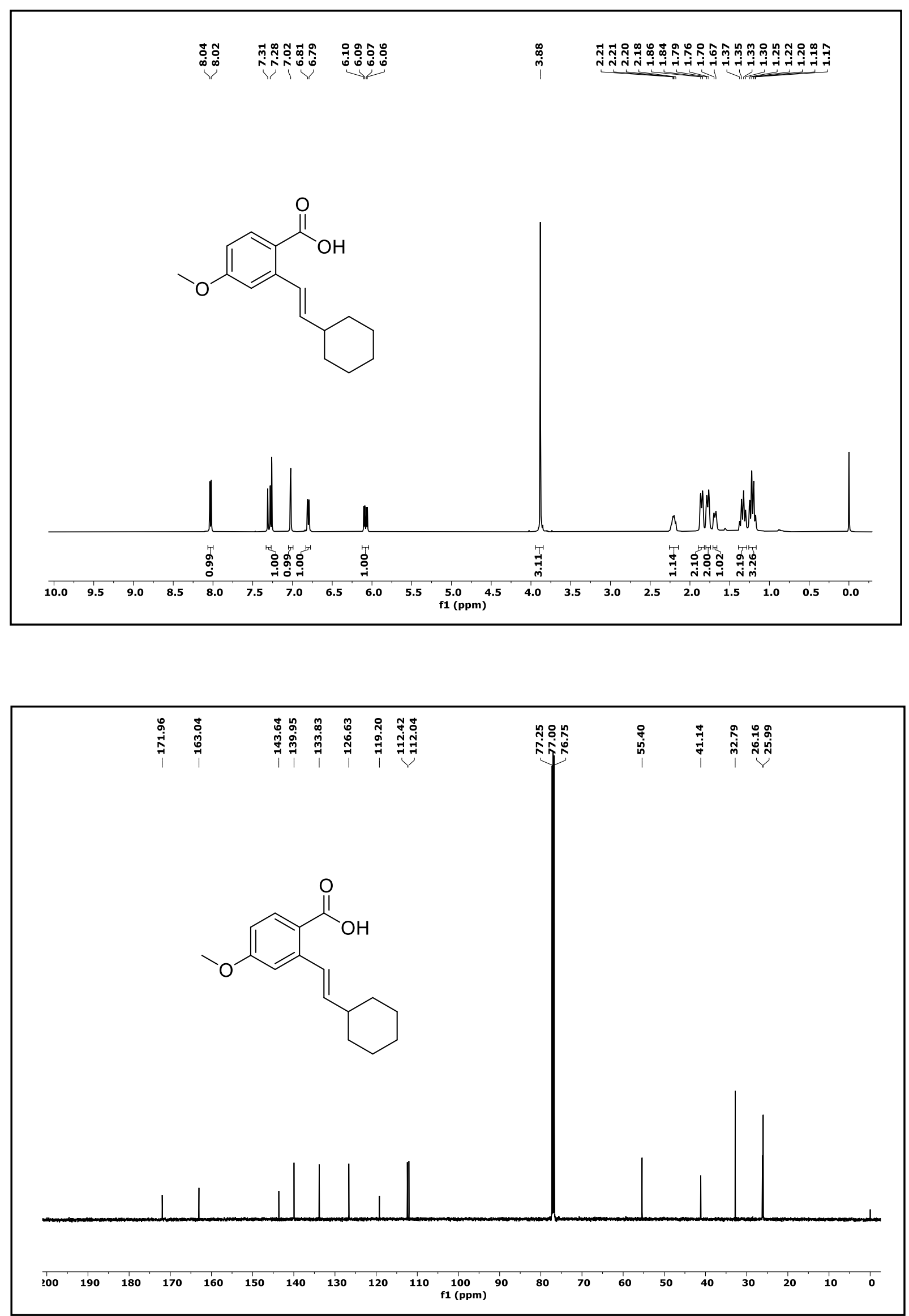
${ }^{1} \mathrm{H}$ and ${ }^{13} \mathrm{C}$ NMR Spectra of Compound 3ka. $\left(\mathrm{CDCl}_{3}\right.$ solvent was used, $\left.500 \mathrm{NMR} \mathrm{MHz}\right)$
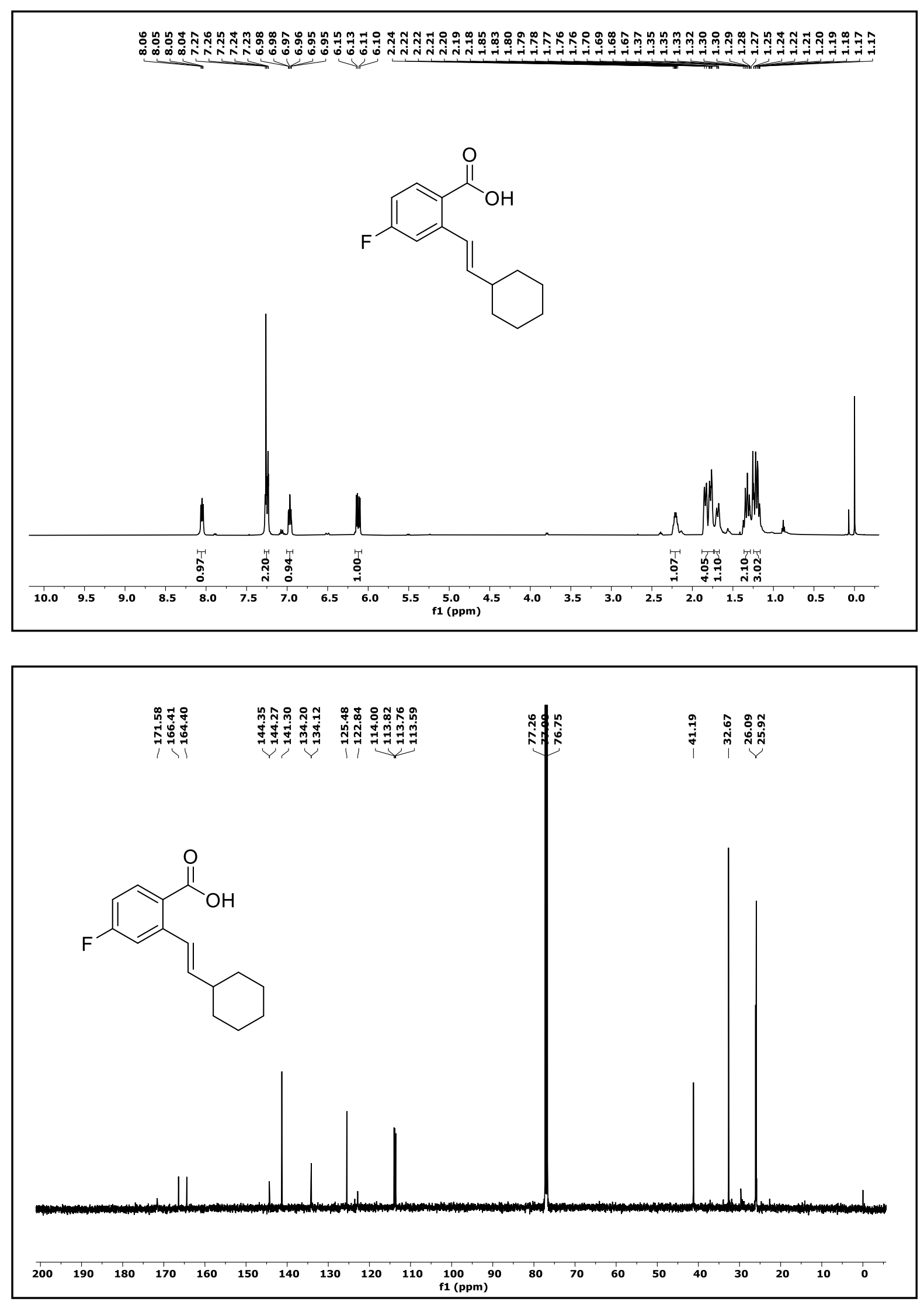
${ }^{1} \mathrm{H}$ and ${ }^{13} \mathrm{C}$ NMR Spectra of Compound 3la. $\left(\mathrm{CDCl}_{3}\right.$ solvent was used, $\left.500 \mathrm{NMR} \mathrm{MHz}\right)$
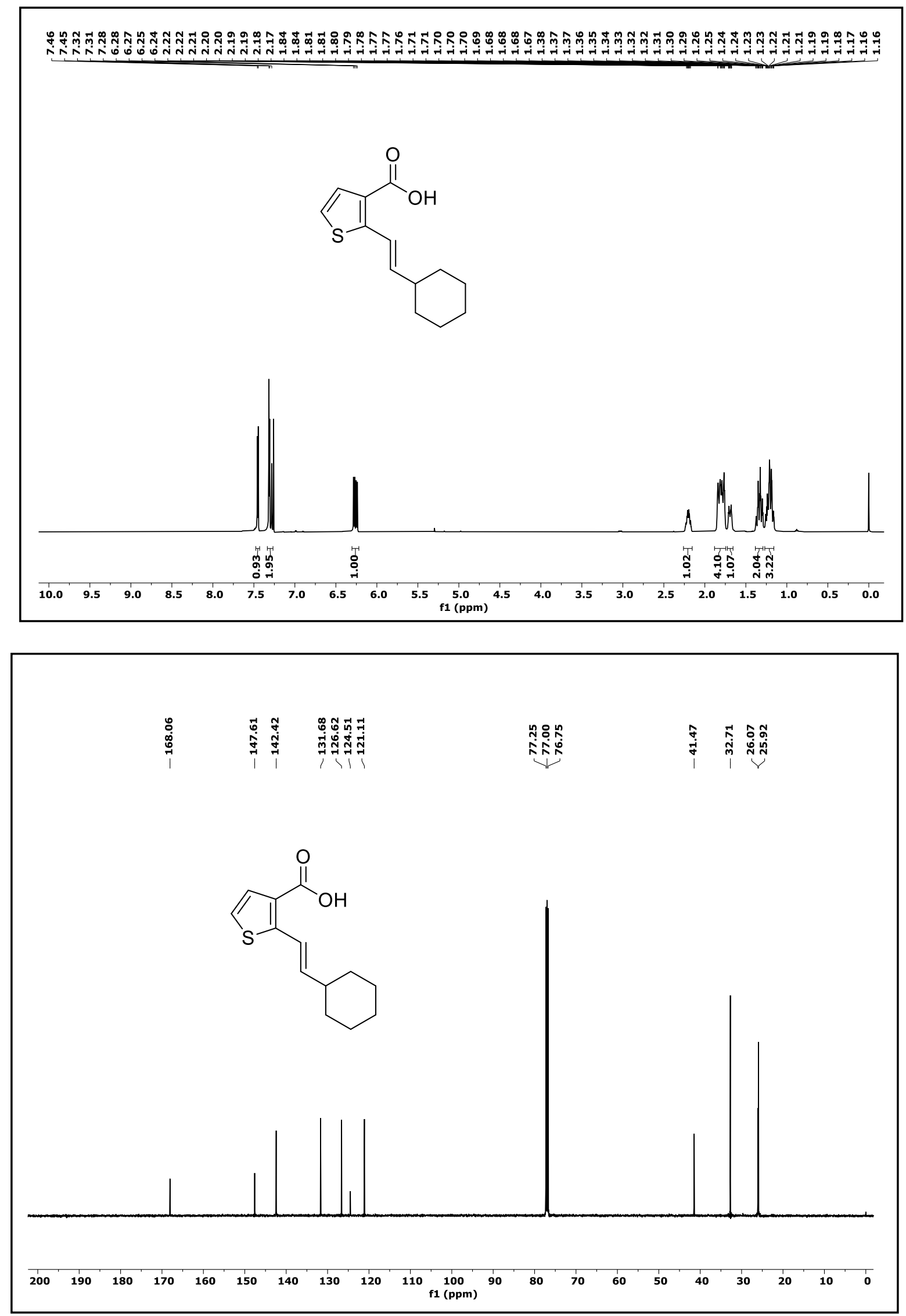
${ }^{1} \mathrm{H}$ and ${ }^{13} \mathrm{C}$ NMR Spectra of Compound 3ma. $\left(\mathrm{CDCl}_{3}\right.$ solvent was used, $\left.500 \mathrm{NMR} \mathrm{MHz}\right)$
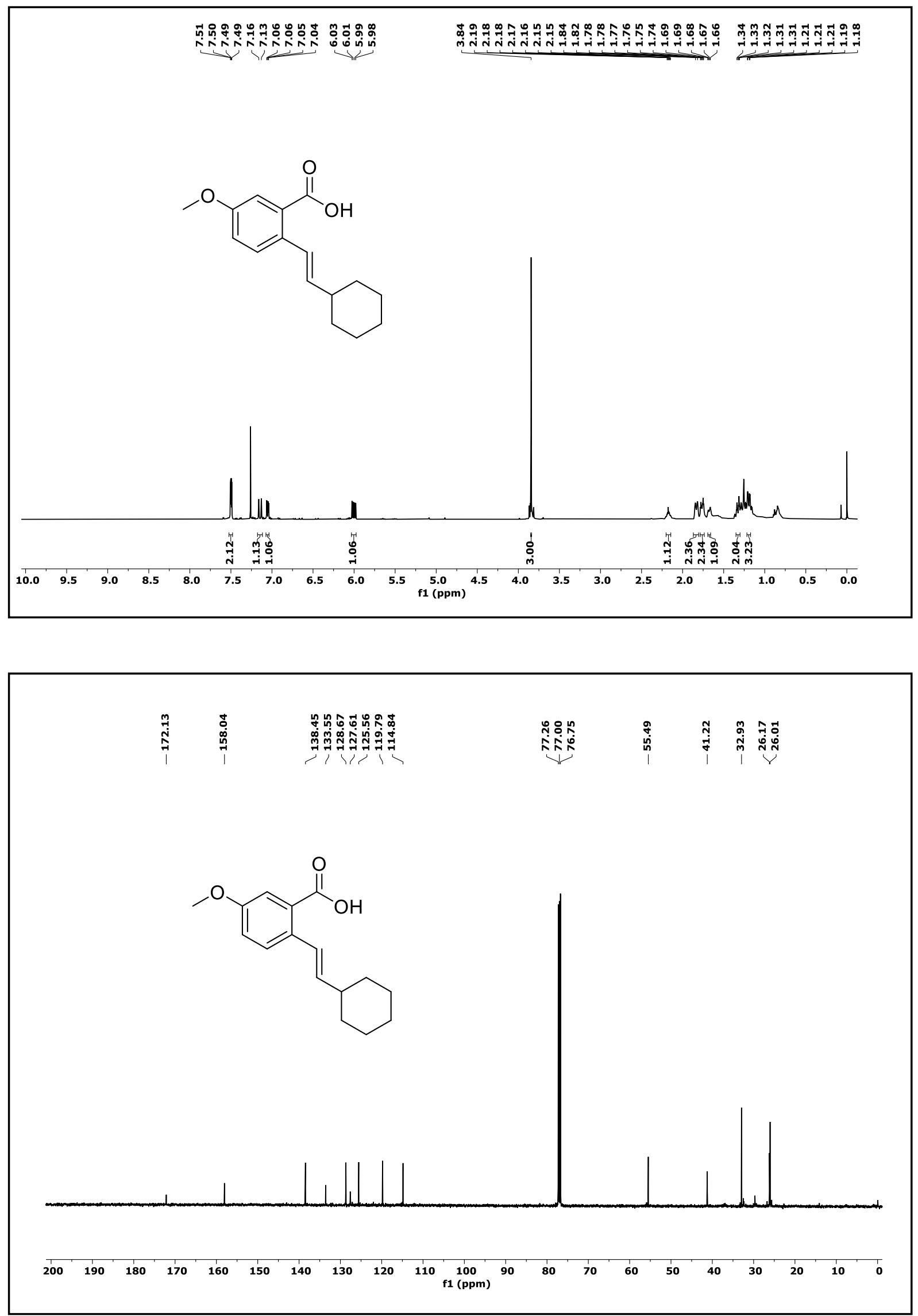
${ }^{1} \mathrm{H}$ and ${ }^{13} \mathrm{C}$ NMR Spectra of Compound 3na. $\left(\mathrm{CDCl}_{3}\right.$ solvent was used, $\left.500 \mathrm{NMR} \mathrm{MHz}\right)$
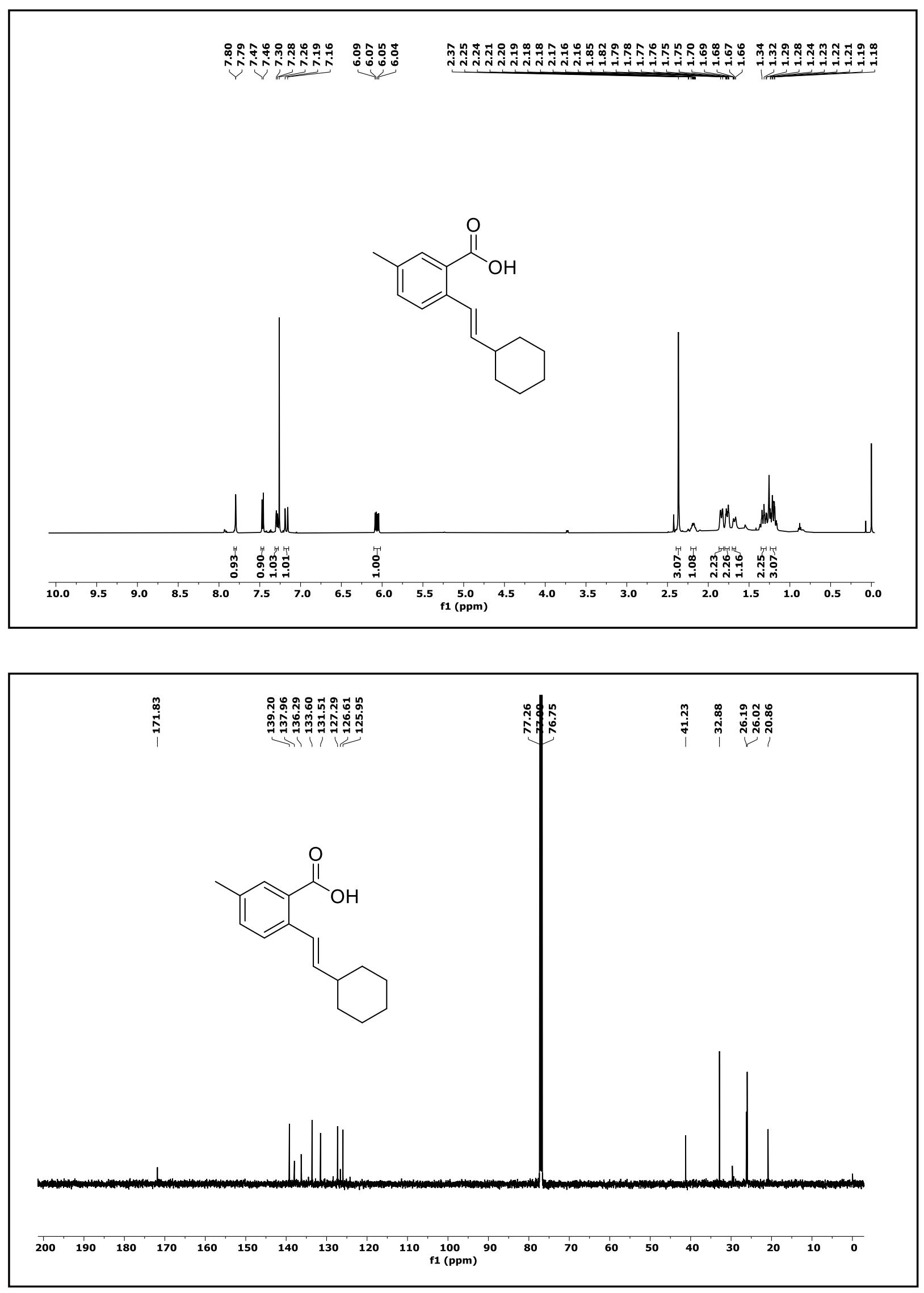
${ }^{1} \mathrm{H}$ and ${ }^{13} \mathrm{C}$ NMR Spectra of Compound 3oa. $\left(\mathrm{CDCl}_{3}\right.$ solvent was used, $\left.500 \mathrm{NMR} \mathrm{MHz}\right)$
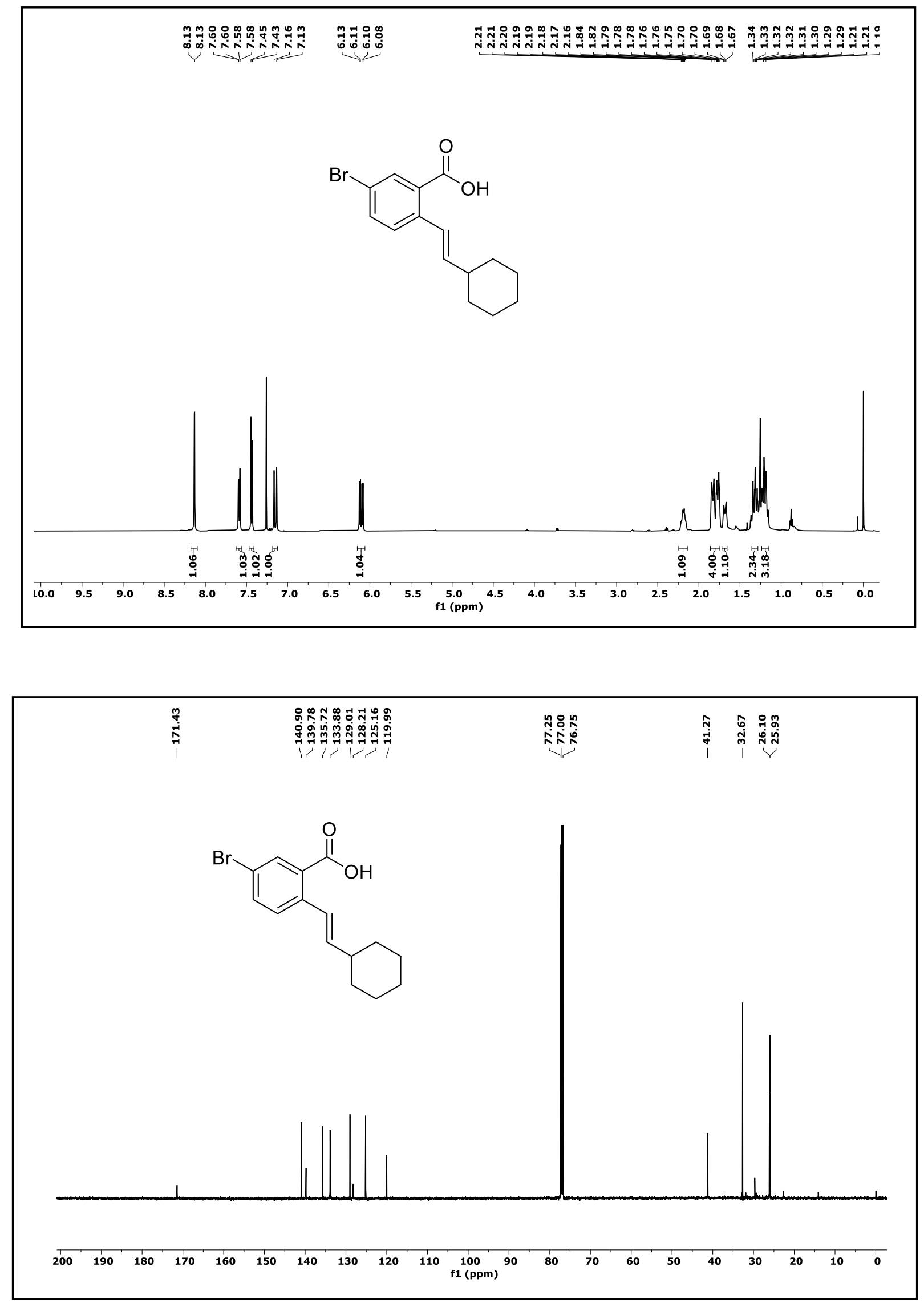
${ }^{1} \mathrm{H}$ and ${ }^{13} \mathrm{C}$ NMR Spectra of Compound 3pa. $\left(\mathrm{CDCl}_{3}\right.$ solvent was used, $\left.500 \mathrm{NMR} \mathrm{MHz}\right)$
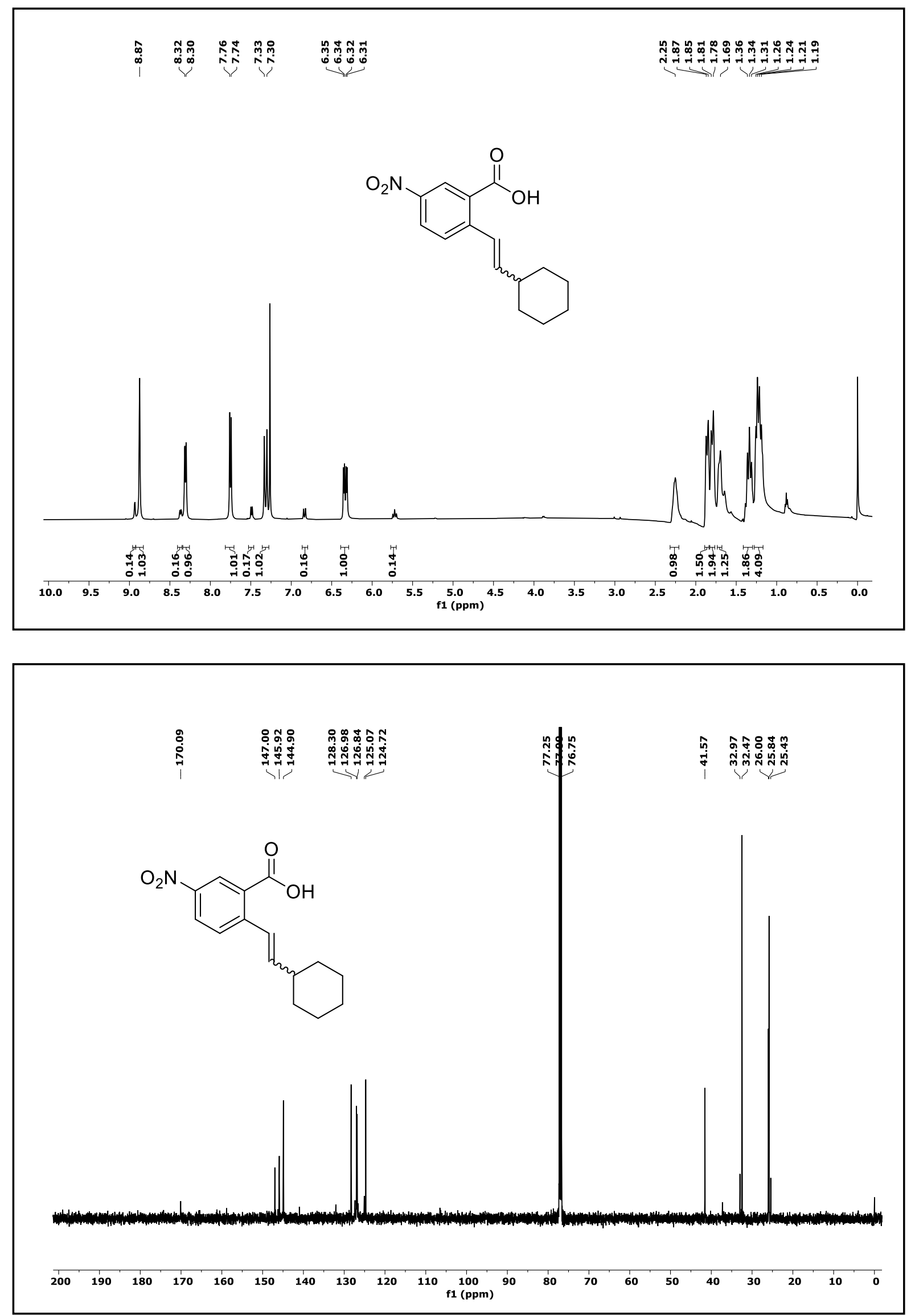
${ }^{1} \mathrm{H}$ and ${ }^{13} \mathrm{C}$ NMR Spectra of Compound 3qa. $\left(\mathrm{CDCl}_{3}\right.$ solvent was used, $\left.500 \mathrm{NMR} \mathrm{MHz}\right)$
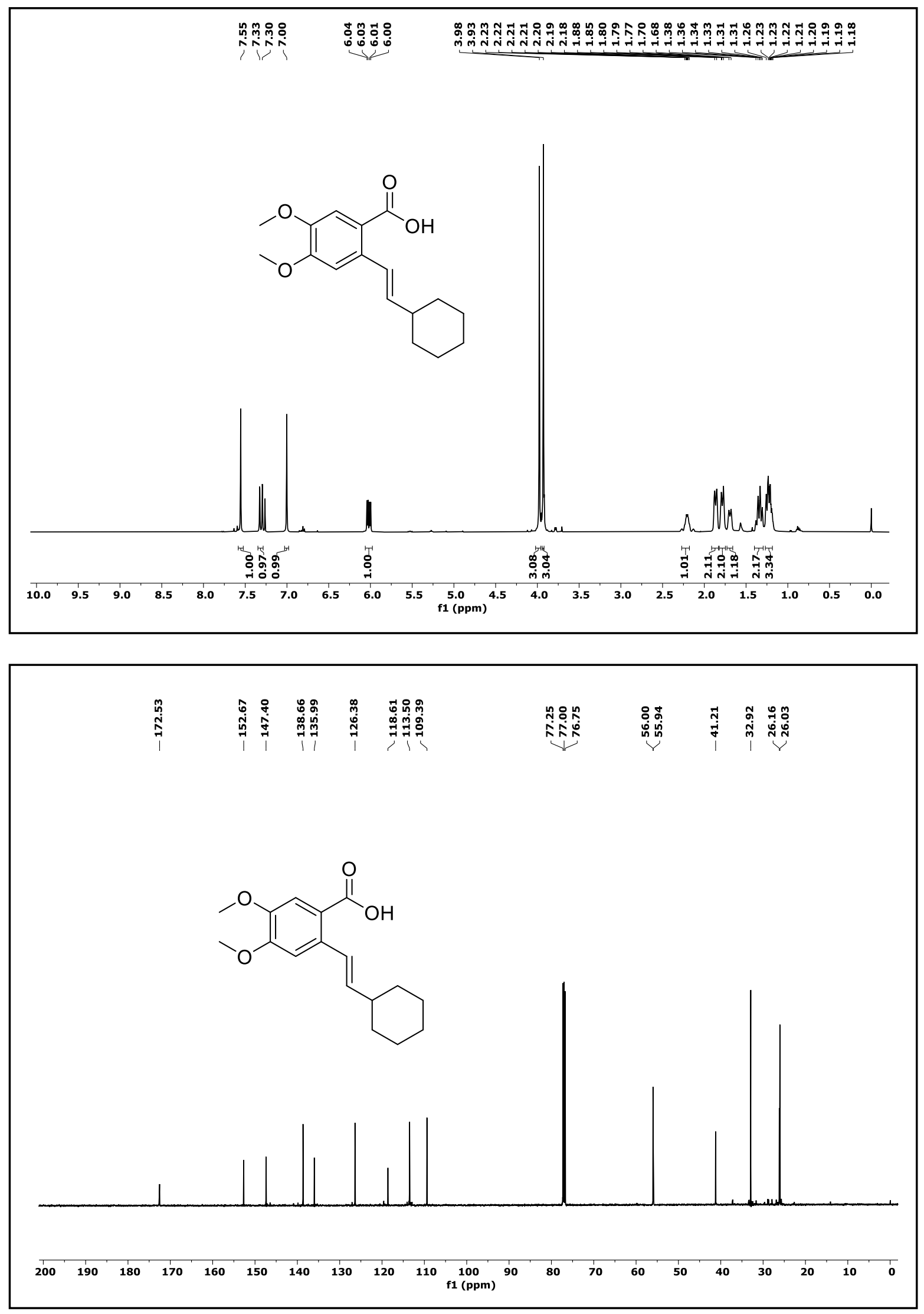
${ }^{1} \mathrm{H}$ and ${ }^{13} \mathrm{C}$ NMR Spectra of Compound 3ra. $\left(\mathrm{CDCl}_{3}\right.$ solvent was used, $\left.500 \mathrm{NMR} \mathrm{MHz}\right)$
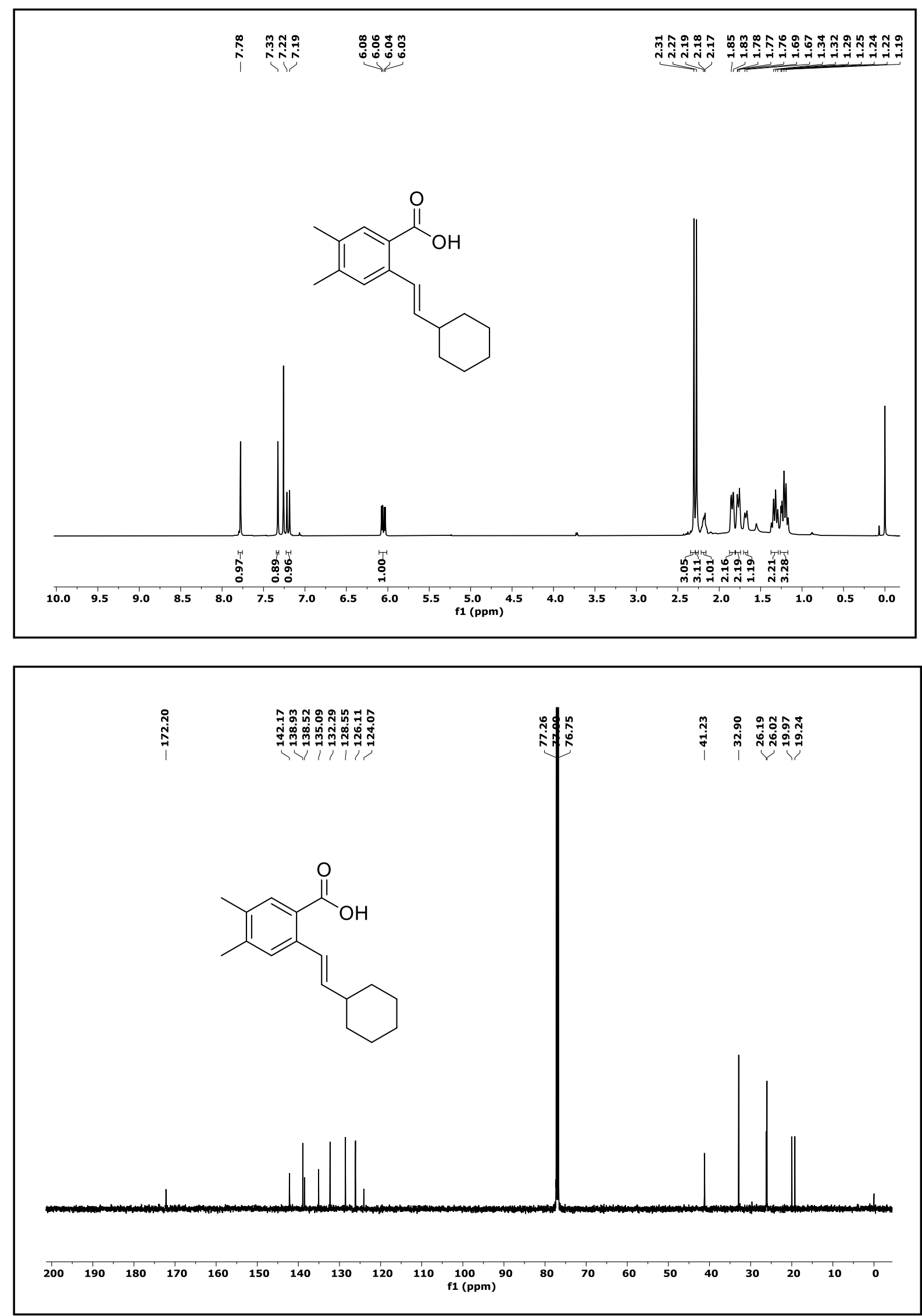
${ }^{1} \mathrm{H}$ and ${ }^{13} \mathrm{C}$ NMR Spectra of Compound 3sa. $\left(\mathrm{CDCl}_{3}\right.$ solvent was used, $\left.500 \mathrm{NMR} \mathrm{MHz}\right)$
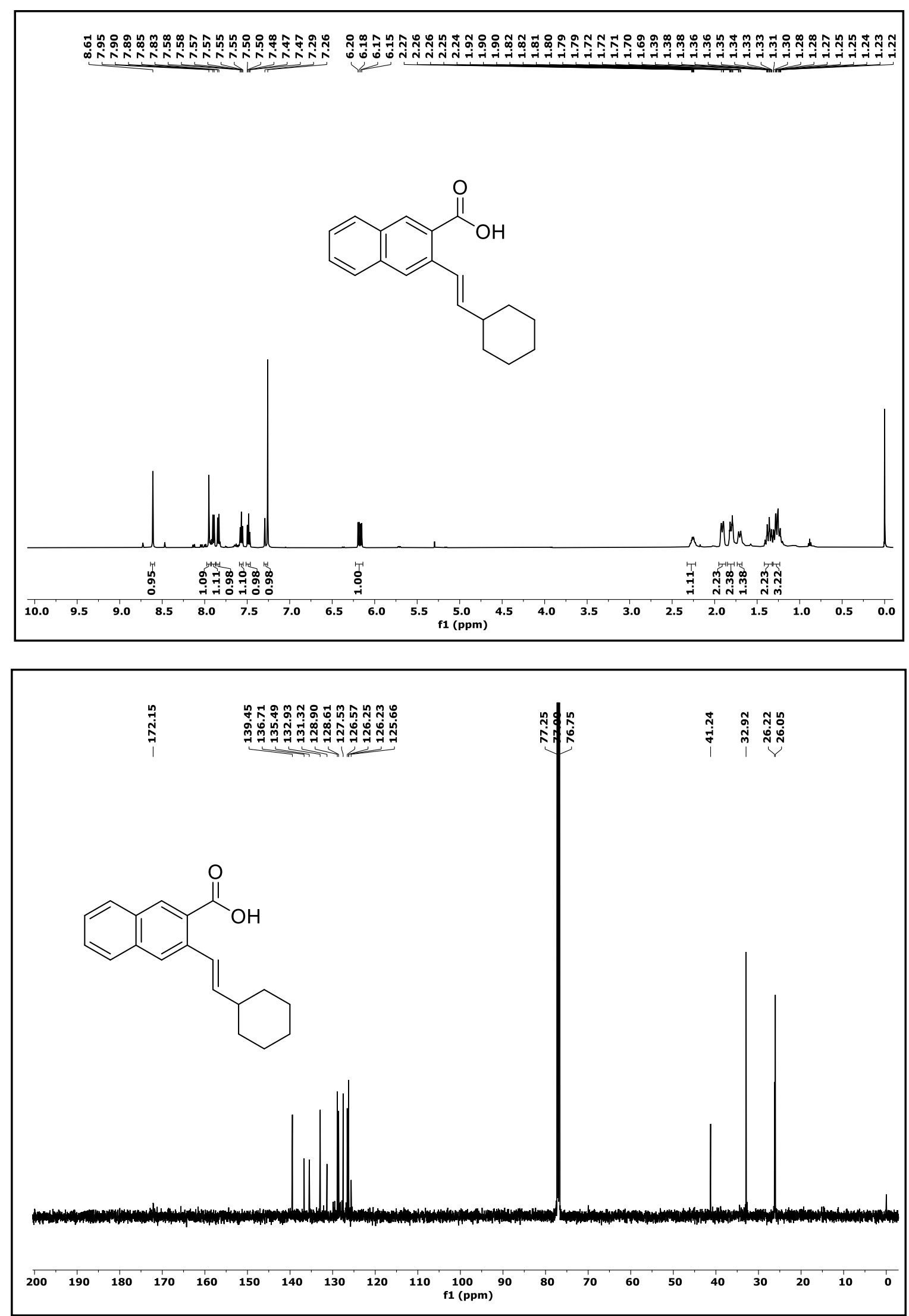
${ }^{1} \mathrm{H}$ and ${ }^{13} \mathrm{C}$ NMR Spectra of Compound $4 \mathrm{gb} .\left(\mathrm{CDCl}_{3}\right.$ solvent was used, $\left.500 \mathrm{NMR} \mathrm{MHz}\right)$
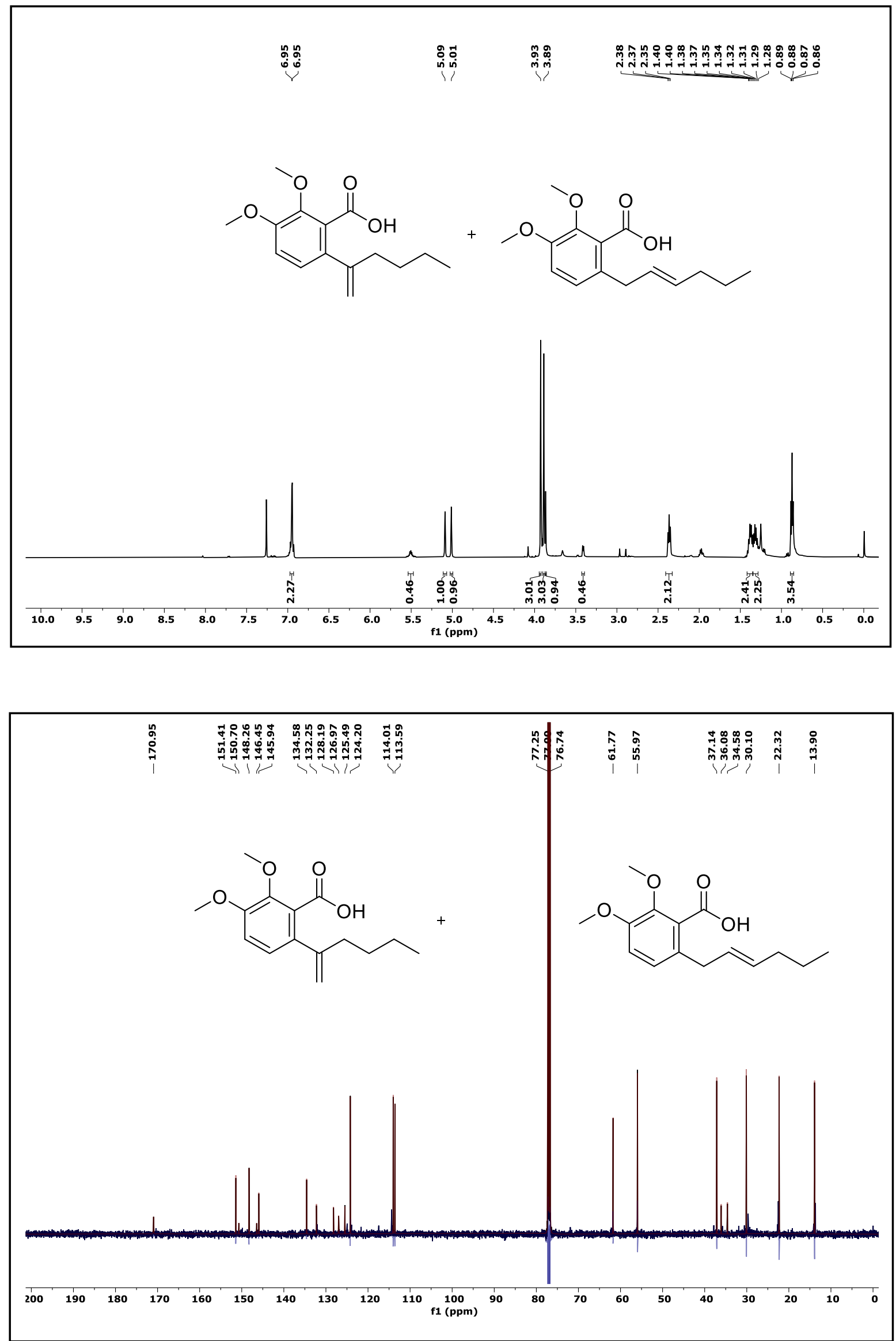
${ }^{1} \mathrm{H}$ and ${ }^{13} \mathrm{C}$ NMR Spectra of Compound 4gc. $\left(\mathrm{CDCl}_{3}\right.$ solvent was used, $\left.500 \mathrm{NMR} \mathrm{MHz}\right)$
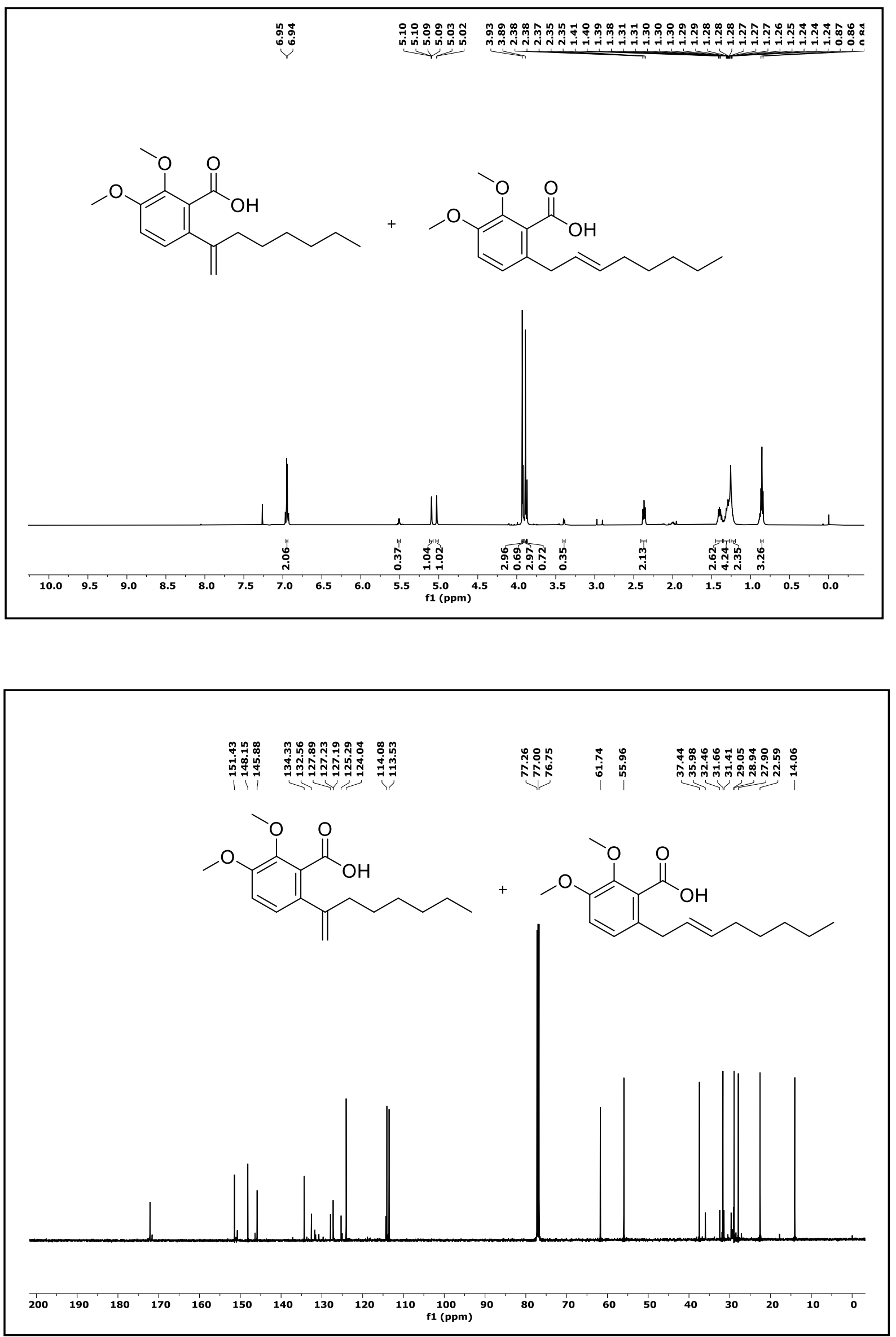
${ }^{1} \mathrm{H}$ and ${ }^{13} \mathrm{C}$ NMR Spectra of Compound 4gd. $\left(\mathrm{CDCl}_{3}\right.$ solvent was used, $\left.500 \mathrm{NMR} \mathrm{MHz}\right)$
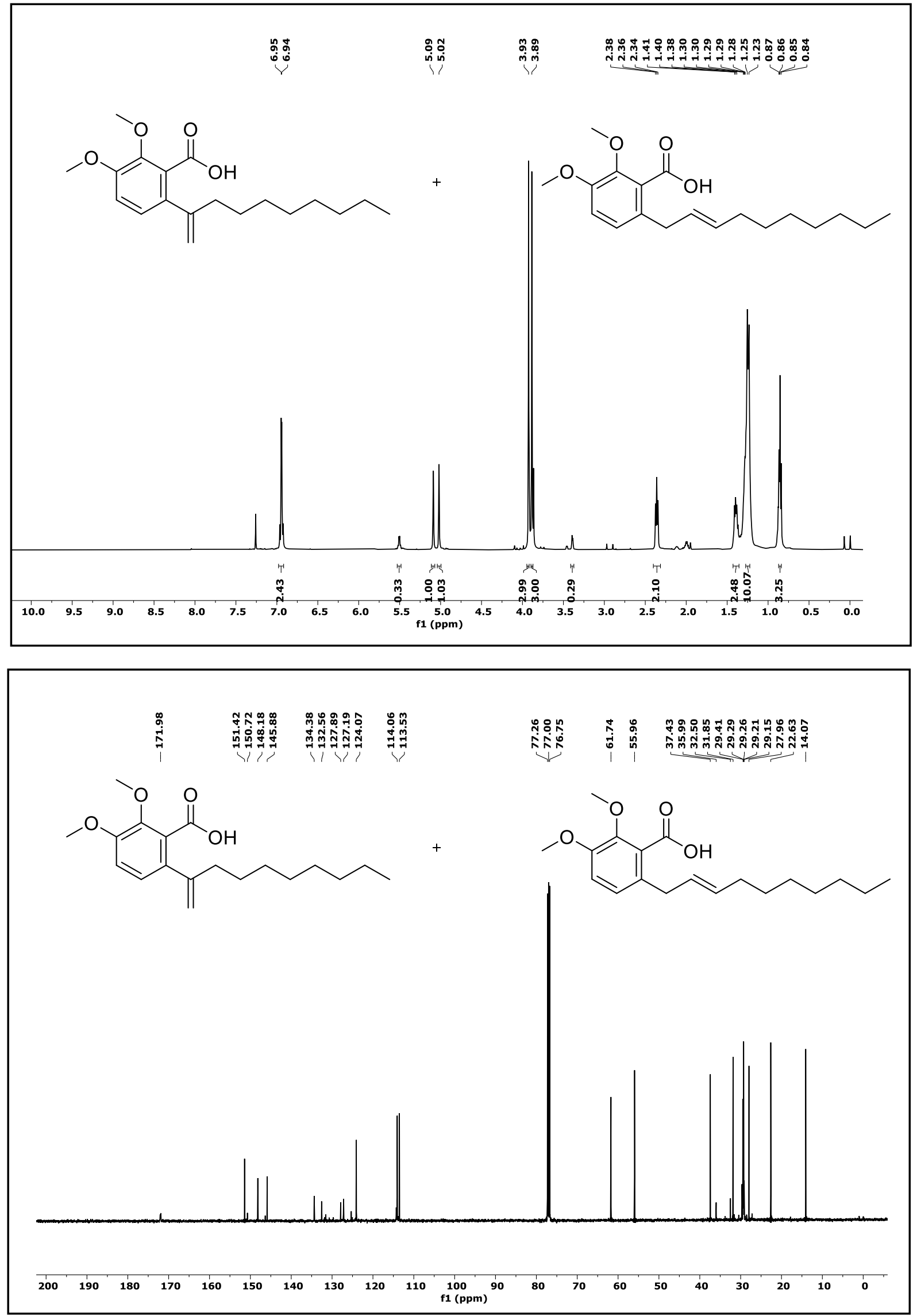
${ }^{1} \mathrm{H}$ and ${ }^{13} \mathrm{C}$ NMR Spectra of Compound 5ib. $\left(\mathrm{CDCl}_{3}\right.$ solvent was used, $\left.500 \mathrm{NMR} \mathrm{MHz}\right)$
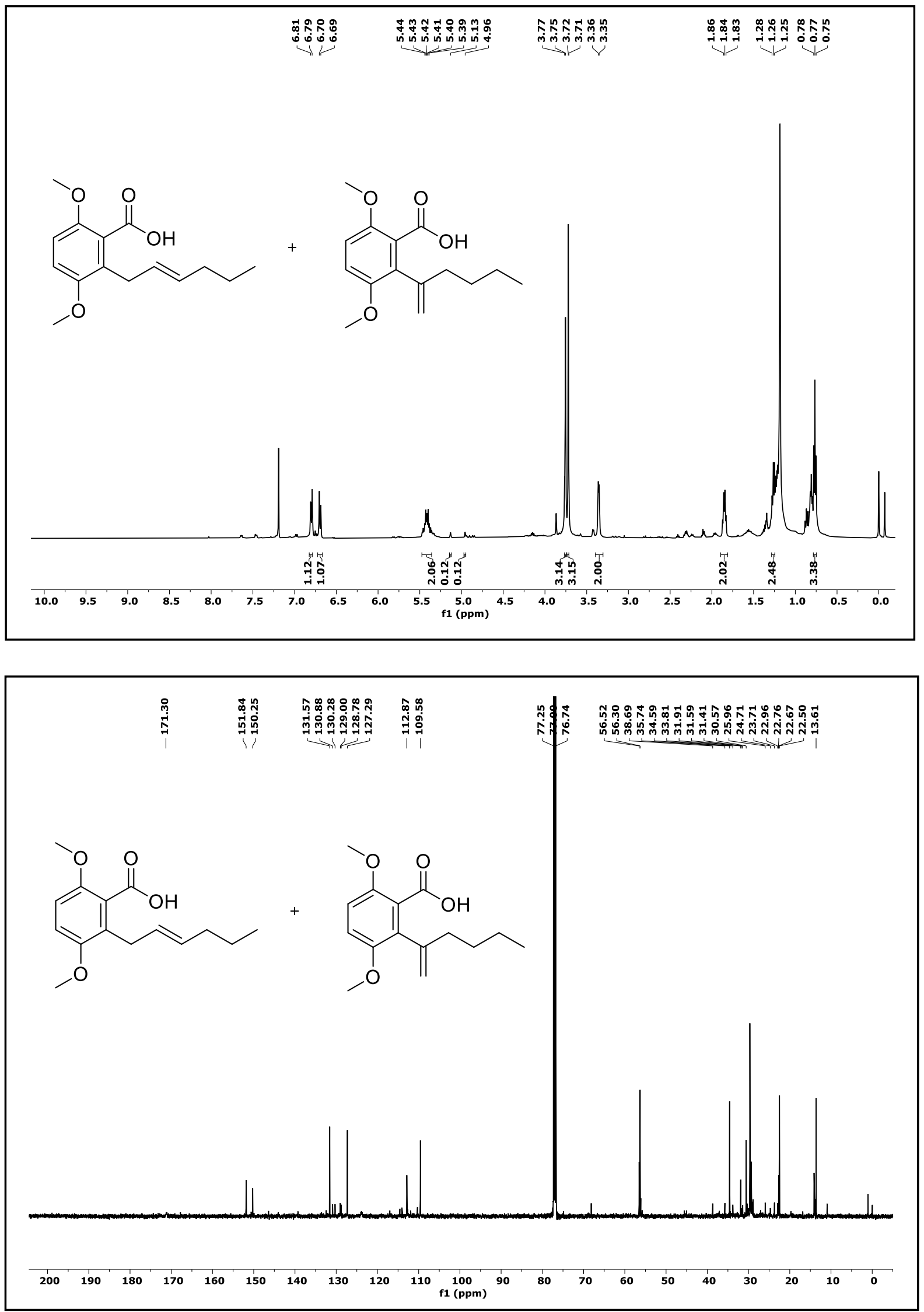
${ }^{1} \mathrm{H}$ and ${ }^{13} \mathrm{C}$ NMR Spectra of Compound 3ge. $\left(\mathrm{CDCl}_{3}\right.$ solvent was used, $\left.500 \mathrm{NMR} \mathrm{MHz}\right)$
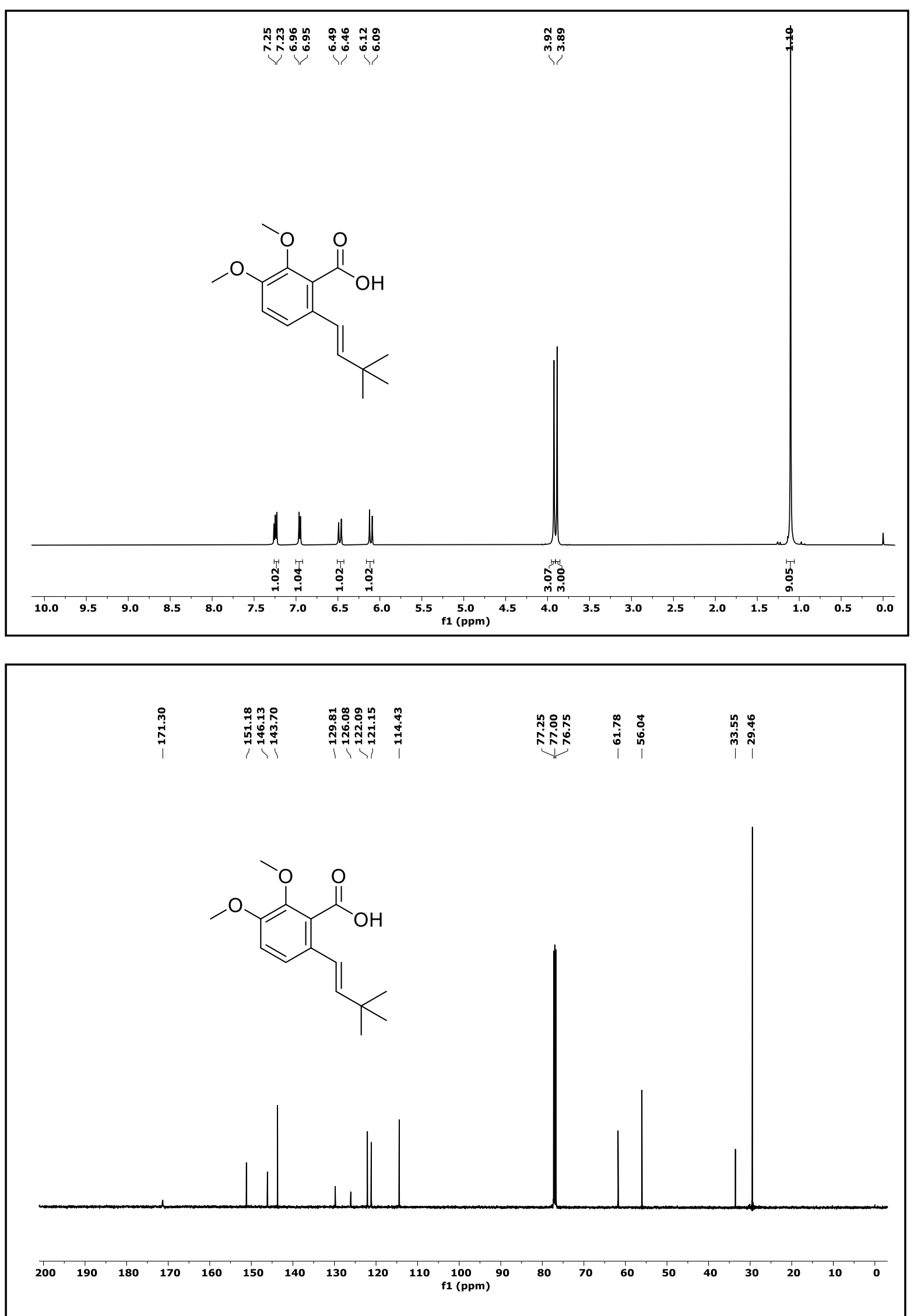
${ }^{1} \mathrm{H}$ and ${ }^{13} \mathrm{C}$ NMR Spectra of Compound 3je. $\left(\mathrm{CDCl}_{3}\right.$ solvent was used, $\left.500 \mathrm{NMR} \mathrm{MHz}\right)$
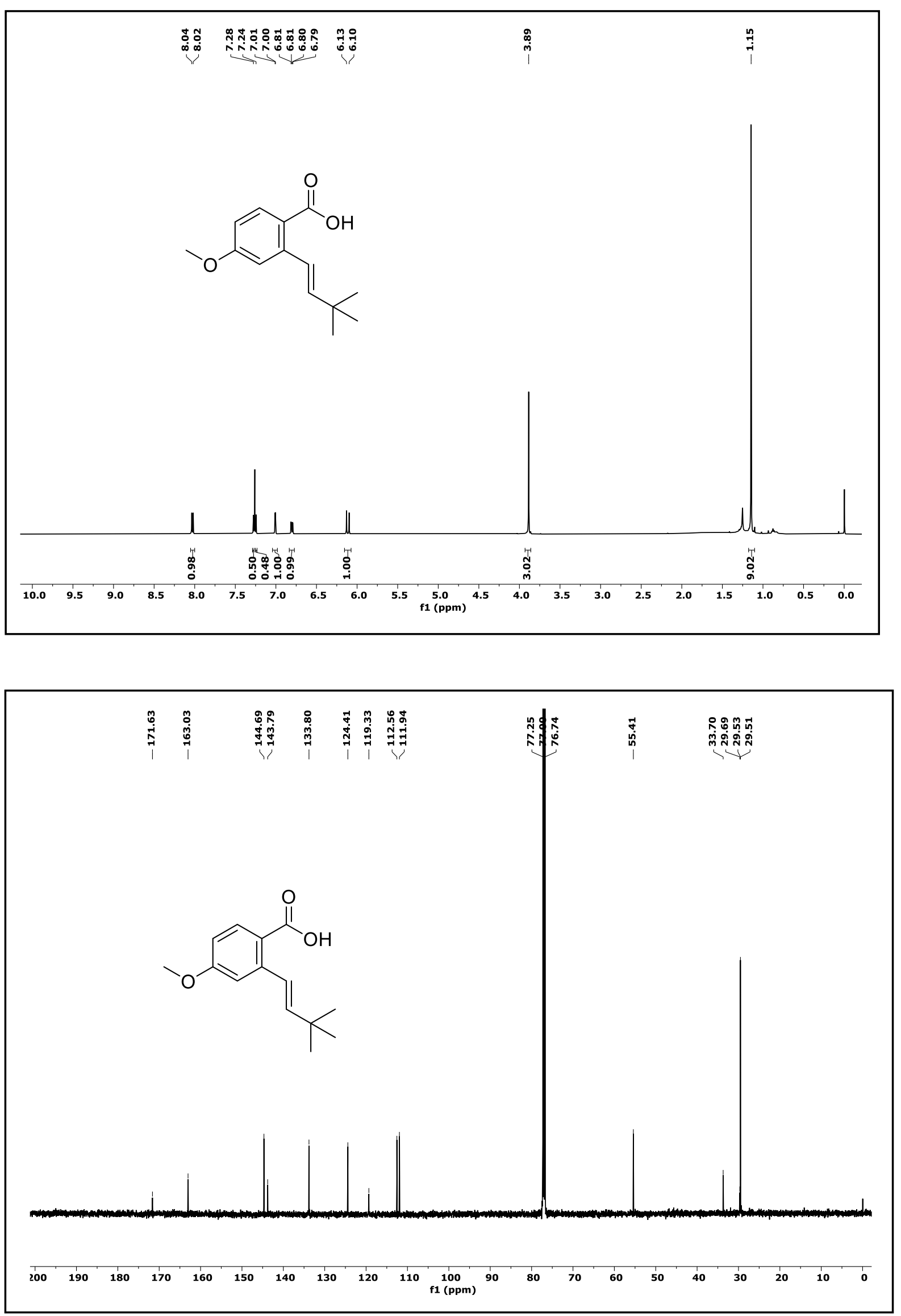
${ }^{1} \mathrm{H}$ and ${ }^{13} \mathrm{C}$ NMR Spectra of Compound 3je'. $\left(\mathrm{CDCl}_{3}\right.$ solvent was used, $\left.500 \mathrm{NMR} \mathrm{MHz}\right)$
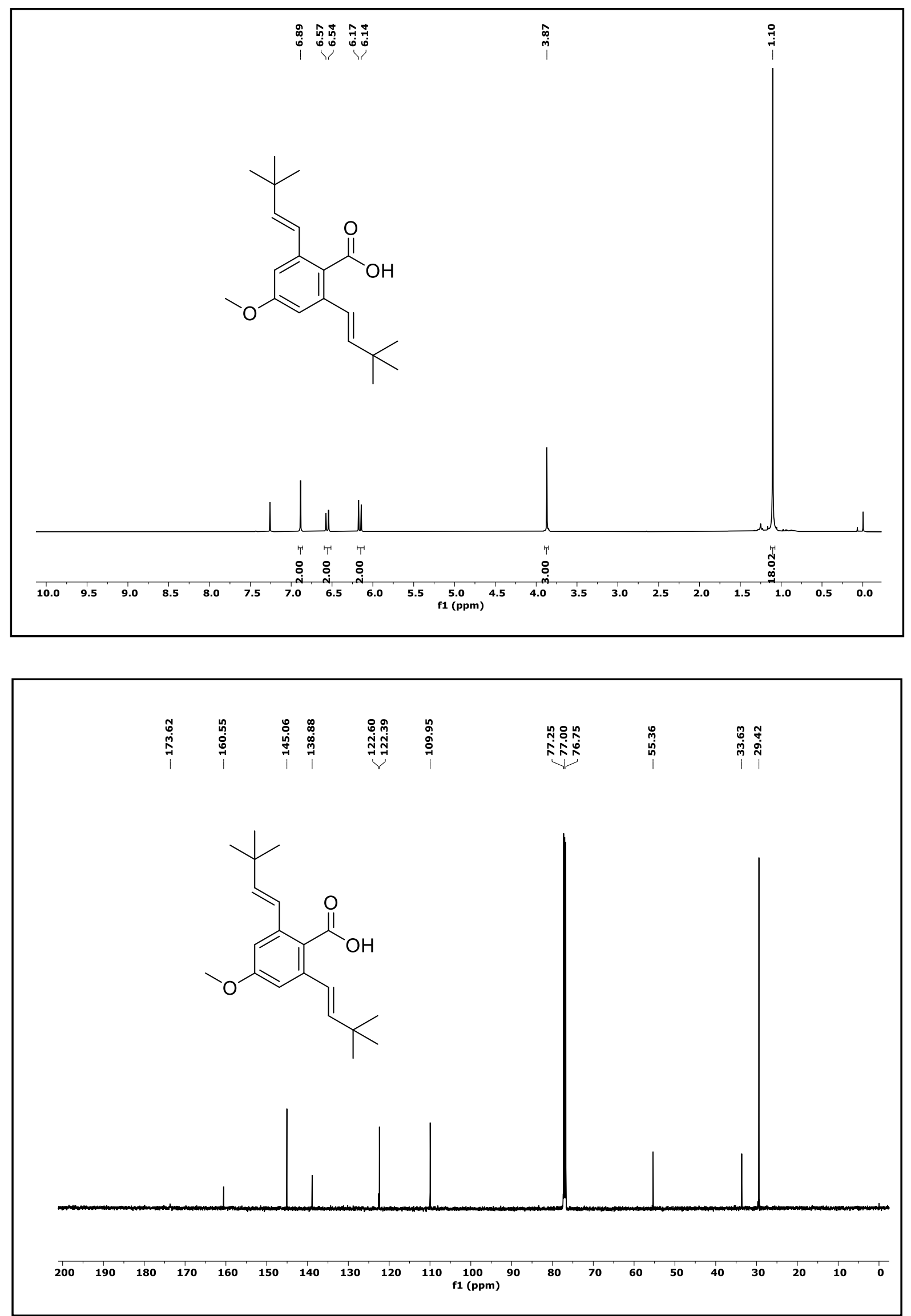
${ }^{1} \mathrm{H}$ and ${ }^{13} \mathrm{C}$ NMR Spectra of Compound 5gf. $\left(\mathrm{CDCl}_{3}\right.$ solvent was used, $\left.500 \mathrm{NMR} \mathrm{MHz}\right)$

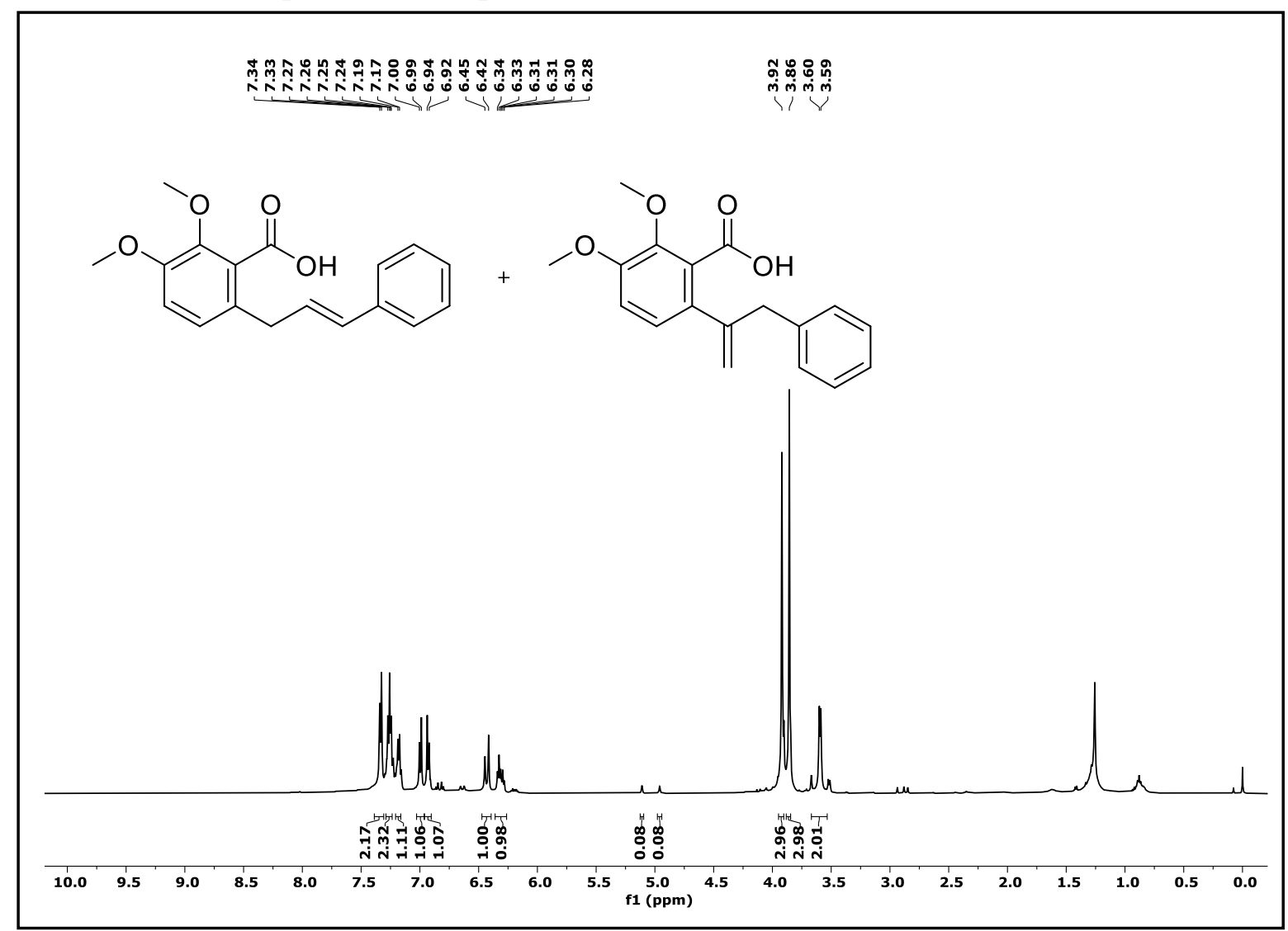

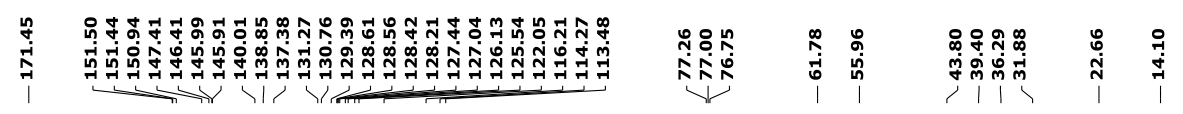

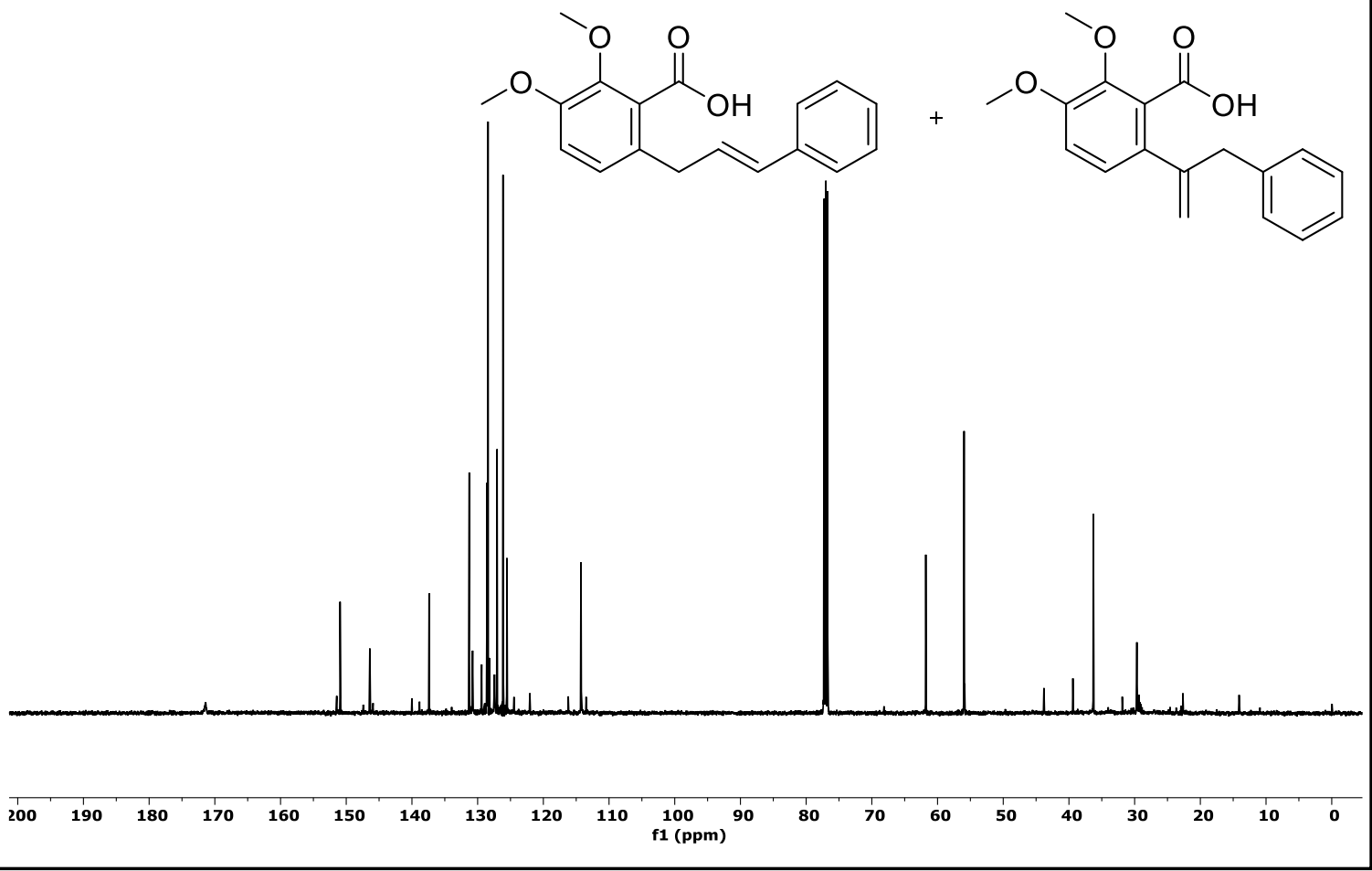


${ }^{1} \mathrm{H}$ and ${ }^{13} \mathrm{C}$ NMR Spectra of Compound 4gg. $\left(\mathrm{CDCl}_{3}\right.$ solvent was used, $\left.500 \mathrm{NMR} \mathrm{MHz}\right)$

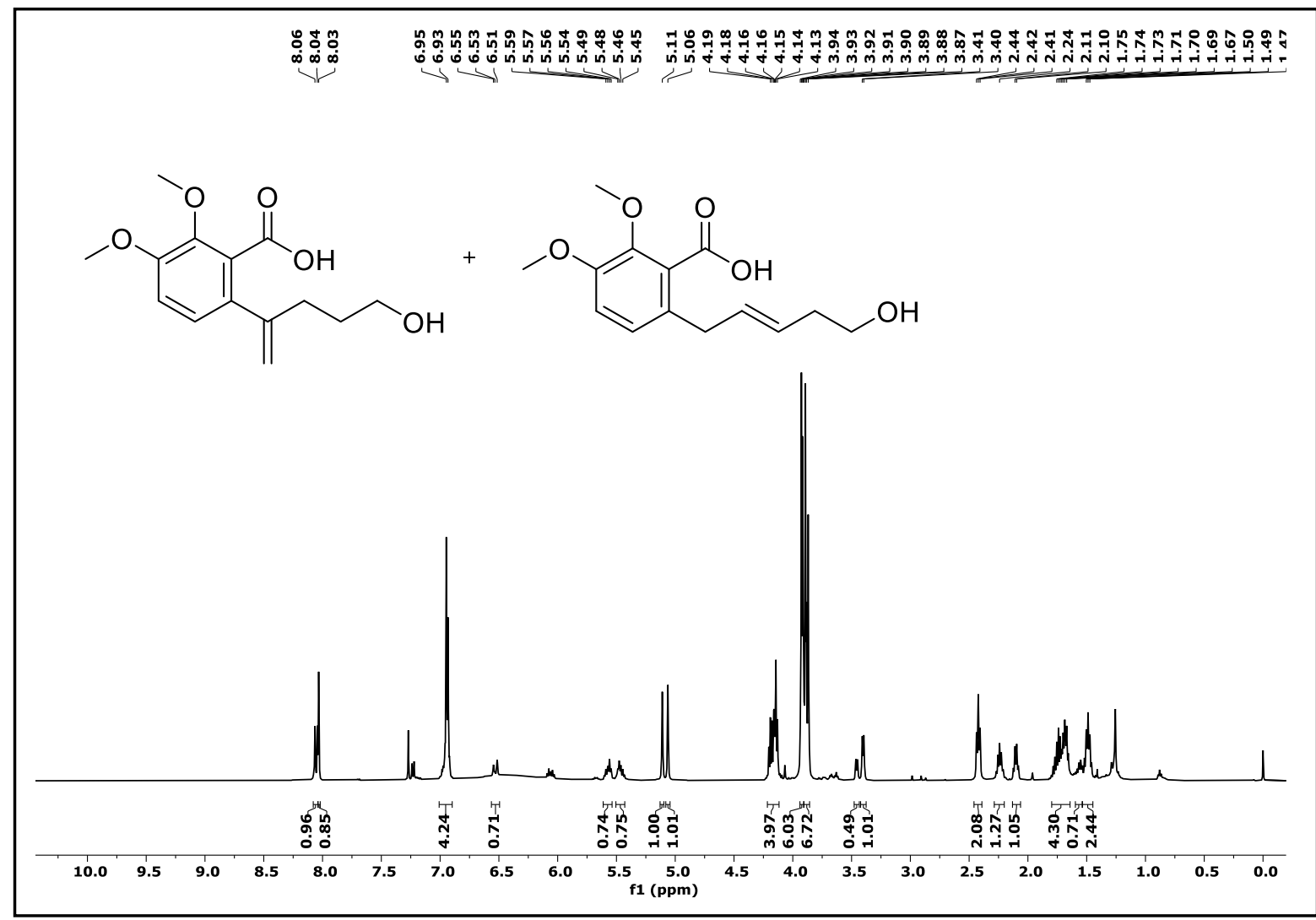

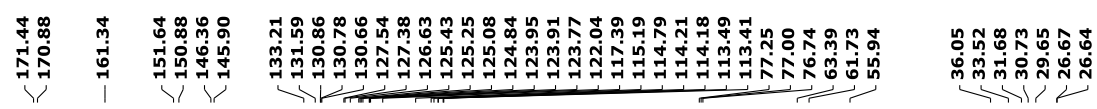
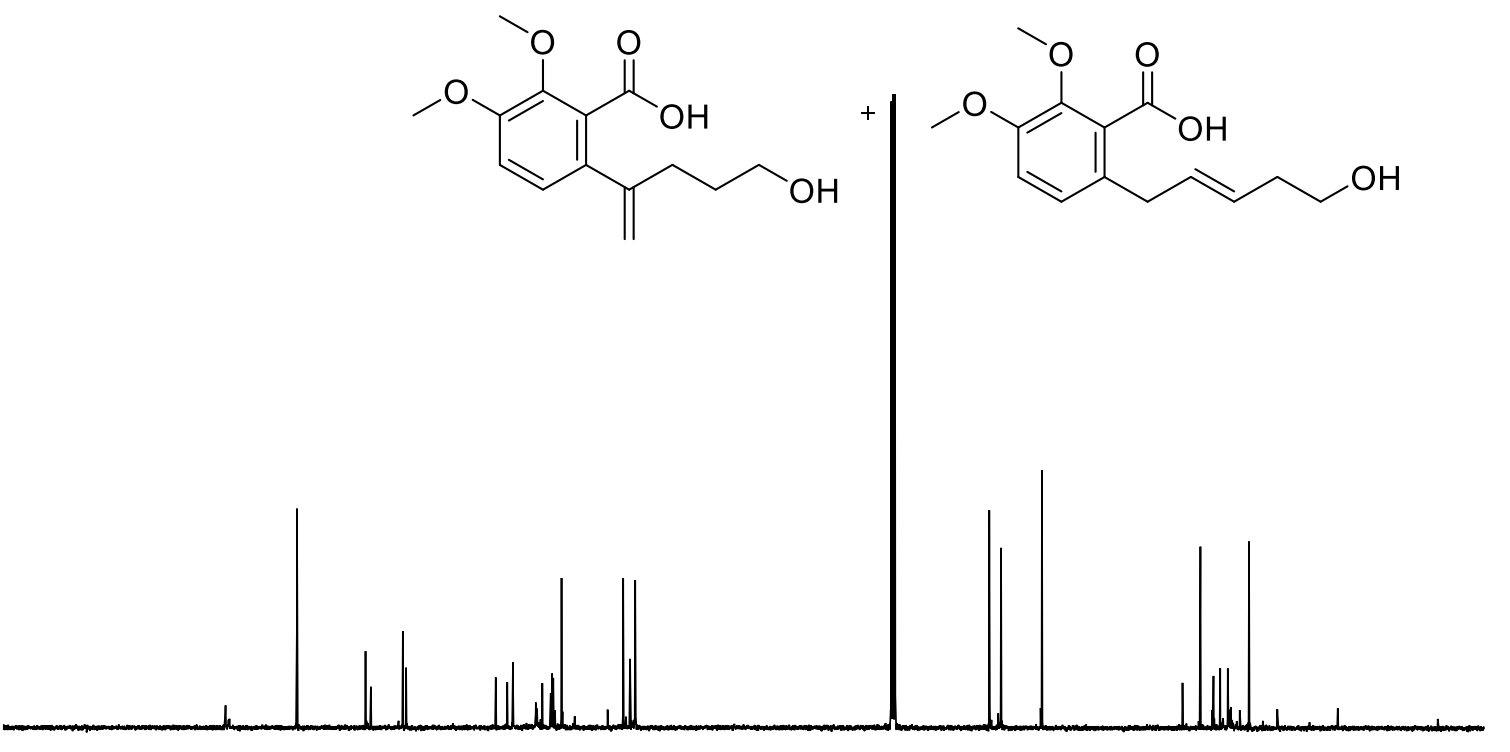

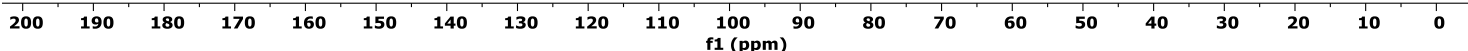


${ }^{1} \mathrm{H}$ and ${ }^{13} \mathrm{C}$ NMR Spectra of Compound 4gh. $\left(\mathrm{CDCl}_{3}\right.$ solvent was used, $\left.500 \mathrm{NMR} \mathrm{MHz}\right)$
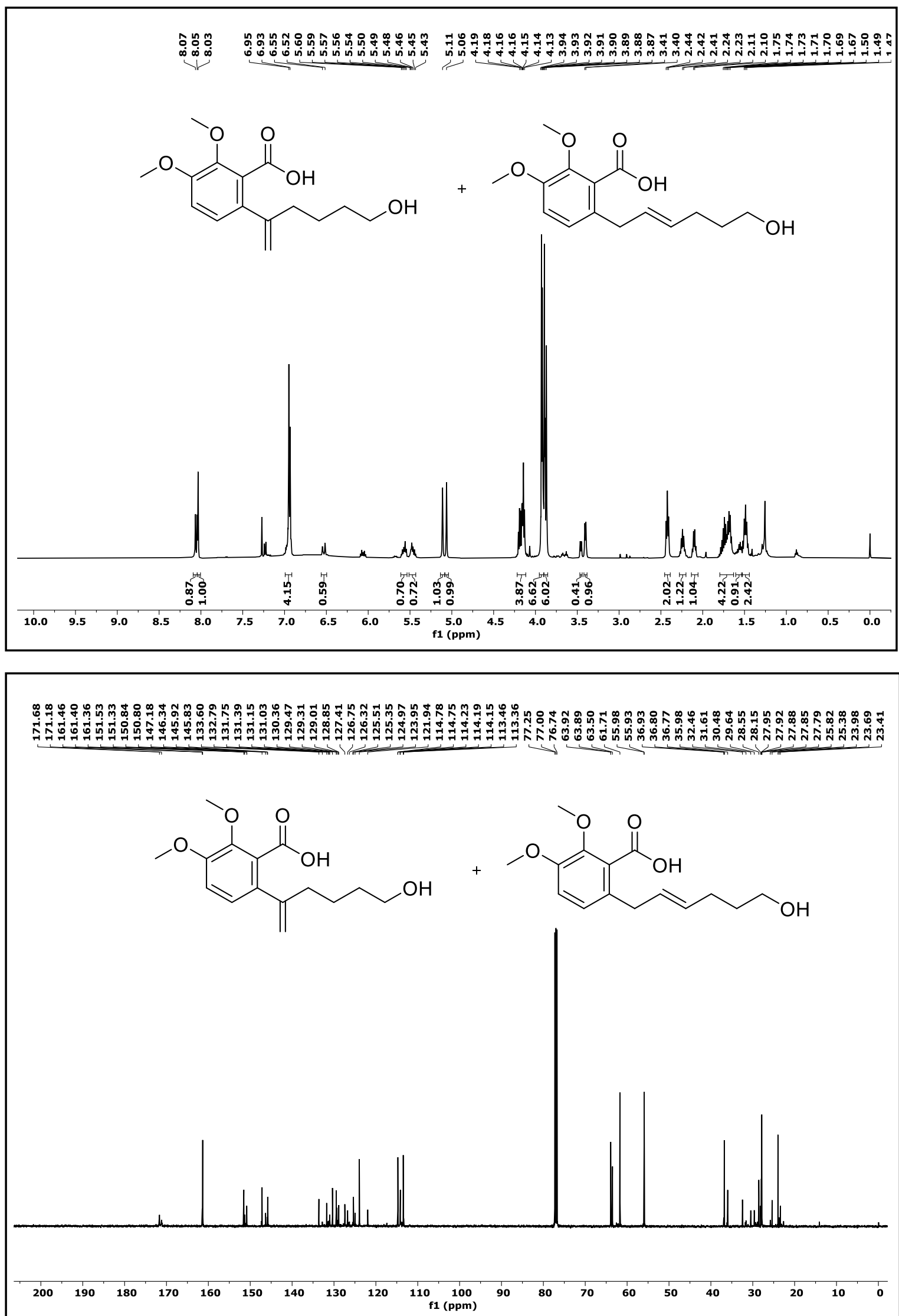
${ }^{1} \mathrm{H}$ and ${ }^{13} \mathrm{C}$ NMR Spectra of Compound 4gi. $\left(\mathrm{CDCl}_{3}\right.$ solvent was used, $\left.500 \mathrm{NMR} \mathrm{MHz}\right)$

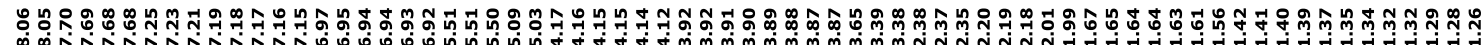<smiles>C=C(CCCCCCCCO)c1ccc(OC)c(OC)c1C(=O)O</smiles><smiles>COc1ccc(C/C=C/CCCCCCCO)c(C(=O)O)c1OC</smiles>

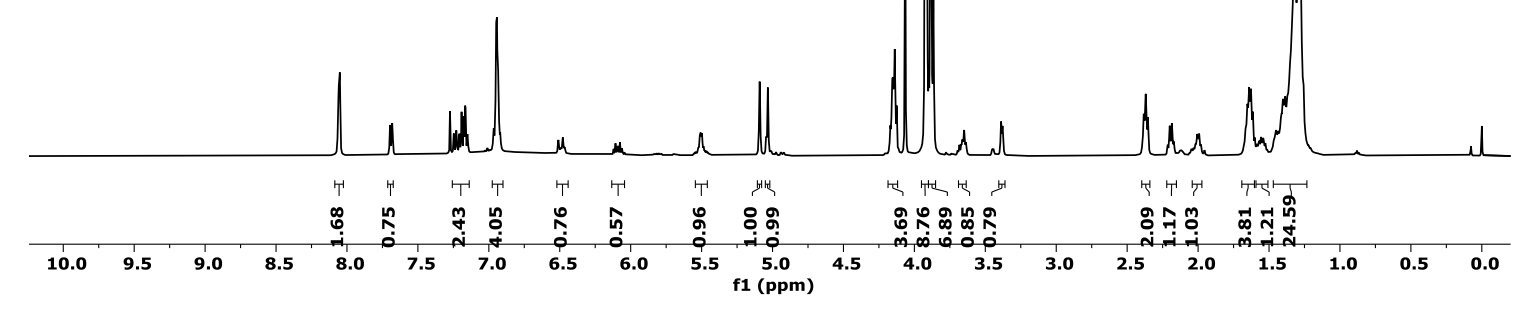

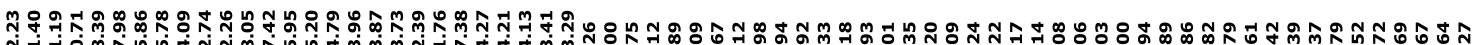

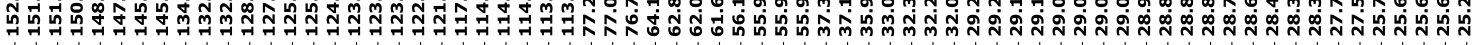<smiles>C=C(CCCCCCCCO)c1ccc(OC)c(OC)c1C(=O)O</smiles><smiles>COc1ccc(C/C=C/CCCCCCCO)c(C(=O)O)c1OC</smiles>

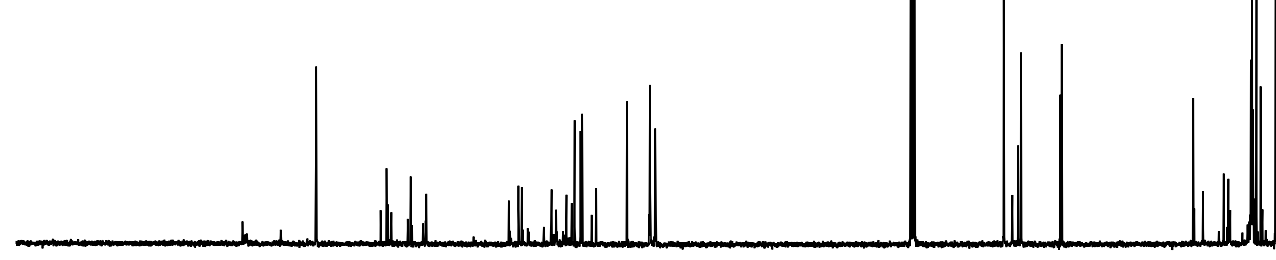

$\begin{array}{llllllllllllllllllllll}1 & 190 & 180 & 170 & 160 & 150 & 140 & 130 & 120 & 110 & \begin{array}{c}100 \\ \mathrm{f1}(\mathrm{ppm})\end{array} & 90 & 80 & 70 & 60 & 50 & 40 & 30 & 20 & 10 & 0\end{array}$ 
${ }^{1} \mathrm{H}$ and ${ }^{13} \mathrm{C}$ NMR Spectra of Compound 4ji. $\left(\mathrm{CDCl}_{3}\right.$ solvent was used, $\left.500 \mathrm{NMR} \mathrm{MHz}\right)$
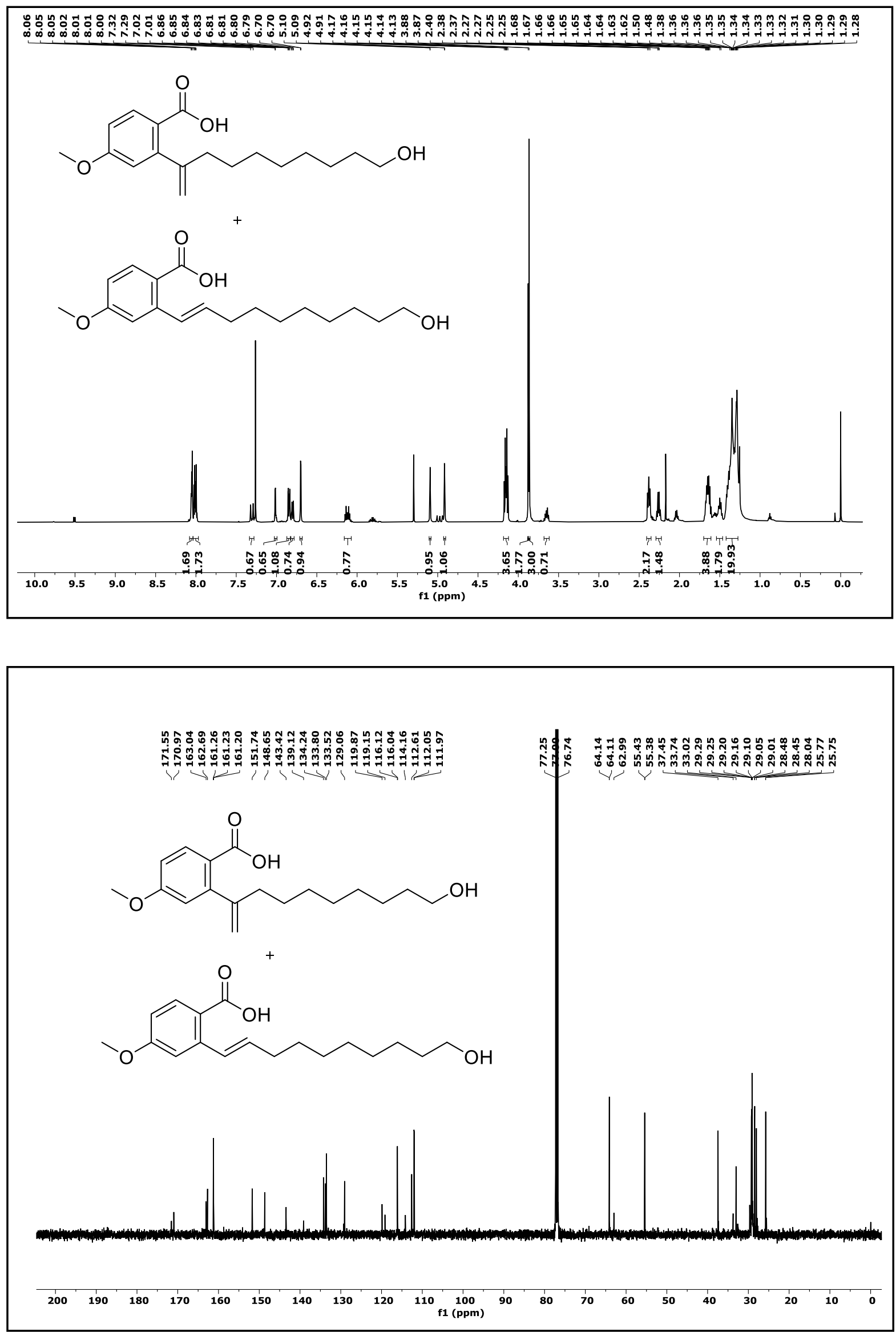
${ }^{1} \mathrm{H}$ and ${ }^{13} \mathrm{C}$ NMR Spectra of Compound 3aj. $\left(\mathrm{CDCl}_{3}\right.$ solvent was used, $\left.500 \mathrm{NMR} \mathrm{MHz}\right)$
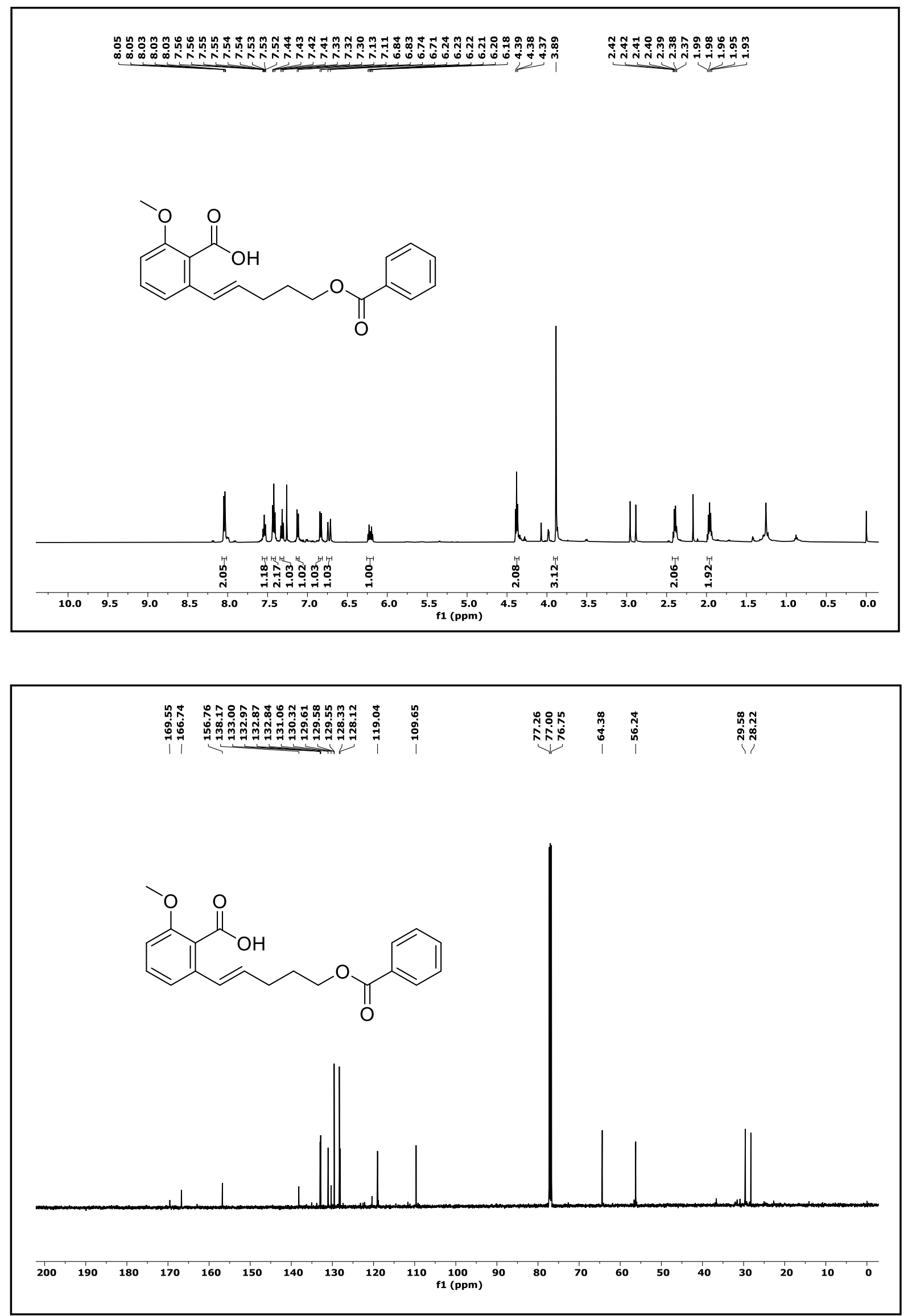
${ }^{1} \mathrm{H}$ and ${ }^{13} \mathrm{C}$ NMR Spectra of Compound 3ak. $\left(\mathrm{CDCl}_{3}\right.$ solvent was used, $\left.500 \mathrm{NMR} \mathrm{MHz}\right)$
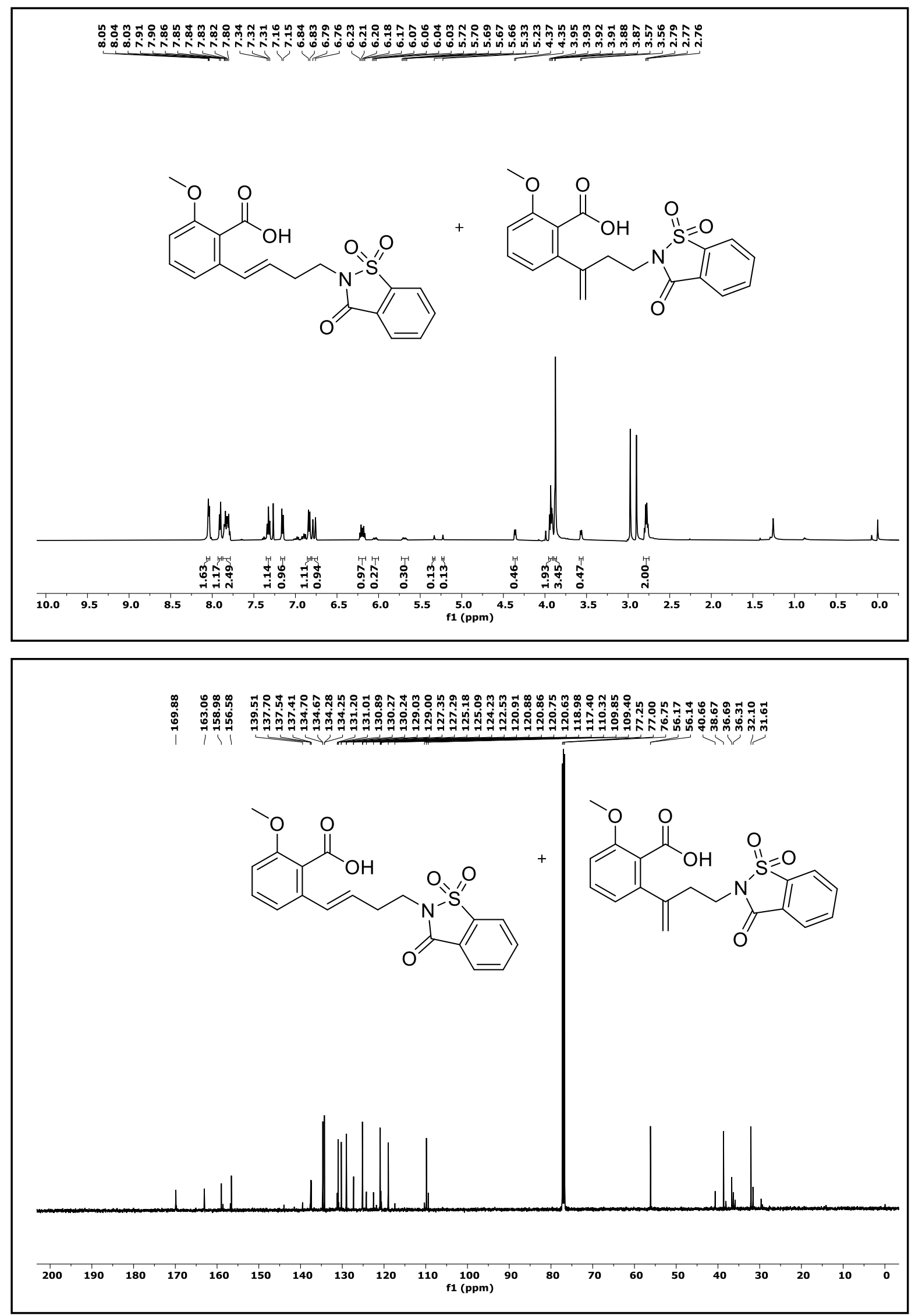
${ }^{1} \mathrm{H}$ and ${ }^{13} \mathrm{C}$ NMR Spectra of Compound 3gk. $\left(\mathrm{CDCl}_{3}\right.$ solvent was used, $\left.500 \mathrm{NMR} \mathrm{MHz}\right)$

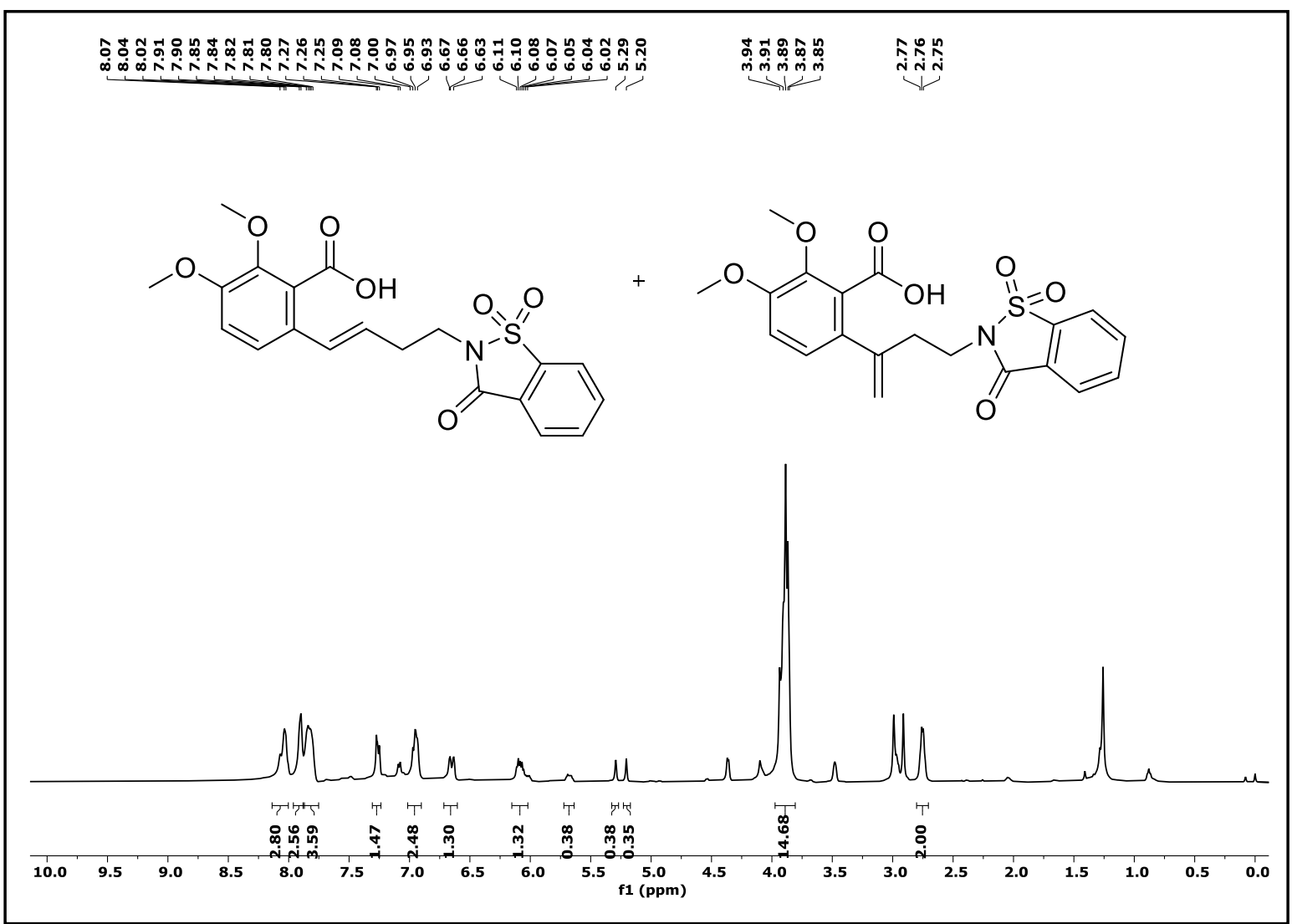

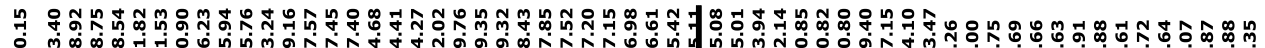

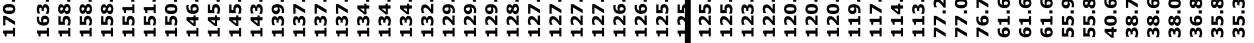

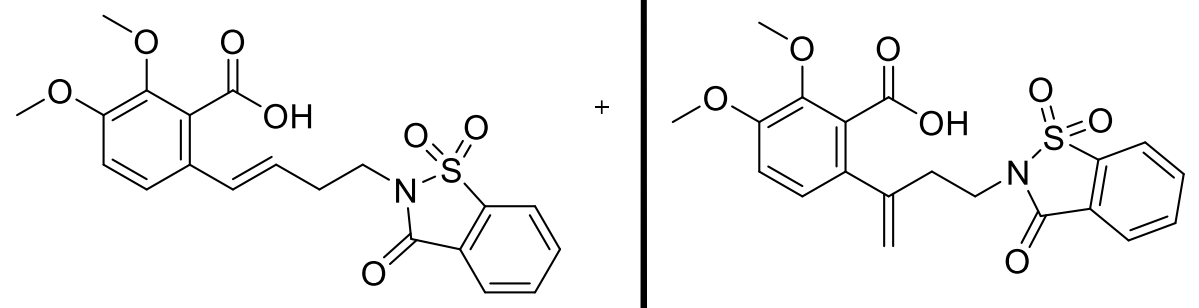

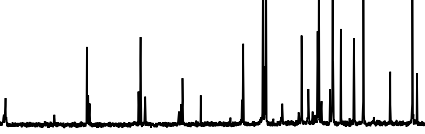

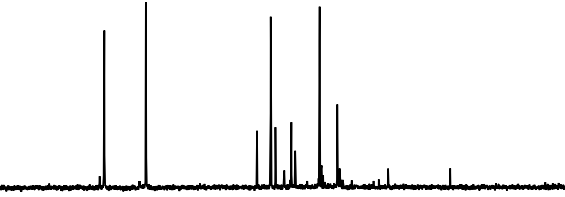

\begin{tabular}{|lllllllllllllllllllllllllllllll}
\hline 200 & 190 & 180 & 170 & 160 & 150 & 140 & 130 & 120 & 110 & $\begin{array}{c}100 \\
\mathrm{f1}(\mathrm{ppm})\end{array}$ & 90 & 80 & 70 & 60 & 50 & 40 & 30 & 20 & 10 & 0
\end{tabular} 
${ }^{1} \mathrm{H}$ and ${ }^{13} \mathrm{C}$ NMR Spectra of Compound 3al (DMSO solvent was used, $500 \mathrm{NMR} \mathrm{MHz}$ ).
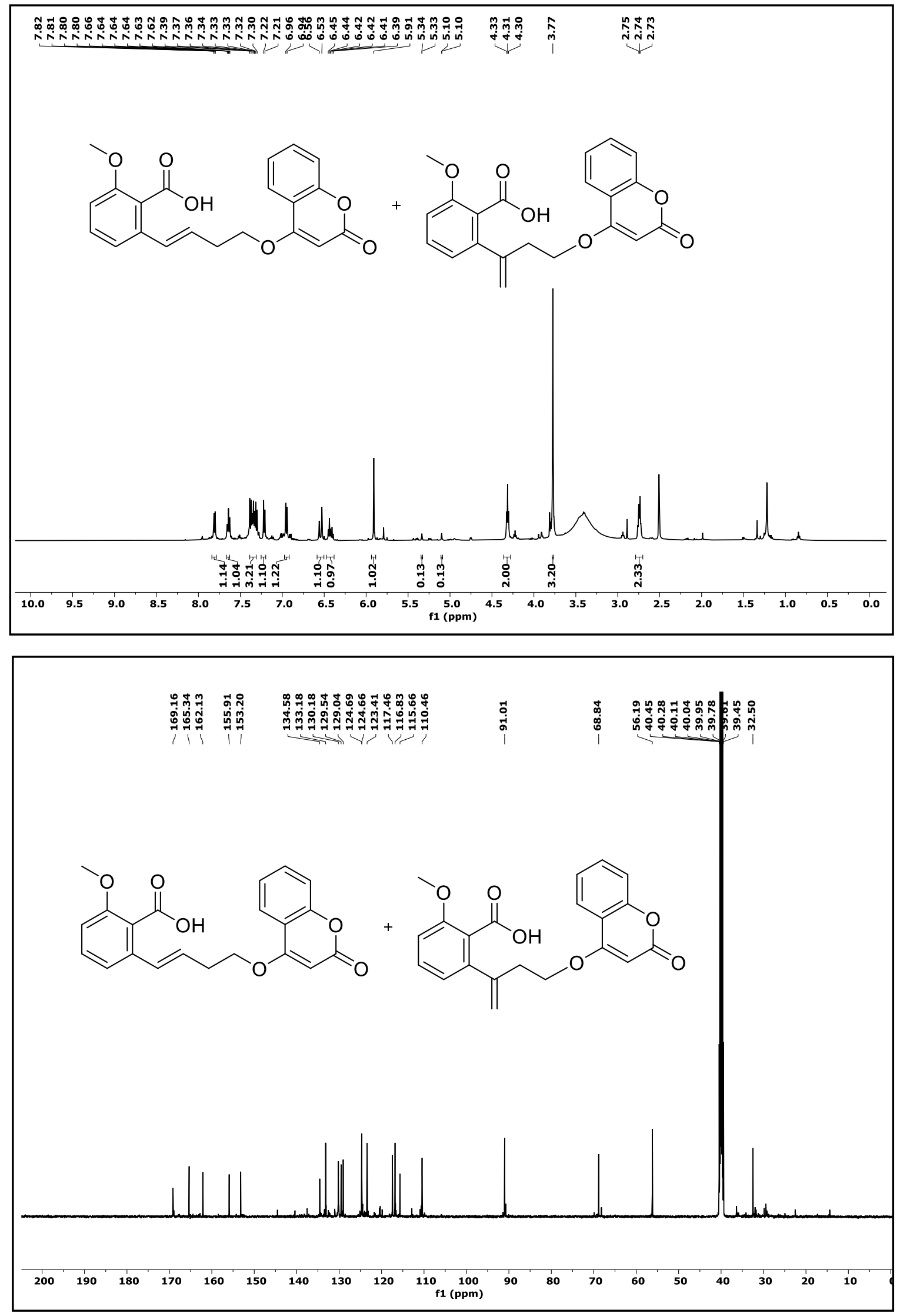
${ }^{1} \mathrm{H}$ and ${ }^{13} \mathrm{C}$ NMR Spectra of Compound 3nm. $\left(\mathrm{CDCl}_{3}\right.$ solvent was used, $\left.500 \mathrm{NMR} \mathrm{MHz}\right)$

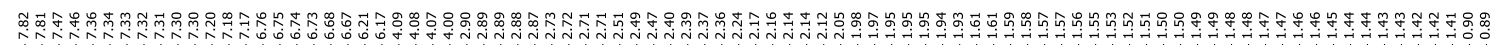

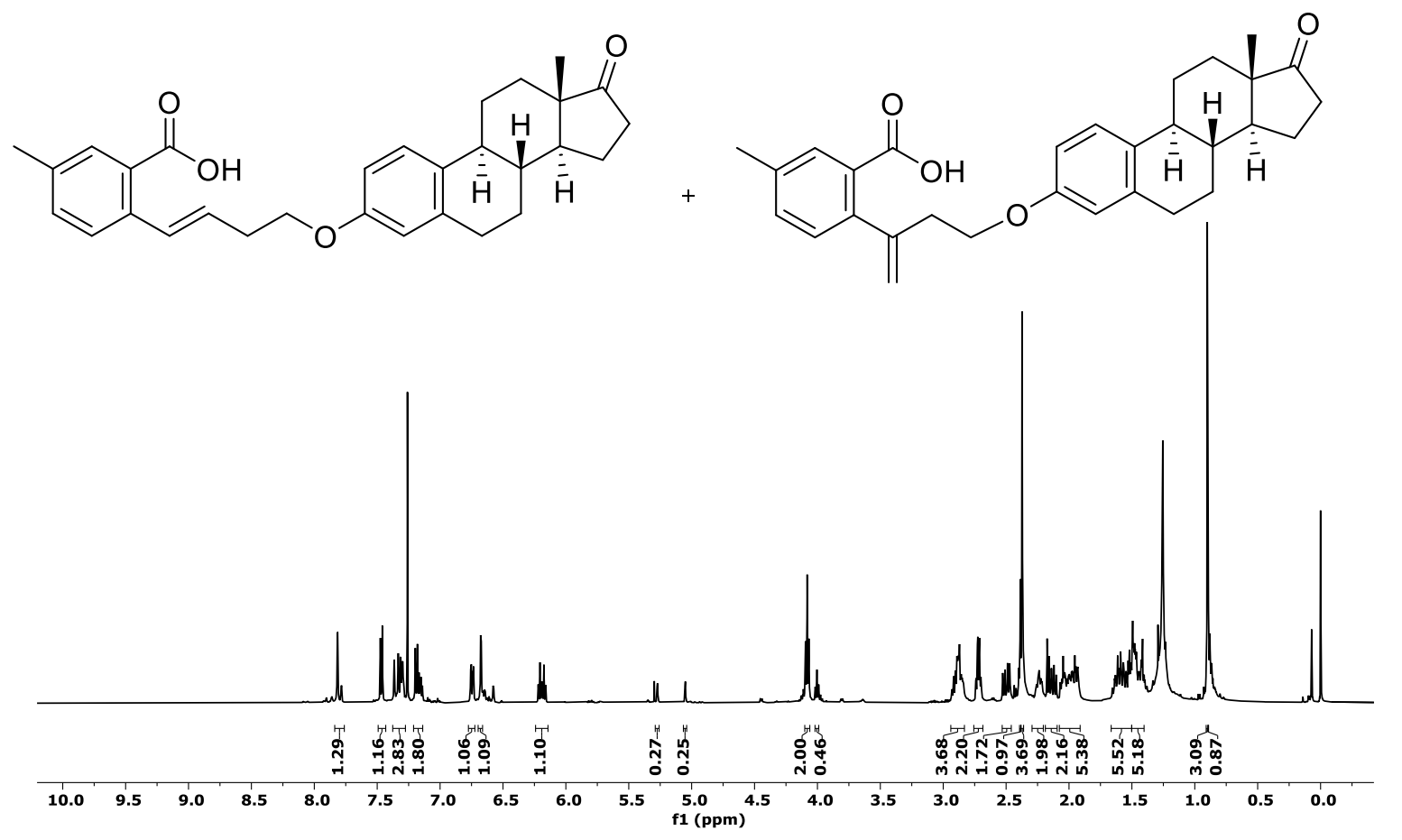

นดำ

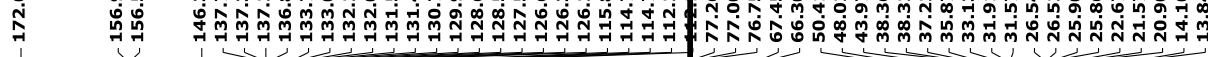

$C_{O}^{O C H}$

$\underbrace{O C O}_{1}$

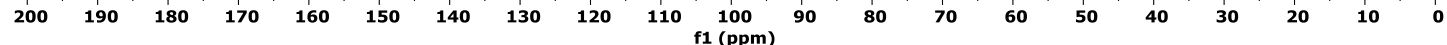


${ }^{1} \mathrm{H}$ and ${ }^{13} \mathrm{C}$ NMR Spectra of Compound 6aa. $\left(\mathrm{CDCl}_{3}\right.$ solvent was used, $\left.500 \mathrm{NMR} \mathrm{MHz}\right)$
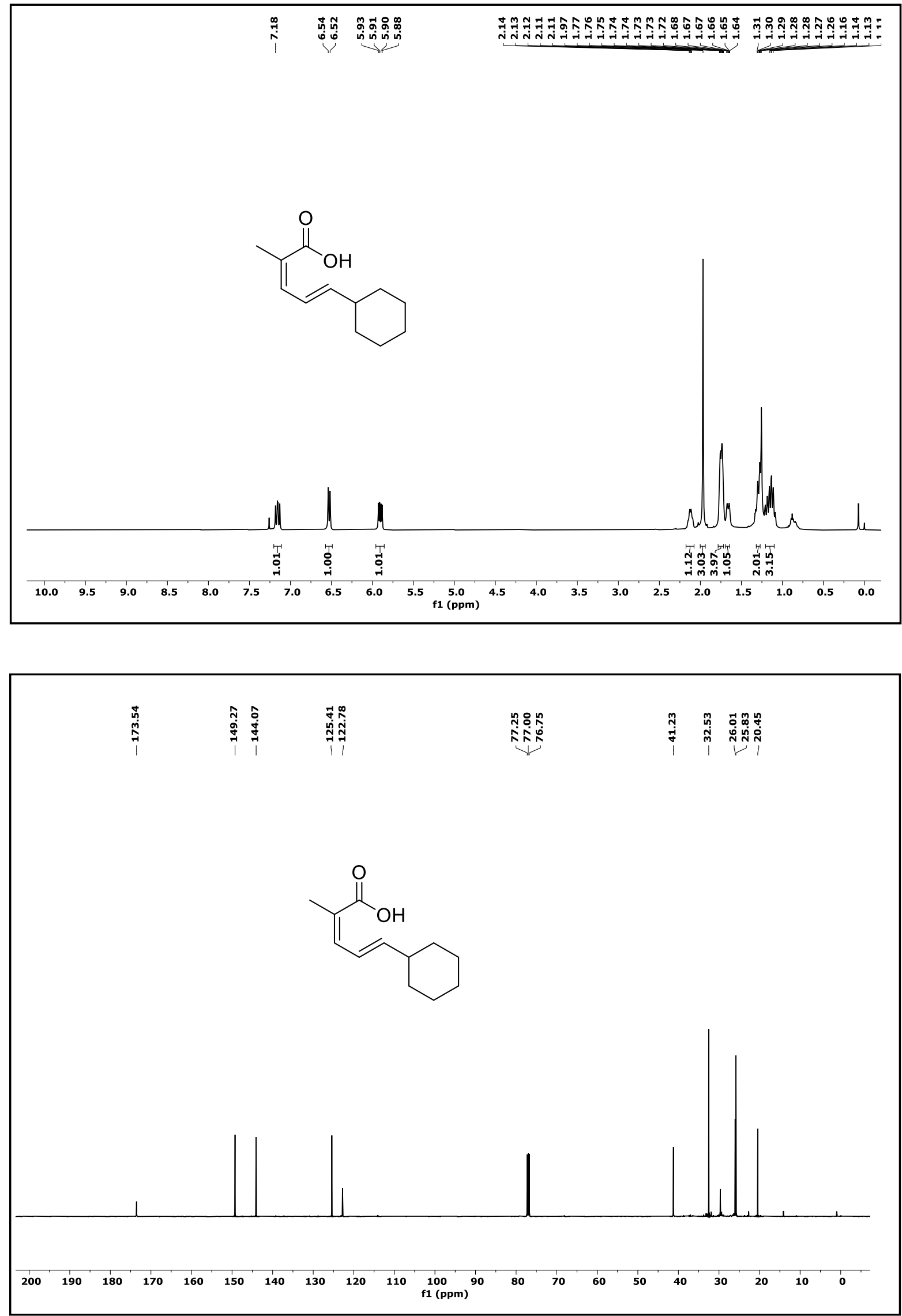
${ }^{1} \mathrm{H}$ and ${ }^{13} \mathrm{C}$ NMR Spectra of Compound 6ab. $\left(\mathrm{CDCl}_{3}\right.$ solvent was used, $\left.500 \mathrm{NMR} \mathrm{MHz}\right)$
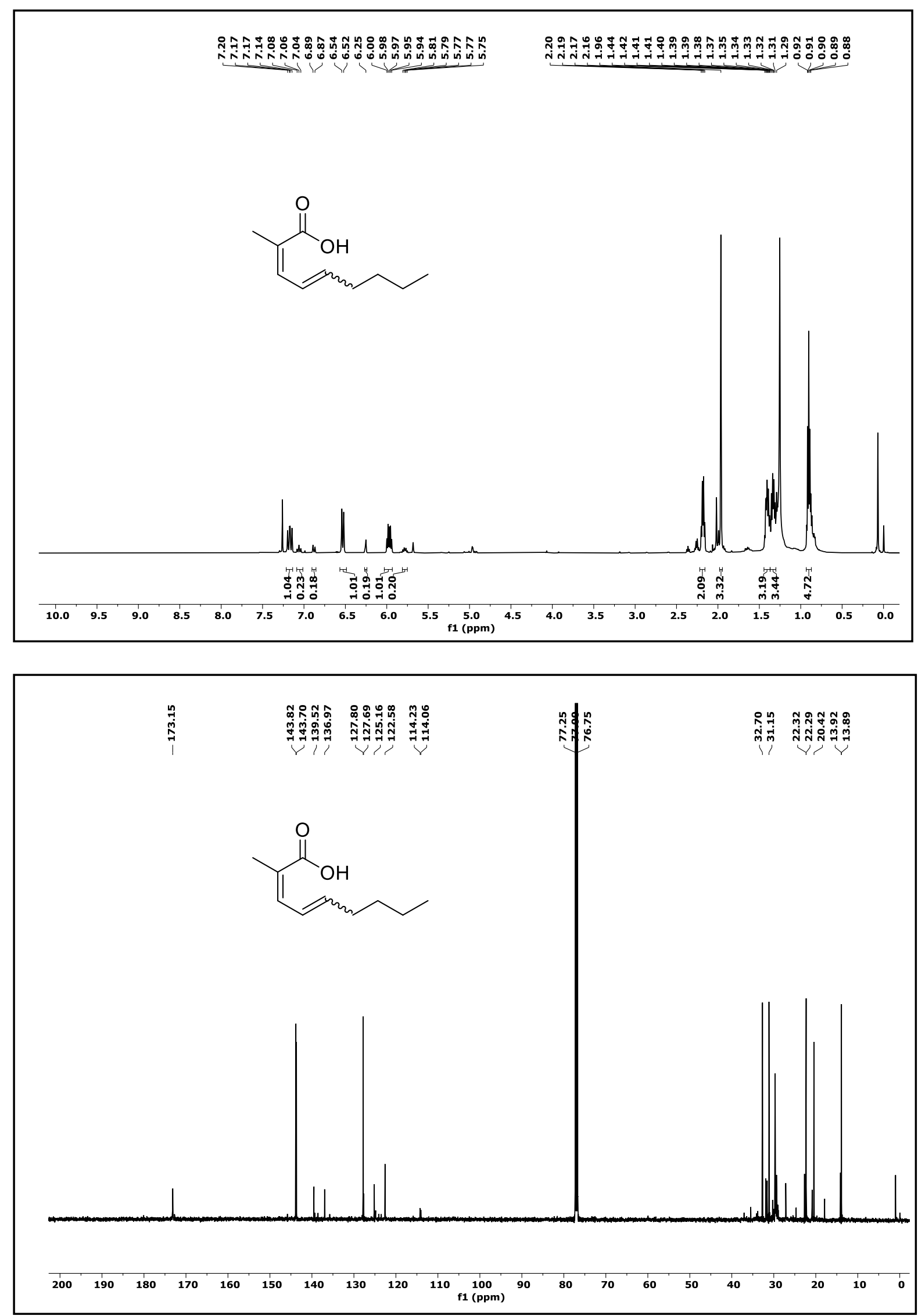
${ }^{1} \mathrm{H}$ and ${ }^{13} \mathrm{C}$ NMR Spectra of Compound 6ac. $\left(\mathrm{CDCl}_{3}\right.$ solvent was used, $\left.500 \mathrm{NMR} \mathrm{MHz}\right)$
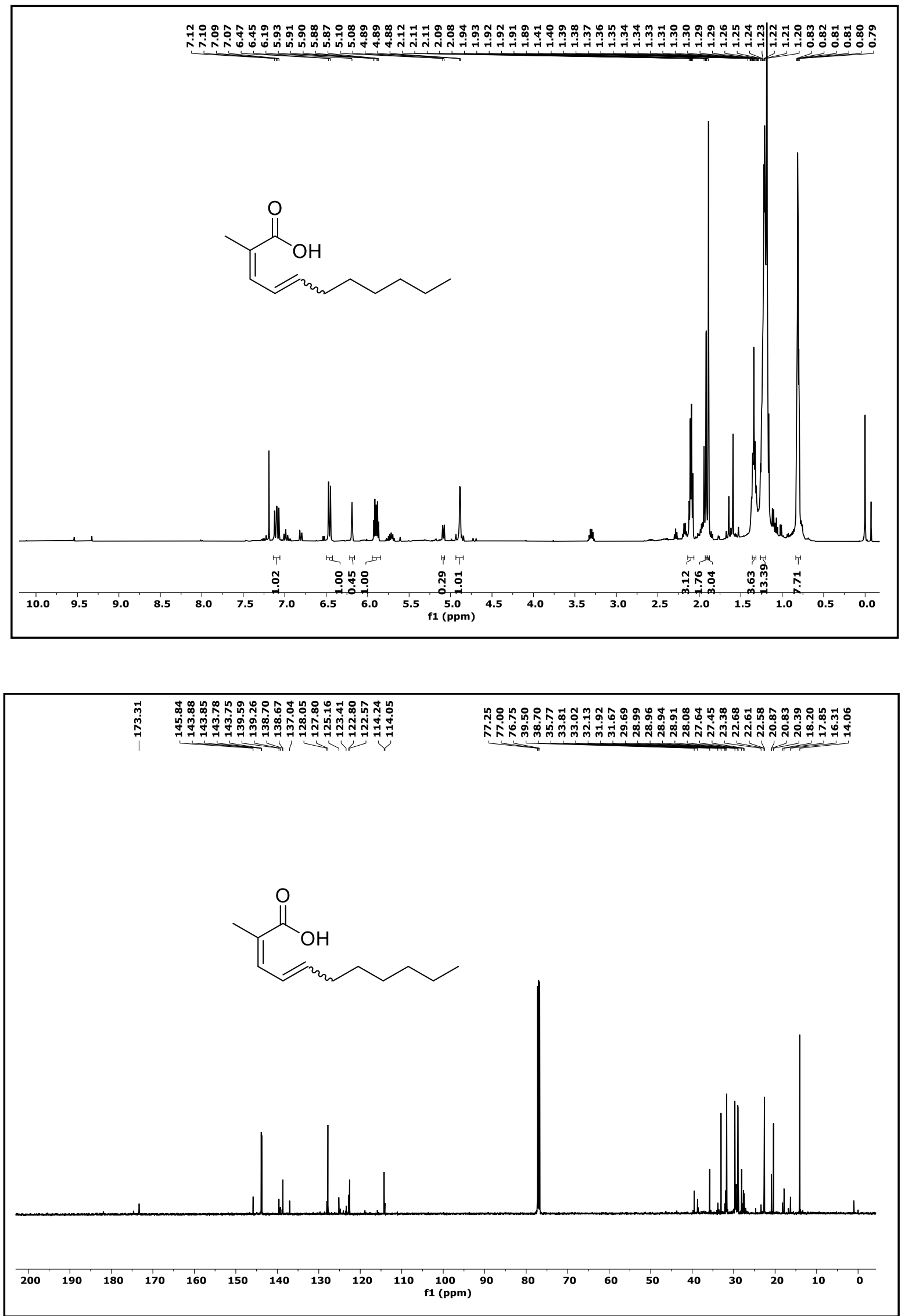
${ }^{1} \mathrm{H}$ and ${ }^{13} \mathrm{C}$ NMR Spectra of Compound 6ad. $\left(\mathrm{CDCl}_{3}\right.$ solvent was used, $\left.500 \mathrm{NMR} \mathrm{MHz}\right)$
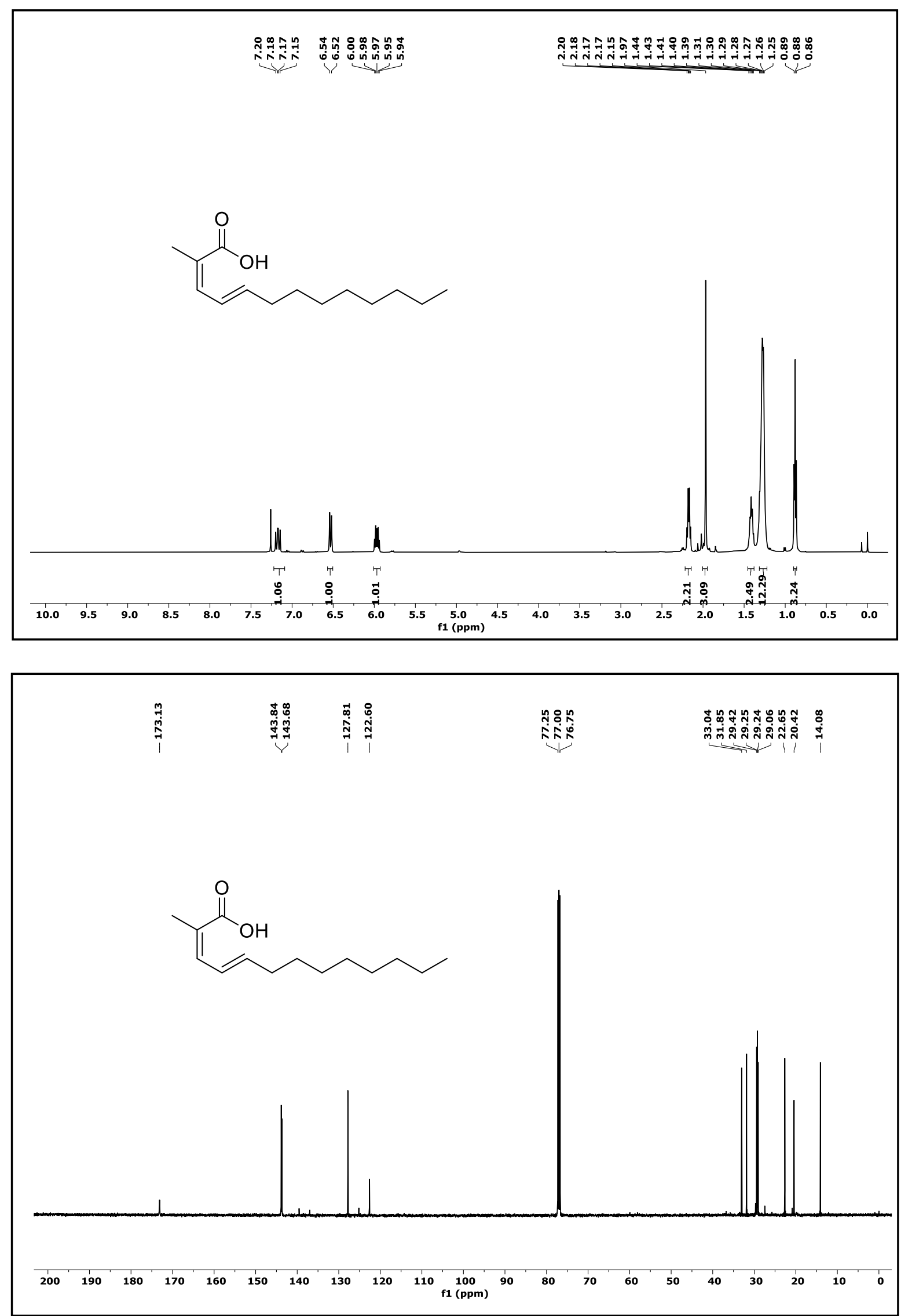
${ }^{1} \mathrm{H}$ and ${ }^{13} \mathrm{C}$ NMR Spectra of Compound 6af+6af'. $\left(\mathrm{CDCl}_{3}\right.$ solvent was used, $\left.500 \mathrm{NMR} \mathrm{MHz}\right)$
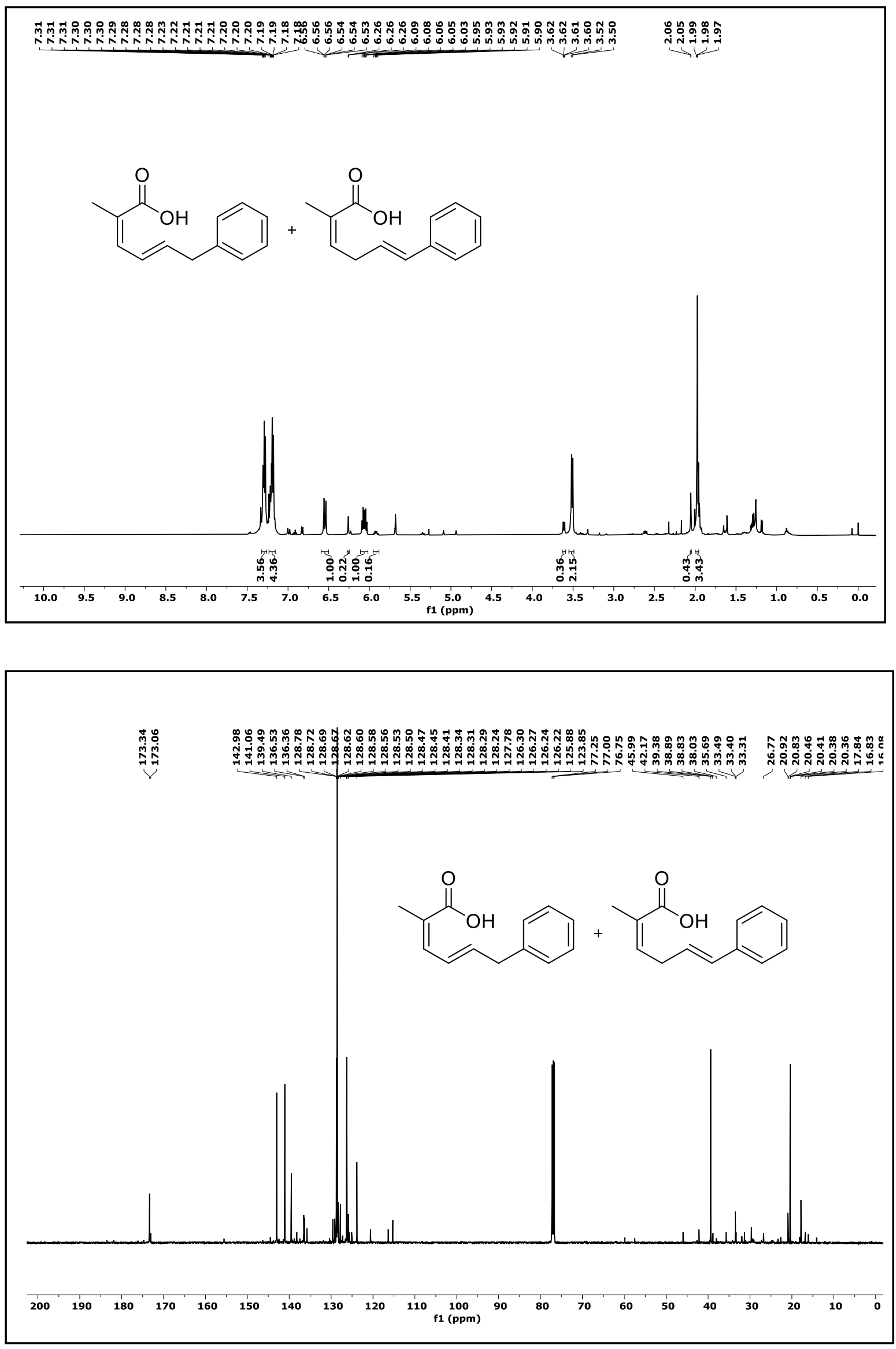
${ }^{1} \mathrm{H}$ and ${ }^{13} \mathrm{C}$ NMR Spectra of Compound 6ga. $\left(\mathrm{CDCl}_{3}\right.$ solvent was used, $\left.500 \mathrm{NMR} \mathrm{MHz}\right)$
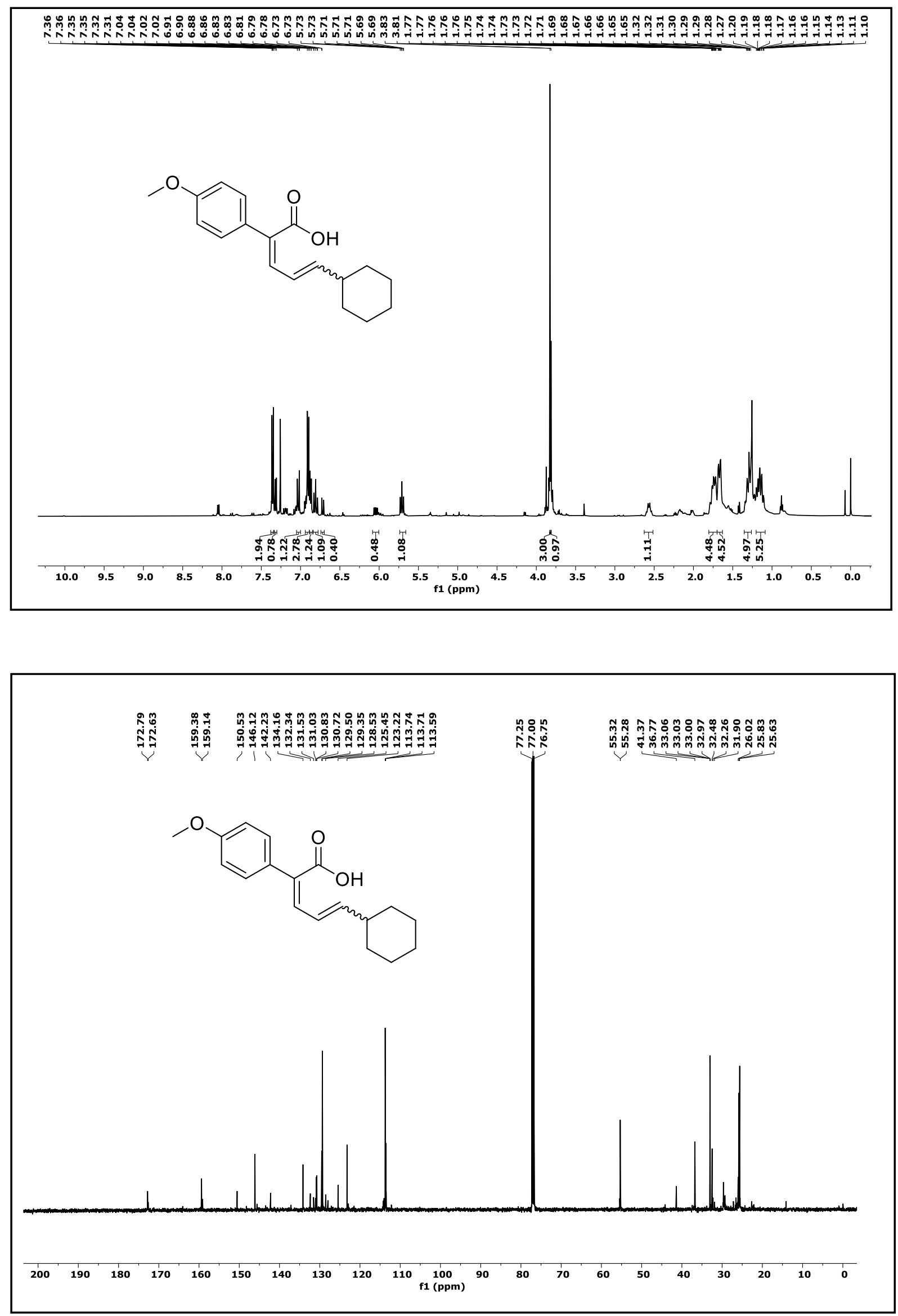